Florida International University

FIU Digital Commons

FIU Electronic Theses and Dissertations

University Graduate School

$3-26-2013$

\title{
Assessment of the Occurrence and Potential Risks of Pharmaceuticals and their Metabolites in Fish and Water Using Liquid Chromatography Mass Spectrometry
}

Jian Wang

Chemistry and Biochemistry, jwang010@fiu.edu

DOI: $10.25148 /$ etd.FI13042208

Follow this and additional works at: https://digitalcommons.fiu.edu/etd

\section{Recommended Citation}

Wang, Jian, "Assessment of the Occurrence and Potential Risks of Pharmaceuticals and their Metabolites in Fish and Water Using Liquid Chromatography Mass Spectrometry" (2013). FIU Electronic Theses and Dissertations. 835.

https://digitalcommons.fiu.edu/etd/835

This work is brought to you for free and open access by the University Graduate School at FIU Digital Commons. It has been accepted for inclusion in FIU Electronic Theses and Dissertations by an authorized administrator of FIU Digital Commons. For more information, please contact dcc@fiu.edu. 


\title{
FLORIDA INTERNATIONAL UNIVERSITY
}

Miami, Florida

\section{ASSESSMENT OF THE OCCURRENCE AND POTENTIAL RISKS OF PHARMACEUTICALS AND THEIR METABOLITES IN FISH AND WATER USING LIQUID CHROMATOGRAPHY MASS SPECTROMETRY}

\author{
A dissertation submitted in partial fulfillment of \\ the requirements for the degree of \\ DOCTOR OF PHILOSOPHY \\ in \\ CHEMISTRY \\ by \\ Jian Wang
}

2013 
To: Dean Kenneth G. Furton

College of Arts and Sciences

This dissertation, written by Jian Wang, and entitled Assessment of the Occurrence and Potential Risks of Pharmaceuticals and their Metabolites in Fish and Water Using Liquid Chromatography Mass Spectrometry, having been approved in respect to style and intellectual content, is referred to you for judgment.

We have read this dissertation and recommend that it be approved.

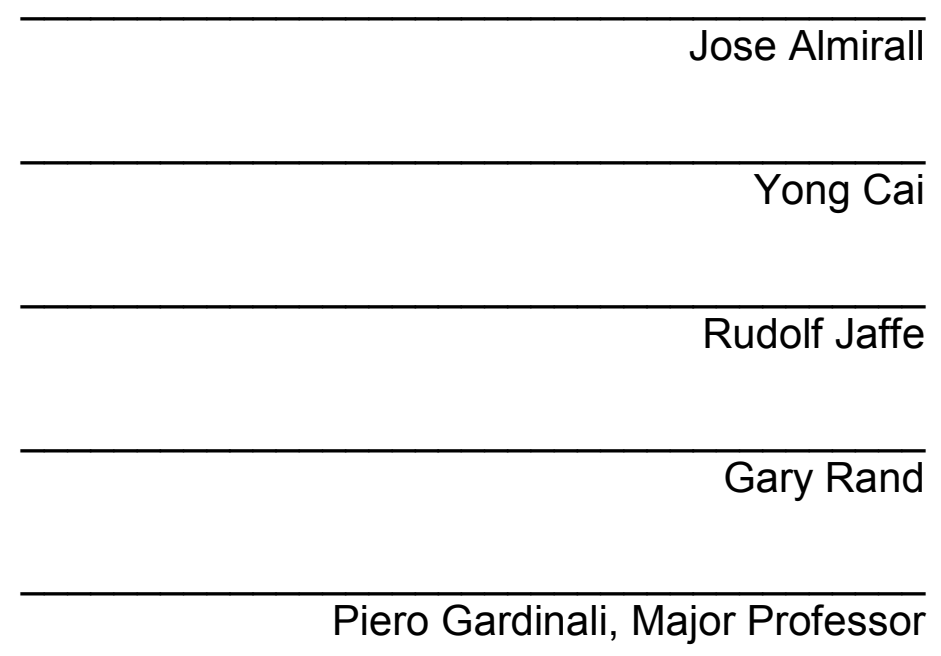

Date of Defense: March 26, 2013

The dissertation of Jian Wang is approved.

$\begin{array}{r}\text { Dean Kenneth G. Furton } \\ \text { College of Arts and Sciences } \\ \hline \begin{array}{c}\text { Dean Lakshmi N. Reddi } \\ \text { University Graduate School }\end{array}\end{array}$

Florida International University, 2013 


\section{DEDICATION}

To my father Yanzhong Wang and my mother Yuzhen Li, the greatest parents who are always there supporting me no matter what. 


\section{ACKNOWLEDGMENTS}

I would like to thank my major professor Dr. Piero Gardinali for being such an inspiring mentor along the road of pursuing my $\mathrm{PhD}$ in analytical chemistry. I enjoyed every second of the conversation between us from which I learned the importance of critical thinking and what it took to become a better scientist. I would also like to thank my committee members Dr. Rudolf Jaffe, Dr. Yong Cai, Dr. Jose Almirall and Dr. Gary Rand for sharing their insightful ideas and providing helpful comments to my research.

I would like to thank Natalia for being such a good friend who is always ready to help me out and talk to. Special thanks go to Ingrid and Adolfo for putting the efforts in maintaining the laboratory like a home. I would also like to thank Patricia, Cesar, Chengtao, Nubia, Mark, Kathia, Sudha, Venkat, Eli and all the co-workers and lab mates for leaving me such a memorable time studying in FIU. A special thank goes to my girlfriend, Yan Ding. I could have never achieved what I have today without you. 


\section{ABSTRACT OF THE DISSERTATION \\ ASSESSMENT OF THE OCCURRENCE AND POTENTIAL RISKS OF \\ PHARMACEUTICALS AND THEIR METABOLITES IN FISH AND WATER \\ USING LIQUID CHROMATOGRAPHY MASS SPECTROMETRY}

by

Jian Wang

Florida International University, 2013

Miami, Florida

Professor Piero Gardinali, Major Professor

A comprehensive method for the analysis of 11 target pharmaceuticals representing multiple therapeutic classes was developed for biological tissues (fish) and water. Water samples were extracted using solid phase extraction (SPE), while fish tissue homogenates were extracted using accelerated solvent extraction (ASE) followed by mixed-mode cation exchange SPE cleanup and analyzed by liquid chromatography tandem mass spectrometry (LC-MS/MS). Among the 11 target pharmaceuticals analyzed, trimethoprim, caffeine, sulfamethoxazole, diphenhydramine, diltiazem, carbamazepine, erythromycin and fluoxetine were consistently detected in reclaimed water. On the other hand, caffeine, diphenhydramine and carbamazepine were consistently detected in fish and surface water samples.

In order to understand the uptake and depuration of pharmaceuticals as well as bioconcentration factors (BCFs) under the worst-case conditions, mosquito fish were exposed to reclaimed water under static-renewal for 7 days, followed by a 
14-day depuration phase in clean water. Characterization of the exposure media revealed the presence of 26 pharmaceuticals while 5 pharmaceuticals including caffeine, diphenhydramine, diltiazem, carbamazepine, and ibuprofen were present in the organisms as early as $5 \mathrm{~h}$ from the start of the exposure.

Liquid chromatography ultra-high resolution Orbitrap mass spectrometry was explored as a tool to identify and quantify phase II pharmaceutical metabolites in reclaimed water. The resulting data confirmed the presence of acetylsulfamethoxazole and sulfamethoxazole glucuronide in reclaimed water. To my knowledge, this is the first known report of sulfamethoxazole glucuronide surviving intact through wastewater treatment plants and occurring in environmental water samples.

Finally, five bioaccumulative pharmaceuticals including caffeine, carbamazepine, diltiazem, diphenhydramine and ibuprofen detected in reclaimed water were investigated regarding the acute and chronic risks to aquatic organisms. The results indicated a low potential risk of carbamazepine even under the worst case exposure scenario. Given the dilution factors that affect environmental releases, the risk of exposure to carbamazepine will be even more reduced. 


\section{TABLE OF CONTENTS}

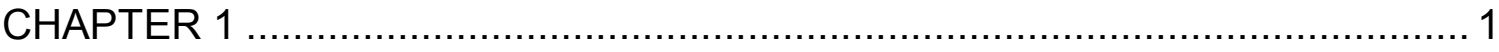

1.1 Occurrence of pharmaceutically active compounds in the environment......2

1.2 Adverse effects under chronic exposure ................................................ 3

1.3 Reclaimed water exposure ............................................................... 4

1.4 Current analytical protocols of detecting pharmaceuticals in water and biological samples .............................................................................. 4

1.5 Why is it needed to improve the throughput of current on-line SPE

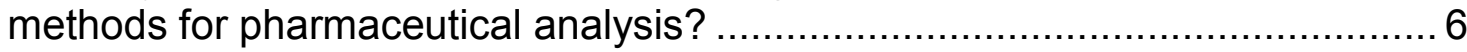

1.6 Why is pharmacokinetics under the "worst case scenario" important? ........ 8

1.7 Identification of pharmaceutical metabolites in reclaimed water ................. 9

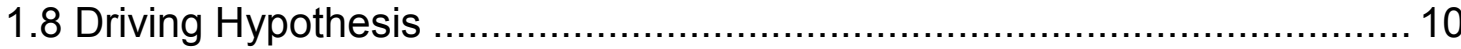

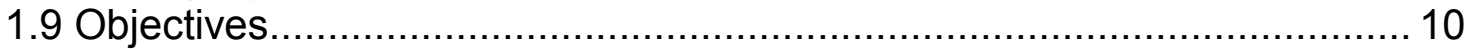

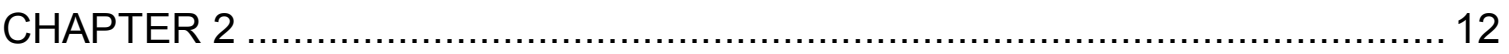

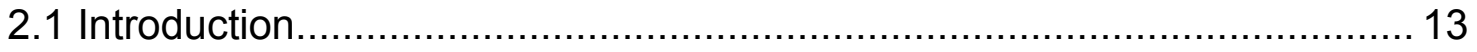

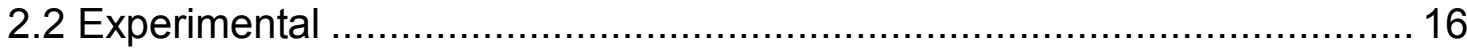

2.2.1 Reagents, standards and solutions ............................................. 16

2.2.2 Sample collection and storage .................................................. 17

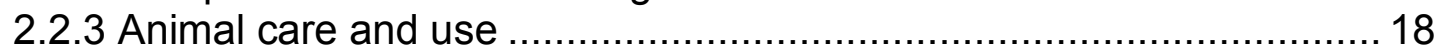

2.2.4 Solid phase extraction for water samples.................................... 18

2.2.5 ASE extraction for fish tissues .............................................. 19

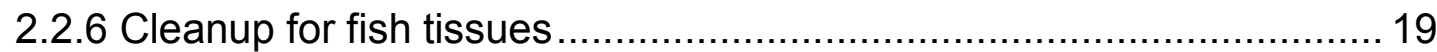

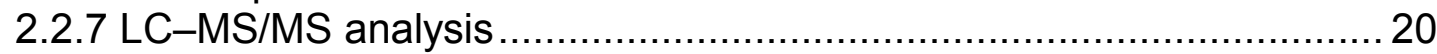

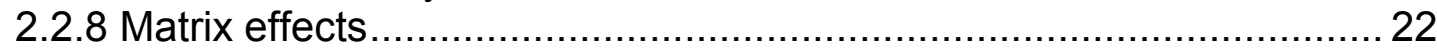

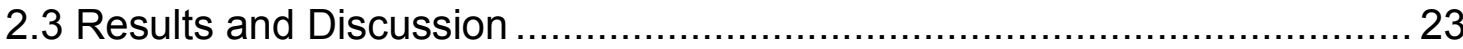

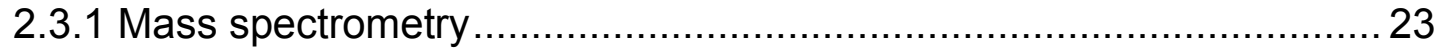

2.3.2 Accelerated solvent extraction for fish tissues ............................... 25

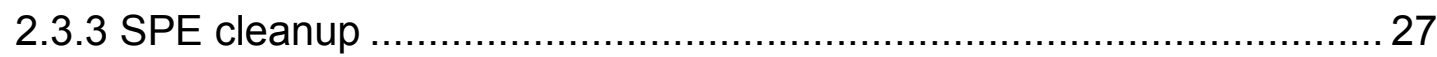

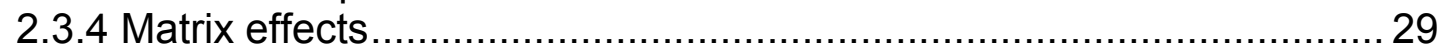

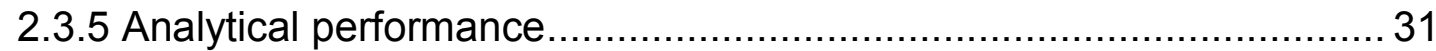

2.3.6 Analysis of environmental samples .............................................. 35

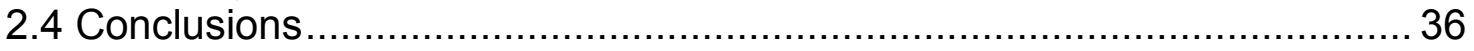

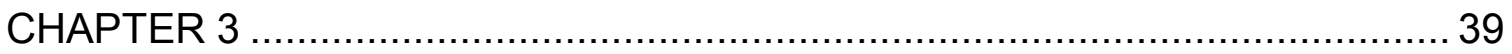

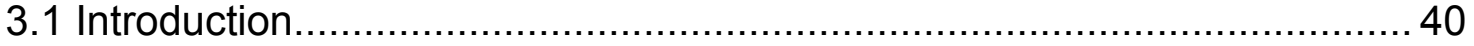

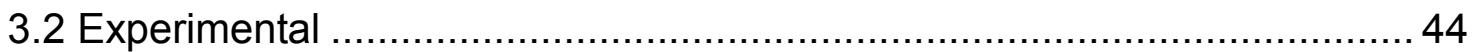

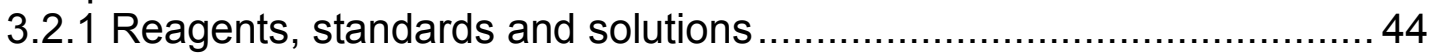

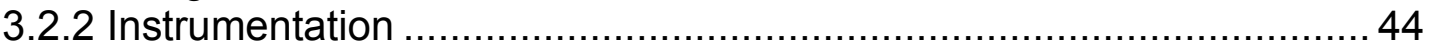

3.2.3 Working principle of EQuan system .............................................. 45

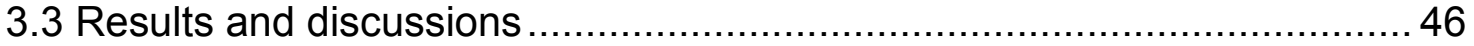




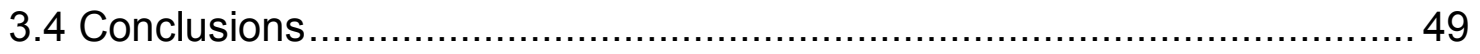

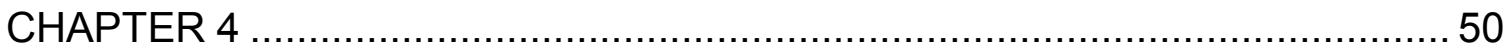

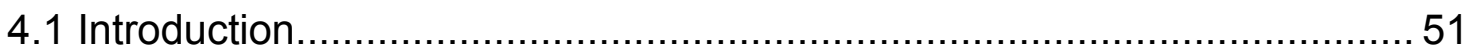

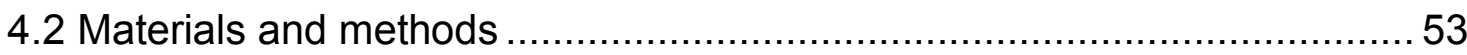

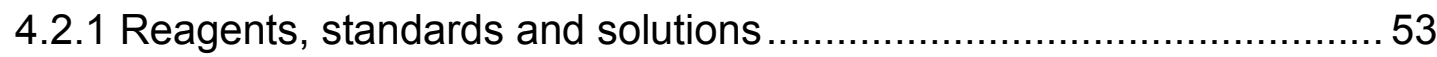

4.2.2 Organism husbandry and exposure conditions ................................5 54

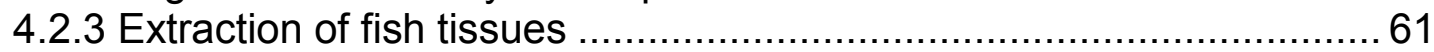

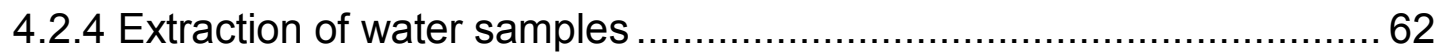

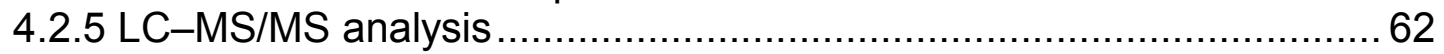

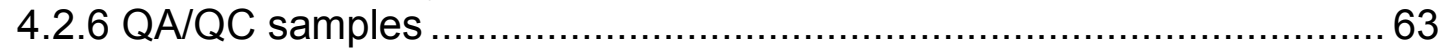

4.2.7 Pharmacokinetics and bioconcentration analysis............................. 64

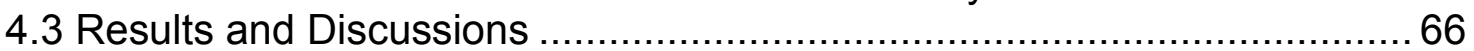

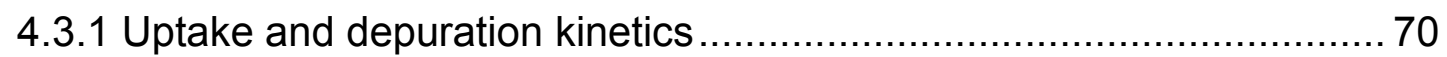

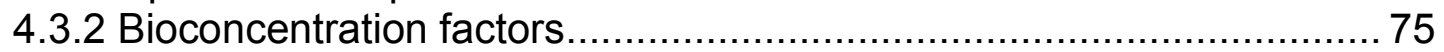

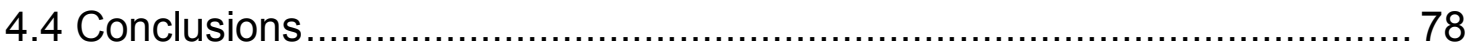

CHAPTER 5

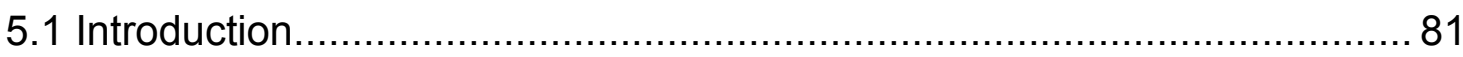

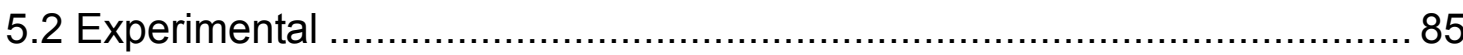

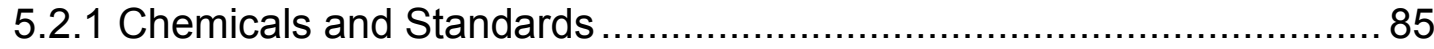

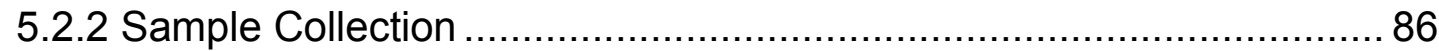

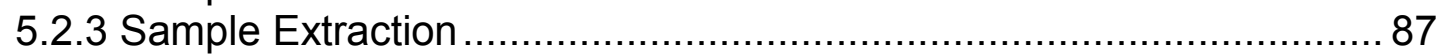

5.2.4 Liquid Chromatography and Q Exactive Mass spectrometry ............. 87

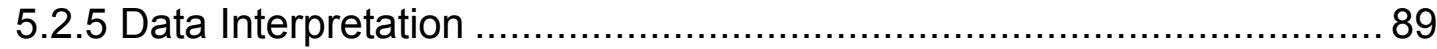

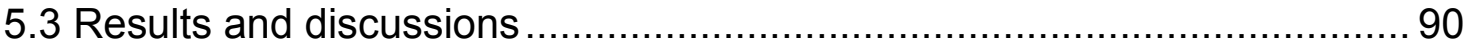

5.3.1 Performance of Q Exactive Orbitrap .............................................. 90

5.3.2 Tentative Identification of Phase II metabolites based on Accurate

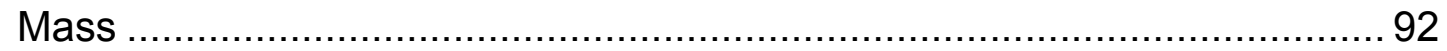

5.3.3 Identification of Acetyl-sulfamethoxazole and Sulfamethoxazole

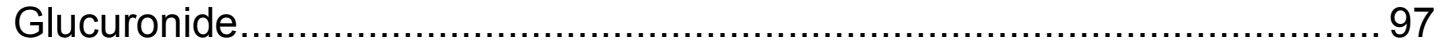
5.3.4 Determination of Sulfamethoxazole and Its Metabolites in Reclaimed

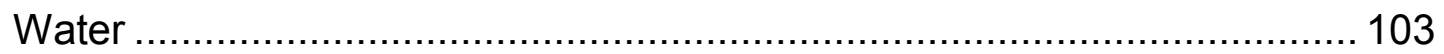
5.3.5 Occurrence of Sulfamethoxazole and Its Metabolites in Reclaimed

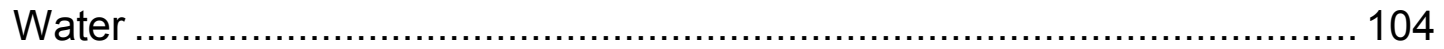

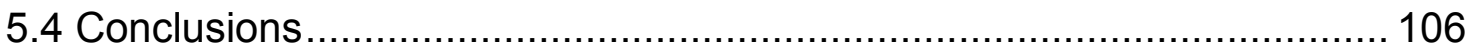

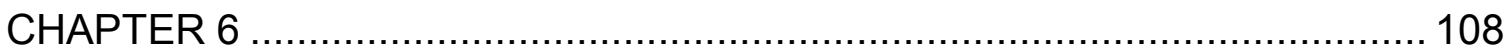

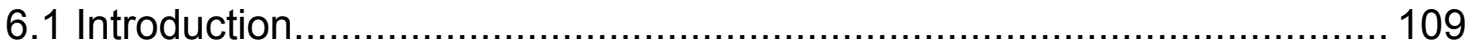

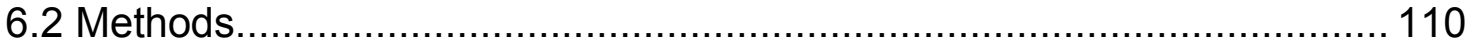

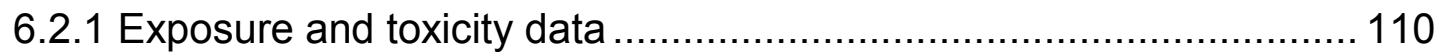

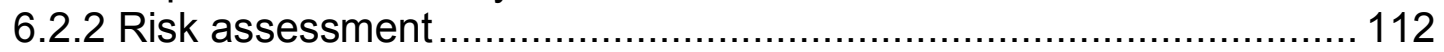

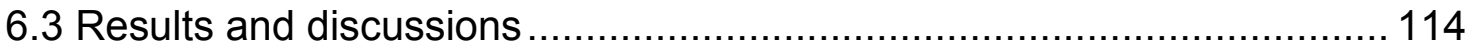

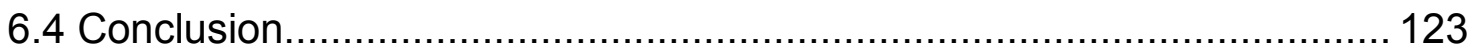


CONCLUSIONS

REFERENCES.

VITA 


\section{LIST OF TABLES}

\section{TABLE}

PAGE

Table 2.1 SRM transition parameters

Table 2.2 Observed MDLs Matrix Effects and recoveries in fish tissue 30

Table 2.3 Observed MDLs matrix effects and recoveries in pond water and reclaimed water

Table 2.4 Fortification levels for matrix effect assessment, recovery calculation and MDL determination in fish tissues and water samples

Table 2.5 Summary of concentrations of target compounds in reclaimed water and pond water

Table 2.6 Concentrations $(\mathrm{ng} / \mathrm{g})$ of target compounds detected in fish tissue $(n=7)$ from the freshwater pond

Table 4.1 Identity, source and physicochemical parameters (Log $\left.K_{\text {ow }}\right)$ for all compounds used in the study

Table 4.2 Pharmaceuticals detected in exposure reclaimed water. 67

Table 4.3 Uptake and depuration rate constants, half-lives and BCFs for selected pharmaceuticals in mosquito fish (Gambusia holbrooki)

Table 5.1 Elemental compositions, exact masses, observed accurate masses, and mass accuracy of selected parent pharmaceuticals

Table 5.2 Monitored phase II modifications and exact masses of the modifications to parent pharmaceuticals

Table 6.1 90th and 50th centiles of exposure concentrations

Table 6.2 The 5th and 10th centiles of toxicity data from acute and chronic SSDs

Table 6.3 Statistics and potentially affected fraction (PAF) for acute and chronic toxicity data for pharmaceuticals 


\section{LIST OF FIGURES}

FIGURE

PAGE

Figure 2.1 Representative chromatograms of all selected pharmaceuticals standards on HESI (A) and ESI (B) sources at the same spiking level...... 25

Figure 2.2 ASE solvent selection based on absolute recoveries 27

Figure 2.3 Averaged $(n=3)$ individual analyte recoveries in each wash solvent system

Figure 3.1 Comparison of run times among a typical program, look ahead and the modified program for a $20 \mathrm{~mL}$ on-line EQuan injection with $10 \mathrm{~min}$ separation time.

Figure 3.2 Valve configurations and CTC autosampler commands in a typical autosampler program and the new autosampler program ....

Figure 3.3 Scheme of on-line SPE EQuan system based on columnswitching

Figure 4.1 Uptake and depuration curves for mosquito fish (Gambusia holbrooki) exposed to reclaimed water: the dotted lines represent for the Michaelis-Menten curve fit and the solid lines represent for the first order curve fit

Figure 4.2 Correlations between bioconcentration factors and $\log K_{\mathrm{ow}}\left(\mathrm{BCF}_{\mathrm{a}}\right.$ : based on steady state concentrations, $\mathrm{BCF}_{\mathrm{b}}$ : based on rate constants, and $\mathrm{BCF}_{\mathrm{c}}$ : based on saturation state concentrations)...

Figure 5.1 LC-Q Exactive Orbitrap extracted ion chromatograms (XICs) of sulfamethoxazole ( $\mathrm{m} / \mathrm{z} 254.0594)$, sulfamethoxazole glucuronide $(\mathrm{m} / \mathrm{z}$ $430.0915)$ and acetyl-sulfamethoxazole $(\mathrm{m} / \mathrm{z} 296.0700)$ in a reclaimed water sample; mass tolerance was set to $5 \mathrm{ppm}$ for all compounds

Figure 5.2 High resolution data-dependent MS/MS spectra and major fragments for A: sulfamethoxazole standard, B: sulfamethoxazole in reclaimed water, C: acetyl-sulfamethoxazole in reclaimed water, and D:

sulfamethoxazole glucuronide in reclaimed water 101

Figure 5.3 Proposed fragmentation pathways for A: sulfamethoxazole, B: acetyl-sulfamethoxazole, and C: sulfamethoxazole glucuronide 103 
Figure 5.4 Occurrence of sulfamethoxazole and its two phase II metabolites acetyl-sulfamethoxazole and sulfamethoxazole glucuronide in reclaimed water during a period of one month $(n=7)$

Figure 6.1 Boxplot of exposure concentrations $(n=7)$ of selected pharmaceuticals in reclaimed water

Figure 6.2 Acute species sensitivity distributions with 95\% Confidence Interval for caffeine, carbamazepine, diltiazem, diphenhydramine and ibuprofen

Figure 6.3 Chronic species sensitivity distributions with 95\% Confidence Interval for caffeine, carbamazepine, diphenhydramine and ibuprofen

Figure 6.4 Joint probability curve for the exposure concentration and chronic toxicity data of carbamazepine 
ABBREVIATION

ASE

BAF

$\mathrm{BCF}$

EPA

ESI

GC

HLB

HPLC

LC-MS

LIT

MDLs

MS

PPCPs

QqQ

$\mathrm{RRF}$

$\mathrm{S} / \mathrm{N}$

SPME

SPE

SIM

SRM

TOF
FULLNAME

accelerated solvent extraction

bioaccumulation factor

bioconcentration factor

Environmental Protection Agency

electrospray ionization

gas chromatography

Hydrophilic-Lipophilic Balanced

high performance liquid chromatography liquid chromatography-mass spectrometry

linear ion trap

method detection limits mass spectrometry pharmaceuticals and personal care products triple quadrupole relative response factor signal to noise ratio solid phase microextraction solid phase extraction selected ion monitoring selected reaction monitoring time of flight 
WWTPs

MCX

XIC

ERA

PAF

JPC wastewater treatment plants mixed mode cation exchange extracted ion chromatogram ecological risk assessment potentially affected fraction joint probability curve 
CHAPTER 1

Introduction 


\subsection{Occurrence of pharmaceutically active compounds in the environment}

Pharmaceutically active compounds are now well-acknowledged environmental pollutants. They are released into the environment largely through the discharge of wastewater treatment plants (WWTPs) as a result of excretion and metabolism by humans and animals, and additionally, disposal of unused or expired drugs (Daughton and Ternes 1999; Miao and Metcalfe 2003; Vanderford et al. 2003). However, most WWTPs are not specifically designed to remove most of the pharmaceuticals, thus these compounds are present in reclaimed and surface waters in a wide range of concentrations (Heberer 2002; Boyd et al. 2003).

Besides parent pharmaceuticals, the presence of pharmaceutically active compounds in the environment can also be in the form of transformation products (i.e. 10,11-dihydro-10,11-dihydroxycarbamazepine, the major metabolite of carbamazepine, and norfluoxetine, the major metabolite of fluoxetine) (Miao and Metcalfe 2003; Brooks et al. 2005; Miao et al. 2005; Chu and Metcalfe 2007). Parent drugs can be excreted as the unchanged form (Miao et al. 2005) whereas transformation products can be formed as metabolites by undergoing chemical or biochemical transformation (Gobel et al. 2004) or as photodegradation products by exposure to sunlight (Bonvin et al. 2012). Current research is heavily focusing on unchanged parent drugs (Celiz et al. 2009). However, it is important to realize that the exposure to metabolites may have hazardous effects similar to those of the parent drugs (Bedner and MacCrehan 2006). 


\subsection{Adverse effects under chronic exposure}

Although concentrations in environmental water bodies are typically at $\mathrm{ng} / \mathrm{L}$ to low $\mu \mathrm{g} / \mathrm{L}$ level, these organic pollutants may still pose risks to aquatic species under chronic long-term exposure (Vanderford et al. 2003; Brooks et al. 2005;

Owen et al. 2007; Gunnarsson et al. 2008). For instance, after a 21-day exposure, fluoxetine was shown to induce vitellogenin in male fathead minnows (Pimephales promelas) at $28 \mathrm{ng} / \mathrm{L}$ and venlafaxine caused mortality of fathead minnows at concentration as low as $305 \mathrm{ng} / \mathrm{L}$ (Schultz et al. 2011). Similarly, plasma samples from the rainbow trout (Oncorhynchus mykiss) exposed to sewage effluents were analyzed for 25 pharmaceuticals, of which levonorgestrel was detected in fish plasma at concentrations $(8.5-12 \mathrm{ng} / \mathrm{mL})$ exceeding the human therapeutic plasma level (Fick et al. 2010). Because many aquatic species were shown to have similar physiological receptors to those the pharmaceuticals are originally intended to react with in humans (Gunnarsson et al. 2008; Quinn et al. 2009), the data suggested a high risk of pharmacological effects on rainbow trout (Fick et al. 2010).

Another study concerning the uptake and depuration as well as bioconcentration factors (BCFs) of pharmaceuticals including moclobemide, 5-fluoruracil, carbamazepine, diazepam, carvedilol and fluoxetine was conducted in freshwater shrimp (Gammarus pulex) and water boatman (Notonecta glauca) after a 2-day uptake phase (Meredith-Williams et al. 2012). The results showed that BCFs in freshwater shrimp were significantly higher than those found in water boatman, 
implying that pharmaceuticals may possess even higher risks to smaller aquatic species as environmental pollutants.

\subsection{Reclaimed water exposure}

A freshwater pond located at Florida International University Biscayne Bay Campus (North Miami Beach, FL) continuously receives storm water runoff and reclaimed water from a local WWTP through daily sprinkler irrigation. The source of reclaimed water was Miami-Dade Water and Sewer Department North District Wastewater Treatment Plant. Pure oxygen activated-sludge was used in the WWTP as the main secondary treatment process. Extra filtration and disinfection were applied to effluents before release to make the reclaimed water ready for use in irrigation. Fish are sensitive indicators for the substances that enter aquatic ecosystems (EPA 2009). Therefore, the aquatic organisms living in this pond are likely to be chronically exposed to the contaminants from reclaimed water. It is also expected that aquatic systems like this freshwater pond influenced by substantial inputs of reclaimed water may represent a good model to study the exposure of biological resources to mixtures of pharmaceuticals under relatively natural conditions (Brooks et al. 2006).

\subsection{Current analytical protocols of detecting pharmaceuticals in water and biological samples}

On the basis of the complexity of environment matrices and the multiple functionalities of emerging contaminants as target compounds, recently 
described analytical protocols for the determination of pharmaceuticals in both wastewaters (Segura 2007; Lajeunesse et al. 2008; Segura 2009) and biological organisms (Tavazzi et al. 2002; Brooks et al. 2005; Chu and Metcalfe 2007; Berrada et al. 2008) have focused on rather specific classes of compounds. Antidepressants (Brooks et al. 2005; Chu and Metcalfe 2007; Lajeunesse et al. 2008) and antibiotics (Segura 2007; Berrada et al. 2008; Segura 2009) are the two classes that have been studied the most. As the list of compounds found in field collected fish samples expands, the need to develop simultaneous screening methods for multiple classes of drug residues increases as well (Balmer et al. 2004; Duedahl-Olesen et al. 2005; Buser et al. 2006; Rudel et al. 2006; Mottaleb et al. 2009). At present, the general approach employed for the analysis of multi-class pharmaceuticals in fish involves extraction of homogenized tissue with 1:1 mixture of $0.1 \mathrm{M}$ acetic acid and methanol (Ramirez et al. 2007), or acetonitrile combined with limited cleanup to back-extract lipid material followed by liquid chromatography-mass spectrometry (LC-MS) analysis (Smith et al. 2009). Additionally, the use of solid-phase microextraction (SPME) techniques has also been explored as surrogates to estimate the potential occurrence and uptake of pharmaceuticals in living fish by assuming similar partition behavior between the SPME devices and the fish tissues placed in contaminated environments (Zhou et al. 2008).

Accelerated solvent extraction (ASE) involving high pressure and temperature has been shown to efficiently extract a wide range of compounds from fish 
tissues (Draisci et al. 1998; Datta et al. 2002; Tavazzi et al. 2002; Wahlen 2004; Chu and Metcalfe 2007; Haglund et al. 2007; Berrada et al. 2008; Llorca et al. 2009; Losada et al. 2009; Lund et al. 2009). Meanwhile, HLB polymeric cartridges are routinely used as SPE sorbent for cleanup of complex environmental samples prior to LC-MS analysis. However, on the basis of considerable variation in lipophilicity and $\mathrm{pKa}$ among different classes of pharmaceuticals, optimum cleanup efficiency can be compromised by differential retention behavior of target analytes on the sorbent. In contrast, mixed-mode cation exchange (MCX) cartridges can accommodate both neutrals and cations providing better selectivity during elution steps. For instance, Chu et al. (Chu and Metcalfe 2007) developed a method to determine paroxetine, fluoxetine and its metabolite in fish tissue using accelerated solvent extraction followed by MCX cleanup offering better recoveries and minimized matrix interferences. Because of the capabilities mentioned above, mixed-mode MCX cartridges will likely provide the needed retention for the multiple classes of pharmaceuticals proposed herein.

\subsection{Why is it needed to improve the throughput of current on-line SPE methods for pharmaceutical analysis?}

As the list of pharmaceutically active compounds being reported in various environmental matrices keeps growing, it is of obvious importance to develop new analytical techniques that are more effective at analyzing multiple components in a large number of samples in limited time (Balmer et al. 2004; 
Buser et al. 2006; Mottaleb et al. 2009). In order to address the needs for lower detection limits and higher sample throughput, an on-line SPE coupled to mass spectrometry detection was developed to eliminate several preparation steps such as evaporation and reconstitution (Segura 2007; Garcia et al. 2009; Wang and Gardinali 2012). More recently, on-line SPE LC-MS/MS has become a more desired technique in routine analysis of aqueous samples, especially for the samples that are time sensitive and need large injection volume to improve sensitivity.

The time for a typical on-line SPE LC-MS/MS analysis consists of autosampler preparation time, sample withdraw time, SPE loading and elution time, and separation time. On the basis of the fact that loading speed is at $1 \mathrm{~mL} / \mathrm{min}$, total run times could be quite long when large sample volume (i.e. $20-50 \mathrm{~mL}$ ) is used to improve detection limits. To shorten the time between injections, "look ahead" mode can be enabled so that the autosampler sets up for the next injection during the current run. Although the "look ahead" mode can be used to incorporate autosampler preparation and sample withdraw time into chromatography time, the sample is still aspired into the sample loop during the next run cycle. In addition, the dead volume of large sample loops ( $>5 \mathrm{ml}$ ) creates a massive delay of gradient changes and increases the uncertainty of mobile phase mixing.

In the present study, default autosampler program commands were modified from 1-draw sample, 2-move to injection port, 3-aspire sample, 4-inject and 5- 
washing to 1-inject, 2-wait, 3-switch injection valve to load, 4-washing, 5-draw sample, 6-move to injection port, and 7-aspire sample. The amount of time that the modified autosampler program took was compared with those needed for the default injection mode and "look ahead" mode. Efficiencies for the washing steps of injection syringe and sample loop which lead to carryover were also investigated among the default autosampler program, look ahead, and the modified autosampler program for all the compounds in laboratory routine analysis of water samples.

\subsection{Why is pharmacokinetics under the "worst case scenario" important?}

First of all, pharmacokinetic data describing the uptake and depuration of pharmaceuticals by aquatic species are extremely limited compared to those in mammals (Paterson and Metcalfe 2008; Meredith-Williams et al. 2012), thus it is important to study the uptake and depuration rates in systems that may represent "worst case exposure" without compromising the environmental relevance (Fick et al. 2010). Most aquatic monitoring studies designed to simulate exposure under environmentally relevant concentrations were performed in artificially clean matrices (Nakamura et al. 2008; Paterson and Metcalfe 2008; Hoang et al. 2011; Meredith-Williams et al. 2012), often with exposure concentrations still higher than those would be found under natural conditions by one or more orders of magnitude (Paterson and Metcalfe 2008; Hoang et al. 2011). Although this approach maximizes the chances of quantifying any compounds that might accumulate with confidence, results are often not in good agreement with those 
from the field (Nakamura et al. 2008; Paterson and Metcalfe 2008; MeredithWilliams et al. 2012). Reclaimed water produced by conventional primary and secondary treatment offers good surrogacy for a worst case exposure scenario and could be used to predict the extent of bioconcentration and toxicological thresholds in aquatic organisms without resourcing to unrealistic concentrations (Paterson and Metcalfe 2008).

\subsection{Identification of pharmaceutical metabolites in reclaimed water}

Numerous analytical instruments have been applied to investigate the occurrence of parent drugs in a variety of environmental matrices (Vanderford et al. 2003; Davis et al. 2006; Kwon and Armbrust 2006; Ramirez et al. 2007; Schultz et al. 2010; Wang and Gardinali 2012). In contrast, little attention has been paid to the identification, let alone quantification, of pharmaceutical metabolites (Celiz et al. 2009) because there could be numerous metabolites from one parent drug and it is also practically impossible to purchase or synthesize standards for all the metabolites, not to mention the economical unsoundness. As a result, current targeted analysis of metabolites using tandem mass spectrometry is only limited to pharmaceuticals whose metabolites are well studied from clinical data and standards for the metabolites are readily available to purchase (Miao and Metcalfe 2003; Gobel et al. 2004; Miao et al. 2005). However, the increasing number of studies reporting the presence of metabolites in the environment underlined the urge to include metabolites as an important part of routine analysis of pharmaceuticals. For instance, several LC-MS 
methods have been developed to determine carbamazepine, one of the most frequently detected pharmaceuticals in WWTP effluents and surface water, along with its major phase I metabolites in aqueous samples (Miao and Metcalfe 2003; Miao et al. 2005). Interestingly, metabolite 10,11-dihydro-10,11dihydroxycarbamazepine was found to be the dominant analyte which had a concentration 3 to 4 times higher than that of carbamazepine in wastewater (Miao and Metcalfe 2003). A significant increase of the parent drug was also observed in the treated wastewater relative to concentrations in the untreated wastewater, suggesting that some phase II metabolites could have been transformed to free form by cleavage of the conjugates during the treatment. The increase of the parent drug is likely related to the fact that hydroxylated metabolites of carbamazepine occur primarily in conjugated forms such as glucuronidation in body fluids (Miao et al. 2005). Therefore, it is increasingly important to include phase II metabolites when assessing the occurrence, fate and transport of pharmaceuticals in the environment.

\subsection{Driving Hypothesis}

The primary driver is the concern that long-term exposure to low levels of pharmaceutical residues could have adverse effects on aquatic organisms.

\subsection{Objectives}

The main objectives of the present study are to develop sensitive and robust analytical methods using mass spectrometry to investigate the occurrence of 
pharmaceuticals and metabolites in the environment, and to understand the uptake and depuration of pharmaceuticals in biological tissues. The ultimate goal is to assess the ecological risks associated with the exposure.

In chapter 2, an LC-MS/MS method for the analysis of 11 target pharmaceuticals in biological tissues (fish), reclaimed water and surface water is described. BAFs for the detected pharmaceuticals in mosquito fish sampled from a freshwater pond directed affected by reclaimed water were calculated using the developed method.

Chapter 3 describes the modification based on existing on-line SPE LC-MS/MS to improve the throughput and to reduce carryover.

Chapter 4 explains the uptake and depuration as well as bioconcentration factors of pharmaceuticals in reclaimed water by mosquito fish under worst-case exposure scenario.

Chapter 5 describes the exploration of using liquid chromatography ultra-high resolution Orbitrap mass spectrometry to identify and quantify phase II pharmaceutical metabolites in reclaimed water.

In Chapter 6, the potential risks of five bioaccumulative pharmaceuticals including caffeine, carbamazepine, diltiazem, diphenhydramine and ibuprofen detected in reclaimed water were assessed using probabilistic approaches. 


\section{CHAPTER 2}

Analysis of selected pharmaceuticals in fish and the fresh water bodies directly affected by reclaimed water using liquid chromatography-tandem mass spectrometry

(Jian Wang, Piero Gardinali, Analytical and Bioanalytical Chemistry (2012)

404:2711-2720) 


\subsection{Introduction}

Because of increasing reports of their occurrence in water, wastewater, soil, sediment and biosolids (Daughton and Ternes 1999; Heberer 2002; Boyd et al. 2003; Ramirez et al. 2007; Ramirez et al. 2009), pharmaceuticals including drugs and their active metabolites have been recognized as emerging environmental contaminants. Municipal wastewater has been identified as one of the main routes bringing these pharmaceuticals into the environment (Daughton and Ternes 1999). This is likely because wastewater treatment plants (WWTPs) are not specifically designed to remove most of the pharmaceuticals, thus these compounds are continuously released into reclaimed and surface waters in a wide range of concentrations (Heberer 2002; Boyd et al. 2003). Fish are sensitive indicators for substances that enter aquatic ecosystems (EPA 2009). Numerous studies have shown that long-term exposure to pharmaceuticals and personal care products (PPCPs) may result in accumulation of parent compounds, their metabolites, or both in tissues of aquatic organisms, suggesting that further studies on secondary effects of PPCPs on aquatic organisms are necessary (Brooks et al. 2005; Tang et al. 2006; Chu and Metcalfe 2007; Ramirez et al. 2007; Berrada et al. 2008; Nakamura et al. 2008; Zhou et al. 2008; Ramirez et al. 2009; Smith et al. 2009).

On the basis of the complexity of environment matrices and the multiple functionalities of emerging contaminants as target compounds, recently described analytical protocols for the determination of PPCPs in both 
wastewaters (Segura 2007; Lajeunesse et al. 2008; Segura 2009) and biological organisms (Tavazzi et al. 2002; Brooks et al. 2005; Chu and Metcalfe 2007; Berrada et al. 2008) have focused on rather specific classes of compounds. Antidepressants (Brooks et al. 2005; Chu and Metcalfe 2007; Lajeunesse et al. 2008) and antibiotics (Segura 2007; Berrada et al. 2008; Segura 2009) are the two classes that have been studied the most. As the list of compounds found in field collected fish samples expands, the need to develop simultaneous screening methods for multiple classes of drug residues increases as well (Balmer et al. 2004; Duedahl-Olesen et al. 2005; Buser et al. 2006; Rudel et al. 2006; Mottaleb et al. 2009). At present, the general approach employed for the analysis of multi-class pharmaceuticals in fish involves extraction of homogenized tissue with 1:1 mixture of $0.1 \mathrm{M}$ acetic acid and methanol (Ramirez et al. 2007), or acetonitrile combined with limited cleanup to back-extract lipid material followed by liquid chromatography-mass spectrometry (LC-MS) analysis (Smith et al. 2009). Additionally, the use of solid-phase microextraction (SPME) techniques has also been explored as surrogates to estimate the potential occurrence and uptake of pharmaceuticals in living fish by assuming similar partition behavior between the SPME devices and the fish tissues placed in contaminated environments (Zhou et al. 2008).

Accelerated solvent extraction (ASE) involving high pressure and temperature has been shown to efficiently extract a wide range of compounds from fish tissues (Draisci et al. 1998; Datta et al. 2002; Tavazzi et al. 2002; Wahlen 2004; 
Chu and Metcalfe 2007; Haglund et al. 2007; Berrada et al. 2008; Llorca et al. 2009; Losada et al. 2009; Lund et al. 2009). Meanwhile, HLB polymeric cartridges are routinely used as SPE sorbent for cleanup of complex environmental samples prior to LC-MS analysis. However, on the basis of considerable variation in lipophilicity and $\mathrm{pKa}$ among different classes of pharmaceuticals, optimum cleanup efficiency can be challenged by differential retention behavior of target analytes on the sorbent. In contrast, mixed-mode cation exchange (MCX) cartridges can accommodate both neutrals and cations providing better selectivity during elution steps. For instance, Chu et al (Chu and Metcalfe 2007) developed a method to determine paroxetine, fluoxetine and its metabolite in fish tissue using accelerated solvent extraction followed by MCX cleanup offering better recoveries and minimized matrix interferences. Because of the capabilities mentioned above, mixed-mode MCX cartridges will likely provide the needed retention for the multiple classes of pharmaceuticals proposed herein.

The objectives of this study were to develop a comprehensive LC-MS/MS method for the analysis of pharmaceuticals representing multiple therapeutic classes in fish tissue, reclaimed and surface water, and to achieve better sensitivity and recoveries for the determination of pharmaceuticals in biological tissues by using a combination of accelerated solvent extraction followed by mixed-mode SPE cleanup and LC-MS/MS detection. 


\subsection{Experimental}

\subsubsection{Reagents, standards and solutions}

The LC-MS grade methanol, water and formic acid used for mass spectrometry analysis and the Optima grade methylene chloride and acetonitrile used for ASE extraction were purchased from Fisher Scientific (Atlanta, GA). Distilled water for SPE cleanup was purified and deionized with a Barnstead Nanopure water purification system. Reference standards lincomycin, trimethoprim, caffeine, sulfamethoxazole, diphenhydramine, diltiazem, carbamazepine, fluoxetine, erythromycin, norfluoxetine and sertaline were purchased in the highest available purity (Sigma-Aldrich, Milwaukee, WI). Five surrogates, covering the range of functionalities were used for the method. Caffeine $-{ }^{13} \mathrm{C}_{3}$ was purchased from Cambridge Isotopes Lab. Inc. (Andover, MA). Sulfamethoxazole- $\mathrm{d}_{4}$, erythromycin $-{ }^{13} \mathrm{C}, \mathrm{d}_{3}$ and paroxetine- $\mathrm{d}_{4}$ were purchased from Toronto Research Chemicals Inc. (North York, Ontario). Carbamazepine- $d_{10}$ was purchased from C/D/N Isotopes Inc. (Pointe-Claire, Quebec). Fluoxetine- $d_{6}$ was purchased from Cerilliant Corp. (Round Rock, TX). All stock solutions and working solutions were made in methanol and stored at $-20^{\circ} \mathrm{C}$ in the dark to prevent degradation. The $0.1 \%$ formic acid solution used for mobile phase was prepared daily before analysis. Ancillary solutions used as modifiers, i.e. acetic acid ( $\mathrm{pH} 3.2), 5 \%$ ammonium hydroxide in methanol and methanol:0.1\% formic acid 50:50 (v/v) were prepared daily. 


\subsubsection{Sample collection and storage}

Water and mosquito fish (Gambusia holbrooki) were collected from a freshwater pond and a saltwater marsh located at Florida International University Biscayne Bay Campus (North Miami Beach, FL). The saltwater tidal marsh is not impacted by reclaimed water and is hydrologically connected with Biscayne Bay while the freshwater pond is isolated from the tidal influence of the bay and continuously receives storm water runoff and reclaimed water from a local WWTP through daily sprinkler irrigation. Therefore, the organisms living in this pond are likely to be chronically exposed to the contaminants from reclaimed water. Seven composite mosquito fish samples were collected from each sampling site using unbaited minnow traps deployed at depth between 20 and $30 \mathrm{~cm}$. The sizes of the fish collected in the traps ranged from $2.0-4.2 \mathrm{~cm}$ (total length) and individuals weighed from 0.3-2.1 g. All tissue samples were brought to the laboratory, sorted, classified and stored at or below $-20{ }^{\circ} \mathrm{C}$ until time of analysis.

Fish were pooled to obtain at least $30 \mathrm{~g}$ of material and homogenized with a ULTRA TURRAX IKA T18 stainless steel tissuemiser (Wilmington, NC) set to rotate at $10,000 \mathrm{rpm}$. The homogenates were stored at $-20^{\circ} \mathrm{C}$ and thawed at $4{ }^{\circ} \mathrm{C}$ for approximately $10 \mathrm{~h}$ before extraction. Reclaimed water (1-Liter) was directly drawn from irrigation sprinklers during a period of two months, while pond water samples were collected in 1-Liter pre-cleaned amber glass bottles at the site where fish were collected. All water samples were transported to the laboratory and stored in dark at or below $4{ }^{\circ} \mathrm{C}$ until they were filtered and processed. Water 
extraction was conducted within 7 days of collection to prevent losses on the basis of biodegradation.

\subsubsection{Animal care and use}

All animal experiments were conducted in accordance with the Institutional Animal Care and Use Committee of Florida International University (\#A3096-01).

\subsubsection{Solid phase extraction for water samples}

To remove suspended materials, water samples were filtered through $0.5 \mu \mathrm{m}$ pre-combusted glass fiber filters (GE Water \& Process Technologies, Trevose, PA) within $24 \mathrm{~h}$ after collection. $50 \mu \mathrm{L}$ of surrogate standards solution were added to each $250 \mathrm{~mL}$ of filtered reclaimed water or pond water sample. Oasis HLB (3cc/60mg, Waters Corp., Franklin, MA) cartridges were conditioned with 3 $\mathrm{mL}$ of methanol followed by $3 \mathrm{~mL}$ of distilled water. Solid phase extraction was performed on an ALLTECH 12-port vacuum manifold (Deerfield, IL). After the samples had passed through the HLB cartridges, they were washed with $4 \mathrm{~mL}$ of $5 \%$ methanol in water prepared daily. The analytes were eluted with $3 \mathrm{~mL}$ of methanol. Each methanol eluent was mixed with $50 \mu \mathrm{L}$ of paroxetine- $\mathrm{d}_{4}$ internal standard solution and evaporated to dryness under a gentle stream of purified nitrogen gas. Dried residues were reconstituted with $200 \mu \mathrm{L}$ of methanol:0.1\% formic acid 50:50 (v/v). After brief sonication and vortex, the samples were ready for LC-MS/MS analysis. 


\subsubsection{ASE extraction for fish tissues}

Fish samples were extracted using a Dionex ASE 200 accelerated solvent extraction system (Dionex Corp., Sunnyvale, CA). $25 \mathrm{~g}$ of $\mathrm{Na}_{2} \mathrm{SO}_{4}$ were placed in the 33-mL stainless steel extraction cell containing a glass fiber filter in the outlet side. Approximately $1 \mathrm{~g}$ of fish homogenate was placed on top of the $\mathrm{Na}_{2} \mathrm{SO}_{4}$. Five surrogates including caffeine $-{ }^{13} \mathrm{C}_{3}(412.0 \mathrm{ng})$, Sulfamethoxazole- $\mathrm{d}_{4}(100.0$ $n g)$, carbamazepine- $d_{10}(29.5 \mathrm{ng})$, fluoxetine- $\mathrm{d}_{6}(31.0 \mathrm{ng})$, and erythromycin $-{ }^{13} \mathrm{C}$, $d_{3}$ (100.0 ng) were added to each sample. The cell was topped with another glass fiber filter, firmly capped and extracted. Methylene chloride was employed as the extraction solvent. The ASE conditions are as follows: oven temperature $80^{\circ} \mathrm{C}$; pressure $1500 \mathrm{psi}$; heat for $5 \mathrm{~min}$; 1 static cycle; static time $10 \mathrm{~min}$; flush volume $60 \%$ and purge for $120 \mathrm{sec}$. After each extraction cycle, $30 \mathrm{~mL}$ methylene chloride extract was flushed into a $60-\mathrm{mL}$ glass vial and dried under gentle nitrogen stream. The dried extract was then reconstituted with $30 \mathrm{~mL}$ of acetic acid $(\mathrm{pH} \mathrm{3.2)}$ in water. The glass vial was sonicated for 10 min and the sample was ready for MCX-SPE cleanup.

\subsubsection{Cleanup for fish tissues}

3cc/60mg Oasis MCX cartridges (Waters Corp., Franklin, MA) were employed for the fish extract cleanup. The cartridges were conditioned with $3 \mathrm{~mL}$ of methanol followed by $3 \mathrm{ml}$ of acetic acid in water ( $\mathrm{pH} \mathrm{3.2).} \mathrm{Care} \mathrm{was} \mathrm{taken} \mathrm{not} \mathrm{to} \mathrm{dry} \mathrm{the}$ cartridges during loading process. A cartridge flow rate of less than $1 \mathrm{~mL} / \mathrm{min}$ 
was operated under the vacuum of 20 psi. Once the samples had passed through the cartridges, they were washed with $2 \mathrm{~mL}$ acetonitrile and dried for 5 min. The cartridges were then eluted with $3 \mathrm{~mL}$ of $5 \%$ ammonium hydroxide in methanol. $50 \mu \mathrm{L}$ of paroxetine- $\mathrm{d}_{4}$ internal standard solution were added, and samples were dried using a centrifuge concentrator (Labconco Corp., Kansas City, MO). $200 \mu \mathrm{L}$ of methanol:0.1\% formic acid 50:50 (v/v) were added to each sample. After 10 min sonication and 1 min of vortex, samples were transferred and analyzed by LC-MS/MS.

\subsubsection{LC-MS/MS analysis}

The liquid chromatography system consists of a Thermo PAL CTC autosampler and a quaternary Thermo Scientific Accela pump. Analytes were separated on a Hypersil GOLD $50 \times 2.1 \mathrm{~mm}, 3 \mu \mathrm{m}$ particle size and 115 pore size column, from Thermo Scientific (Bellefonte, PA). A simple binary gradient consisting of A: $0.1 \%$ formic acid in water $(\mathrm{v} / \mathrm{v})$ and B: $100 \%$ methanol was employed for chromatographic separation. The gradient was (methanol \%): 0 min 3\%, 4 min $3 \%, 10 \min 97 \%, 13 \min 3 \%$ and $16 \min 3 \%$. The flow rate was maintained at $300 \mu \mathrm{L} / \mathrm{min}$. The injection volume was $20 \mu \mathrm{L}$.

All the samples were analyzed with a Thermo TSQ Quantum Access triple quadrupole $(\mathrm{QqQ})$ mass spectrometry equipped with a heated electrospray ion source (HESI). Tandem mass spectrometry detection was performed in selected reaction monitoring (SRM) mode. Collision energy (CE) and tube lens voltage for 
each compound were optimized through direct infusion into mass spectrometer at concentration of $1 \mu \mathrm{g} / \mathrm{mL}$ and at the flow rate of $15 \mu \mathrm{L} / \mathrm{min}$. Additional instrumental parameters for all analytes were as follows: Spray voltage at 3500 $\mathrm{V}$, Capillary temperature and Vaporizer temperature at $300{ }^{\circ} \mathrm{C}$; Sheath gas $\left(\mathrm{N}_{2}\right)$ and Aux gas $\left(\mathrm{N}_{2}\right)$ at 30 arbitrary units; Ion sweep gas $\left(\mathrm{N}_{2}\right)$ at 10 arbitrary units. Scan time was set to $0.5 \mathrm{~s}$. Precursor ions, product ions and SRM transition parameters are listed in Table 2.1.

Table 2.1 SRM transition parameters

\begin{tabular}{ccccc}
\hline Compounds & SRM 1 & $\begin{array}{c}\text { Collision } \\
\text { energy } \\
(\mathrm{V})\end{array}$ & SRM 2 & $\begin{array}{c}\text { Collision } \\
\text { energy } \\
(\mathrm{V})\end{array}$ \\
\hline lincomycin & $407 \rightarrow 126$ & 31 & $407 \rightarrow 359$ & 18 \\
trimethoprim & $291 \rightarrow 230$ & 24 & $291 \rightarrow 260$ & 25 \\
caffeine & $195 \rightarrow 138$ & 18 & $195 \rightarrow 110$ & 21 \\
sulfamethoxazole & $254 \rightarrow 156$ & 17 & $254 \rightarrow 108$ & 29 \\
diphenhydramine & $256 \rightarrow 167$ & 15 & $256 \rightarrow 152$ & 40 \\
diltiazem & $415 \rightarrow 178$ & 25 & $415 \rightarrow 150$ & 39 \\
carbamazepine & $237 \rightarrow 194$ & 19 & $237 \rightarrow 192$ & 21 \\
erythromycin & $717 \rightarrow 540$ & 18 & $717 \rightarrow 558$ & 15 \\
fluoxetine & $310 \rightarrow 148$ & 8 & $310 \rightarrow 44$ & 12 \\
norfluoxetine & $296 \rightarrow 134$ & 6 & $296 \rightarrow 31$ & 46 \\
sertraline & $306 \rightarrow 275$ & 12 & $306 \rightarrow 159$ & 28 \\
\hline
\end{tabular}




\subsubsection{Matrix effects}

Matrix effects could have a severe detrimental effect in LC-MS/MS analysis especially when coupled with ESI sources (Vanderford et al. 2003; Segura 2007). Both signal suppression and enhancement are commonly observed on the basis of co-eluting matrix interferences. The exact mechanism still remains unknown but it has been widely discussed in previous work with respect to PPCPs (Vanderford et al. 2003; Renew and Huang 2004; Segura 2007).

In order to evaluate matrix effects, replicates of clean fish tissues $(1 \mathrm{~g})$ were extracted and cleaned up as described above. One of the extracts was spiked with a known amount of analytes and surrogates and brought to a final volume of $200 \mu \mathrm{L}$ with methanol:0.1\% formic acid (50/50, v/v). Simultaneously, a methanol:0.1\% formic acid $(50 / 50, \mathrm{v} / \mathrm{v})$ solution spiked with the same concentration of analytes and surrogates was analyzed as a matrix-free reference sample. Matrix effects were evaluated using the following equation (Segura 2007):

$$
\text { Matrix effects (\%) }=\left(\frac{R_{m-s}-R_{m}}{R_{0}}-1\right) \times 100
$$

where $R_{m+s}$ is the response ratio of the analyte in spiked matrix, $R_{m}$ is the response ratio of the analyte in unspiked matrix and $R_{0}$ is the response ratio of the analyte in matrix-free reference sample. 


\subsection{Results and Discussion}

The target compounds were selected based on the results of an EPA pilot study for PPCPs in fish tissue (EPA 2009) and a previously reported PPCP screening method (Ramirez et al. 2007). According to the EPA pilot study, carbamazepine, diltiazem, diphenhydramine, fluoxetine, norfluoxetine and sertraline have been detected in fish collected from 5 effluent-dominated streams in various regions of the United States. Ramirez et al. (Ramirez et al. 2007) have also reported the presence of diphenhydramine, diltiazem, carbamazepine and norfluoxetine in fish collected from an effluent-dominated stream in Texas. In addition to the target compounds mentioned above, other pharmaceuticals such as trimethorprim, caffeine, sulfamethoxazole and erythromycin that have been routinely detected in the reclaimed water used for ground irrigation at Florida International University Biscayne Bay Campus (North Miami Beach, FL) were also included in this study to assess potential accumulation from the reclaimed water (Wang and Gardinali 2012).

\subsubsection{Mass spectrometry}

In order to achieve similar ionization behavior as those expected during real sample analysis, optimization of analyte responses was performed while the mobile phase was infused along with the standard solution through a tee connector at a speed of $50 \mu \mathrm{L} / \mathrm{min}$. The most abundant molecular ion was selected as the precursor ion for that particular analyte. With the exception of 
erythromycin, protonated $[\mathrm{M}+\mathrm{H}]^{+}$was found to be the most abundant precursor ion for all analytes. $\left[\mathrm{M}+\mathrm{H}-\mathrm{H}_{2} \mathrm{O}\right]^{+}$was found to be the most abundant precursor ion for erythromycin which was in agreement with Hirsh et al.,(Hirsch et al. 1999) who showed that erythromycin has already lost a water molecule when present in the aquatic environment. Once the precursor ion has been identified, Q3 was scanned to define product ions and to optimize the collision energy for each compound. Two SRM transitions were selected to avoid false positives and to give 4 identification points in accordance with EU Commission Decision 2002/657/EC (2009). All optimized parameters can be found in Table 1.

Both electrospray ionization (ESI) and heated electrospray ionization (HESI) probes were tested to obtain optimal ionization efficiency. HESI is designed to use ESI in combination with heated auxiliary gas that transforms ions in solution into ions in the gas phase more efficiently. As shown in Figure 2.1, HESI probe showed at least 2-4 times improvement over ESI for all the compounds, in particular for early eluting compounds such as lincomycin, caffeine, and trimethoprim mainly because of better peak shapes and narrower peak width. Therefore, HESI was selected as the ion source in this study. 


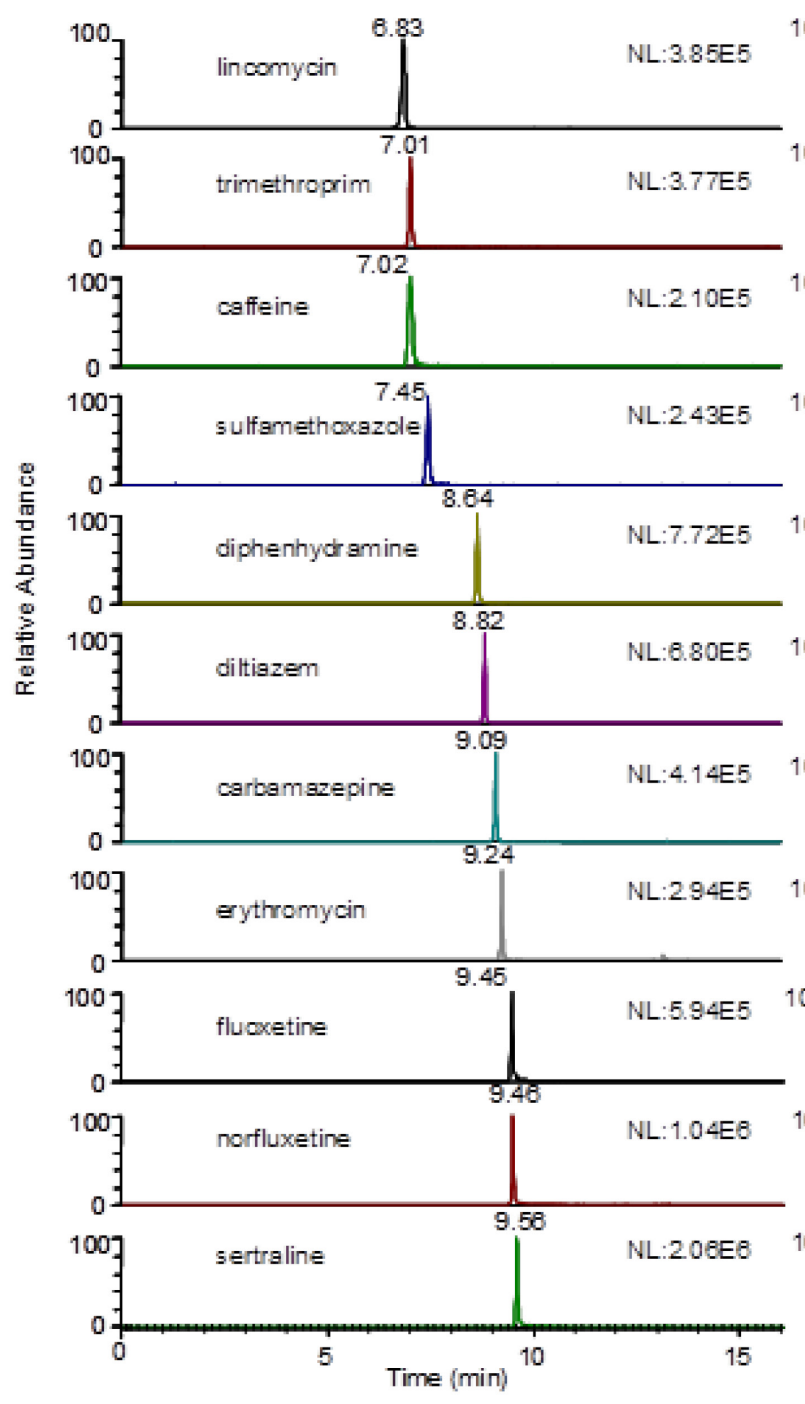

(A)

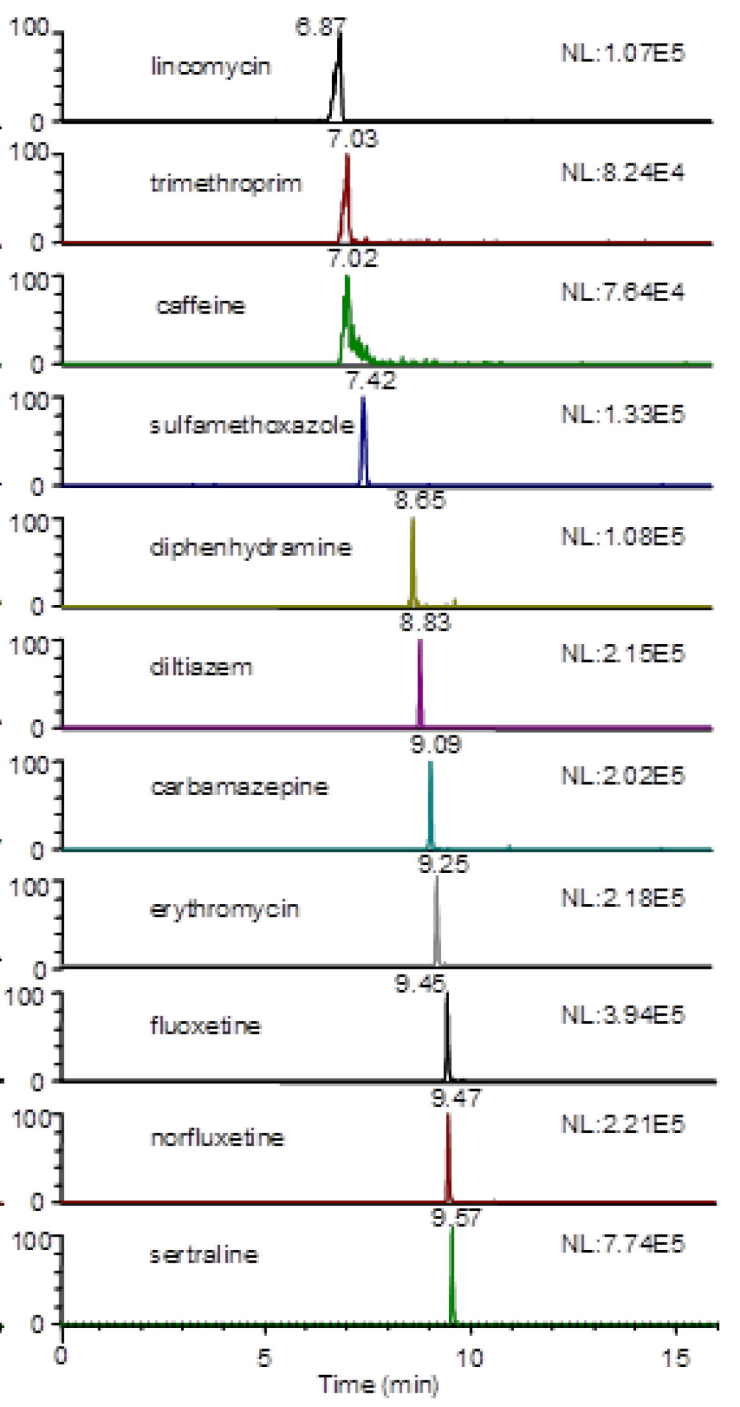

(B)

Figure 2.1 Representative chromatograms of all selected pharmaceuticals

standards on HESI (A) and ESI (B) sources at the same spiking level

\subsubsection{Accelerated solvent extraction for fish tissues}

The most commonly used methods for extracting multiclass pharmaceuticals from fish tissues involve sonicating or vigorously shaking tissue homogenates with organic solvents such as acetonitrile and methanol followed by direct LC-MS analysis (Tang et al. 2006; Ramirez et al. 2007; EPA 2009; Smith et al. 2009). 
However, in order to improve the extraction efficiency and method throughput, ASE was used because of its advantages over traditional methods, including automated extraction, higher recoveries, and smaller volume of extraction solvents (Draisci et al. 1998; Datta et al. 2002; Tavazzi et al. 2002; Wahlen 2004; Chu and Metcalfe 2007; Haglund et al. 2007; Berrada et al. 2008; Llorca et al. 2009; Losada et al. 2009; Lund et al. 2009). The first challenge when developing ASE method is to choose the appropriate extraction solvent. Various solvents including methanol, acetone, acetonitrile, and methylene chloride were tested for fish homogenate samples $(1 \mathrm{~g})$ fortified with all the analytes. The final selection of the extraction solvent was based on the "absolute recovery" calculated by subtracting the peak area of unspiked sample from that of the spiked sample. Absolute recoveries in different solvent systems are shown in Figure 2.2, where methylene chloride showed the highest absolute recoveries for all the analytes except for lincomycin and erythromycin. Other ASE conditions such as oven temperature, pressure, static time and cycles, heat-up time and flush volume were selected with slight modifications according to previously reported methods (Chu and Metcalfe 2007). 


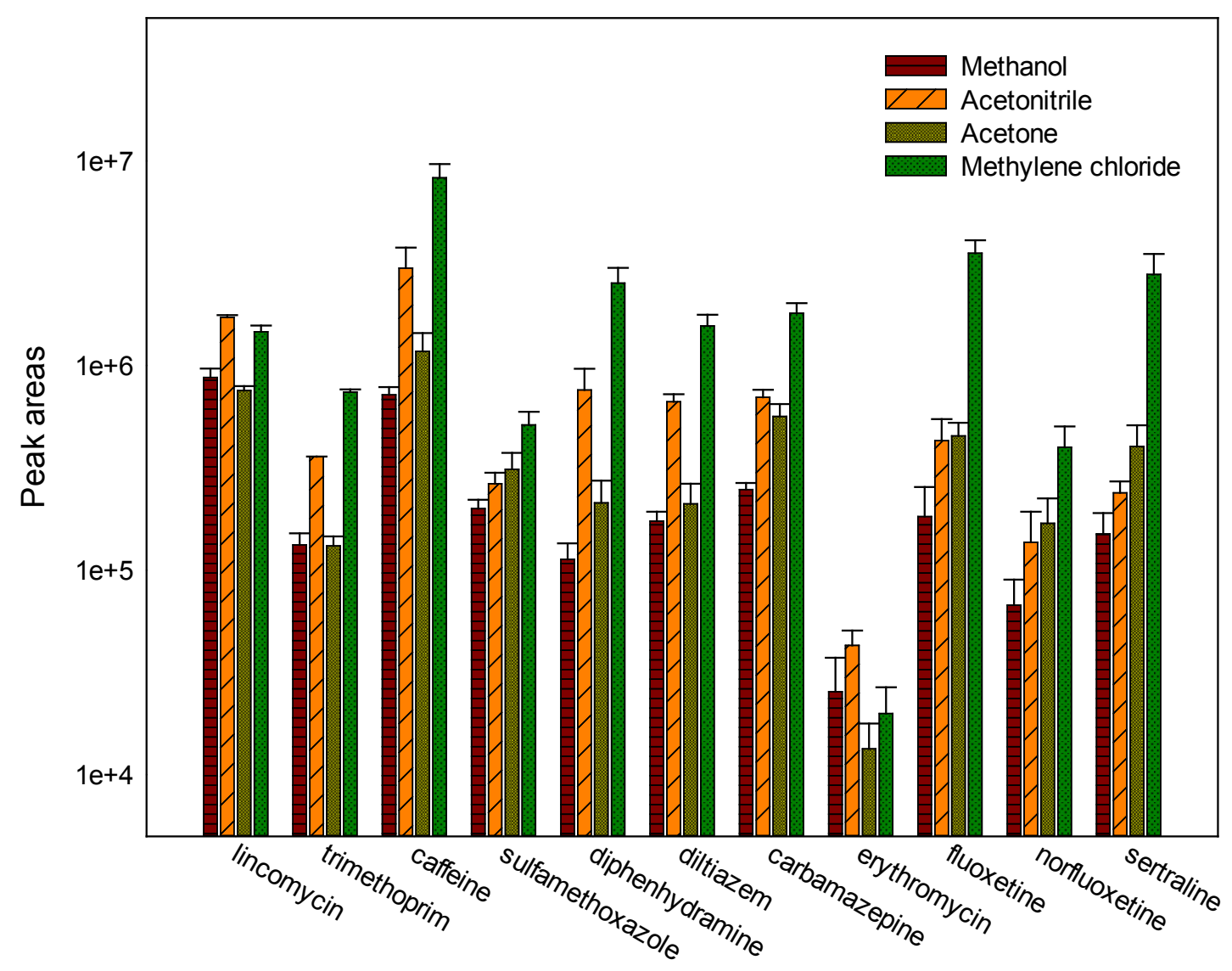

Figure 2.2 ASE solvent selection based on absolute recoveries

\subsubsection{SPE cleanup}

Although trace analysis at $\mathrm{ng} / \mathrm{g}$ level can be achieved when using mass spectrometry as the detection method, matrix effects are still problematic on the basis of co-eluting matrix components during the extraction procedures without additional cleanup steps (Ramirez et al. 2007; EPA 2009). Gel permeation chromatography (GPC) is generally applied to remove lipid contents from biological tissues. However, this method requires large volume of organic solvent 
and an extra cleanup step is still needed to make the sample suitable for LC-MS analysis (Chu and Metcalfe 2007). In contrast, mixed mode cation-exchange (MCX) SPE has been shown to be a successful alternative to cleanup complicated matrices such as fish tissues while still maintaining optimum recoveries of analytes with considerable ranges of $\mathrm{pKa}$ and lipophilicity (Chu and Metcalfe 2007). MCX cartridges can provide much cleaner extract than regular HLB cartridges because organic solvents such as methanol or acetonitrile can be used to wash cartridges and eliminate interferences without losing the selectively retained analytes for further elution. The only requirement is that the samples need to be acidified in order for analytes to be retained on the sorbent by cation exchange reactions. In addition, care must be taken during the $\mathrm{pH}$ adjustment because analytes could be lost during the loading and washing steps if the $\mathrm{pH}$ is too low (Chu and Metcalfe 2007). On the basis of the fact that sulfamethoxazole has the lowest pKa of 5.8 among all the analytes (Qiang and Adams 2004), ASE extract was adjusted with acetic acid to $\mathrm{pH} 3.3$ which is approximately 2 units below the $\mathrm{pKa}$ of sulfamethoxazole. Five solvents including methanol, acetonitrile, acetone, methylene chloride and hexane:methylene chloride (50/50, $\mathrm{v} / \mathrm{v}$ ) mixture were tested as the wash solvents. Individual analyte recoveries in each solvent system are plotted in Figure 2.3, where error bars represent standard deviations from average recoveries $(n=3)$. Because acetonitrile is a stronger eluent than methanol (Nelis 1983), higher recoveries were expected from the methanol wash. However, acetontitrile was found to be the most 
effective solvent at removing interferences from the cartridge while offering maximum recovery and good overall precision.

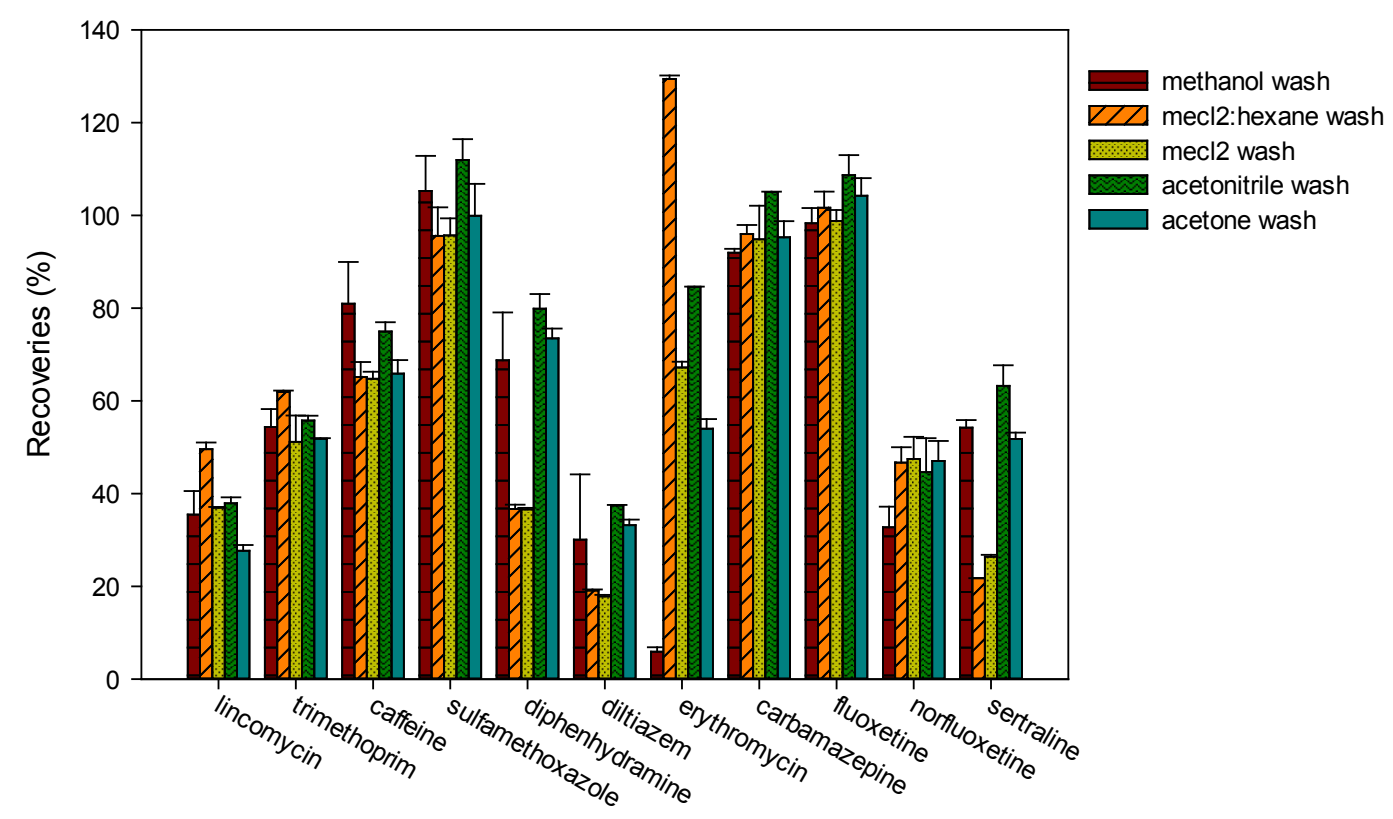

Figure 2.3 Averaged $(n=3)$ individual analyte recoveries in each wash solvent system

\subsubsection{Matrix effects}

All compounds were analyzed for potential matrix effects in HESI positive mode and results are shown in Table 2.2 along with the statistically derived MDLs $(n=7)$. Positive values indicate signal enhancement and negative values indicate signal suppression on the basis of the matrix effects. As shown in Table 2.2, caffeine and erythromycin showed moderate signal enhancements. Similar results were also observed by Ramirez et al. (Ramirez et al. 2007). Other compounds showed various degrees of signal suppression. 
Table 2.2 Observed MDLs Matrix Effects and recoveries in fish tissue

\begin{tabular}{ccccc}
\hline & MDL (ng/g) & $\begin{array}{c}\text { Ramirez's MDL(ng/g) } \\
\text { (Ramirez et al. 2007) }\end{array}$ & $\begin{array}{c}\text { Matrix Effects } \\
(\%)\end{array}$ & Recovery (\%) \\
\hline lincomycin & 1.05 & 5.53 & $-38 \pm 0.2$ & $63 \pm 8$ \\
trimethoprim & 1.03 & 2.15 & $-65 \pm 1$ & $83 \pm 4$ \\
caffeine & 0.81 & 3.93 & $33 \pm 3$ & $64 \pm 13$ \\
sulfamethoxazole & 0.84 & 2.29 & $-54 \pm 1$ & $44 \pm 4$ \\
diphenhydramine & 0.08 & 0.05 & $-40 \pm 1$ & $85 \pm 4$ \\
diltiazem & 0.11 & 0.12 & $-95 \pm 1$ & $60 \pm 16$ \\
carbamazepine & 0.10 & 0.54 & $-26 \pm 0.4$ & $61 \pm 0.5$ \\
erythromycin & 0.51 & 6.42 & $20 \pm 0.5$ & $28 \pm 0.4$ \\
fluoxetine & 1.19 & 6.73 & $-5 \pm 5$ & $66 \pm 12$ \\
norfluoxetine & 0.41 & 2.90 & $-42 \pm 5$ & $19 \pm 10$ \\
sertraline & 0.26 & 3.57 & $-26 \pm 2$ & $80 \pm 1$ \\
\hline
\end{tabular}


For water samples, matrix effects were evaluated using the same procedure as for fish tissues (Table 2.3). Organic matter in surface waters has shown to play an important role on matrix effects (Renew and Huang 2004). The overall results indicate that reclaimed water showed more pronounced effects than pond water for most of the compounds. Signal enhancement was observed on 7 compounds in reclaimed water samples while only 2 compounds showed signal enhancement in the pond water samples.

\subsubsection{Analytical performance}

Analyte concentrations in fish tissues were determined based on response factors (RFs) of the target compounds relative to the surrogate internal standards. This approach can be used for most trace analysis, as it doesn't require blank matrix and greatly alleviates the signal suppression or enhancement arising from matrix effects that can affect the sensitivity and response of the mass spectrometer in unpredictable ways.

Method detection limits (MDLs) were used to evaluate the analytical performance in different matrices and reported in Table 2.2 and Table 2.3. MDL represents the lowest concentration of the analyte that can be measured and reported with $99 \%$ confidence in a given matrix is greater than zero (1986). It has been discussed and generally assumed that experimentally derived MDLs in a given matrix are appropriate for establishing detection threshold in environmental analysis (Ramirez et al. 2007; Mottaleb et al. 2009; Ramirez et al. 2009). 
Table 2.3 Observed MDLs matrix effects and recoveries in pond water and reclaimed water

\begin{tabular}{|c|c|c|c|c|c|c|}
\hline & \multicolumn{3}{|c|}{ Pond Water } & \multicolumn{3}{|c|}{ Reclaimed Water } \\
\hline & $\begin{array}{c}\text { MDL } \\
(\mathrm{ng} / \mathrm{L})\end{array}$ & $\begin{array}{c}\text { Matrix } \\
\text { Effects } \\
(\%)\end{array}$ & $\begin{array}{c}\text { Recovery } \\
(\%)\end{array}$ & $\begin{array}{c}\text { MDL } \\
(\mathrm{ng} / \mathrm{L})\end{array}$ & $\begin{array}{c}\text { Matrix } \\
\text { Effects } \\
(\%)\end{array}$ & Recovery (\%) \\
\hline lincomycin & 3.3 & $263 \pm 14$ & $136 \pm 14$ & 10.9 & $268 \pm 59$ & $152 \pm 12$ \\
\hline trimethoprim & 2.6 & $64 \pm 2$ & $101 \pm 6$ & 266 & $74 \pm 6$ & $69 \pm 4$ \\
\hline caffeine & 7.6 & $-7 \pm 6$ & $101 \pm 8$ & 348 & $33 \pm 6$ & $75 \pm 13$ \\
\hline sulfamethoxazole & 4.6 & $-14 \pm 3$ & $104 \pm 5$ & 67.5 & $9 \pm 4$ & $94 \pm 14$ \\
\hline diphenhydramine & 0.4 & $-41 \pm 0.8$ & $82 \pm 1$ & 53.1 & $-18 \pm 0.3$ & $79 \pm 1$ \\
\hline diltiazem & 0.2 & $-36 \pm 3$ & $68 \pm 0.7$ & 26.9 & $-49 \pm 4$ & $83 \pm 0.2$ \\
\hline carbamazepine & 1.5 & $-18 \pm 1$ & $90 \pm 5$ & 17.8 & $26 \pm 7$ & $117 \pm 23$ \\
\hline erythromycin & 15 & $-2 \pm 4$ & $112 \pm 2$ & 88.0 & $35 \pm 3$ & $115 \pm 8$ \\
\hline fluoxetine & 3.4 & $-3 \pm 4$ & $95 \pm 6$ & 5.0 & $7 \pm 0.9$ & $86 \pm 0.1$ \\
\hline norfluoxetine & 1.0 & $-44 \pm 2$ & $32 \pm 2$ & 1.8 & $-25 \pm 2$ & $21 \pm 0.1$ \\
\hline sertraline & 1.2 & $-9 \pm 3$ & $56 \pm 0.6$ & 4.9 & $-2 \pm 2$ & $40 \pm 0.6$ \\
\hline
\end{tabular}


Seven replicates of $1 \mathrm{~g}$ of clean fish tissues $(n=7)$ were spiked with an appropriate amounts of analytes (for spike levels see Table 2.4) and extracted as described above. Similarly, seven replicates of $250 \mathrm{~mL}$ pond water and $250 \mathrm{~mL}$ reclaimed water were spiked at the same level and subjected to the SPE-LCMS/MS analysis. MDLs were then calculated by multiplying the one-side student's t-value at $99 \%$ confidence by the sample standard deviation derived from the concentrations of 7 replicate spiked samples (1986). Concentrations below MDLs were reported as "not detected". Compared to the previous studies (Ramirez et al. 2007), the protocol demonstrated here offers cleaner fish extracts giving lower MDLs for 10 out of 11 selected compounds. The MDL for diphenhydramine was slightly higher but similar to the value reported by Ramirez et al. (Ramirez et al. 2007). Concentrations of diphenhydramine detected in fish tissues, however, were considerably higher than the calculated MDL. For water samples, MDLs in reclaimed water were significantly higher than those in pond water because of both a more complicated matrix and higher overall concentrations that produced larger standard deviations. The recoveries of norfluoxetine were found at $19 \%$ in fish, $32 \%$ in pond water and $21 \%$ in reclaimed water, respectively. The low recovery is likely on the basis of the lack of methyl group in norfluoxetine increasing the water solubility compared to fluoxetine. 
Table 2.4 Fortification levels for matrix effect assessment, recovery calculation and MDL determination in fish tissues and water samples

\begin{tabular}{|c|c|c|c|c|c|c|}
\hline & \multicolumn{3}{|c|}{ fortification levels in fish tissues (ng/g) } & \multicolumn{3}{|c|}{ fortification levels in water (ng/L) } \\
\hline & $\begin{array}{c}\text { Matrix } \\
\text { effect } \\
\text { assessment }\end{array}$ & $\begin{array}{l}\text { Recovery } \\
\text { calculation }\end{array}$ & $\begin{array}{c}\text { MDL } \\
\text { determination }\end{array}$ & $\begin{array}{c}\text { Matrix } \\
\text { effect } \\
\text { assessment }\end{array}$ & $\begin{array}{c}\text { Recovery } \\
\text { assessment }\end{array}$ & $\begin{array}{c}\text { MDL } \\
\text { determination }\end{array}$ \\
\hline lincomycin & 9.9 & 9.9 & 1.0 & 40.0 & 40.0 & 4.0 \\
\hline trimethoprim & 9.9 & 9.9 & 1.0 & 40.0 & 40.0 & 4.0 \\
\hline caffeine & 39.9 & 39.9 & 4.0 & 160 & 160 & 16.0 \\
\hline sulfamethoxazole & 39.9 & 39.9 & 4.0 & 160 & 160 & 16.0 \\
\hline diphenhydramine & 2.0 & 2.0 & 0.2 & 8.0 & 8.0 & 0.8 \\
\hline diltiazem & 2.0 & 2.0 & 0.2 & 8.0 & 8.0 & 0.8 \\
\hline carbamazepine & 4.0 & 4.0 & 0.4 & 16.0 & 16.0 & 1.6 \\
\hline erythromycin & 20.1 & 20.1 & 2.0 & 80.4 & 80.4 & 8.0 \\
\hline fluoxetine & 60.0 & 60.0 & 6.0 & 240 & 240 & 24.0 \\
\hline norfluoxetine & 30.0 & 30.0 & 3.0 & 120 & 120 & 12.0 \\
\hline sertraline & 19.9 & 19.9 & 2.0 & 80.0 & 80.0 & 8.0 \\
\hline
\end{tabular}




\subsubsection{Analysis of environmental samples}

Mosquito fish (Gambusia holbrooki) sampled from a saltwater marsh not influenced by reclaimed water and a freshwater pond affected by reclaimed water influence were analyzed for target analytes. Reclaimed water and pond water samples collected over a period of 2 months were also concurrently analyzed. Concentrations of target analytes in water samples are summarized in Table 2.5. $73 \%$ of target compounds were consistently detected in reclaimed water samples. However, fluoxetine, diltiazem and erythromycin were not detected in the pond water influenced by reclaimed water. The possible explanation is that these compounds can be rapidly dissipated from the water phase as a result of adsorption to sediment where they seem to be persistent (Andrisano et al. 2001; Kwon and Armbrust 2005; Davis et al. 2006; Kwon and Armbrust 2006). Therefore, it is not surprising that neither fluoxetine nor diltiazem was detected in fish tissues in this study even though they have been reported to accumulate in fish (Brooks et al. 2005; Chu and Metcalfe 2007; Ramirez et al. 2007). As shown in Table 2.6, caffeine, diphenhydramine and carbamazepine were detected in fish tissues from the freshwater pond but no target compounds were detected in those from the saltwater marsh. Bioaccumulation factors for caffeine, diphenhydramine and carbamazepine in mosquito fish (Gambusia holbrooki) were calculated accordingly and found at $29 \pm 26,821 \pm 422$ and $108 \pm 144$, respectively. The calculated BAF for carbamazepine was consistent with literature value (Vernouillet et al. 2010). All the resulting BAFs were higher than 
those calculated based on log $K_{\text {ow }}$ (octanol/water partition coefficient) (Duffy and Jorgensen 2000) and EPA $K_{\text {ow }}$ based Aquatic BioAccumulation Model (KABAM) (1 for caffeine, 93 for diphenhydramine and 14 for carbamazepine) (2009; EPA 2009). Upon plotting a graph of BAF vs log $K_{\mathrm{ow}}$, a positive relationship was observed $\left(R^{2}=0.5665\right)$ which was consistent with previously proposed theory (Gossett et al. 1983). While diphenhydramine and carbamazepine have been previously observed in fish tissues (Ramirez et al. 2007), potential accumulation of caffeine in fish is reported here for the first time.

\subsection{Conclusions}

This study presents the development of a new method for the analysis of selected pharmaceuticals in fish tissue, reclaimed water and surface water directly affected by reclaimed water. Compared to previous methods, accelerated solvent extraction followed by MCX mixed-mode SPE cleanup provided a better alternative on the basis of cleaner extracts giving lower method detection limits. $73 \%$ and $45 \%$ of all target compounds were consistently detected in reclaimed water and surface water, respectively. Caffeine, diphenhydramine and carbamazepine were detected in mosquito fish from the freshwater pond directly affected by reclaimed water influence but they were not detected in those from the saltwater mash. Bioaccumulation factors for caffeine, diphenhydramine and carbamazepine in mosquito fish were also calculated and found at $29 \pm 26,821 \pm$ 422 and $108 \pm 144$, respectively. 
Table 2.5 Summary of concentrations of target compounds in reclaimed water and pond water

\begin{tabular}{|c|c|c|c|c|c|c|c|c|c|c|}
\hline \multirow{2}{*}{ Compounds } & \multicolumn{5}{|c|}{ Reclaimed water (ng/L) $n=17$} & \multicolumn{5}{|c|}{ Pond water (ng/L) $n=9$} \\
\hline & Mean & sd & Max & Min & Median & Mean & sd & $\operatorname{Max}$ & Min & Median \\
\hline lincomycin & ND & & $N D$ & $N D$ & $N D$ & ND & & $N D$ & $N D$ & ND \\
\hline trimethoprim & 338 & 273 & 920 & 16 & 254 & 1.3 & 3.7 & 11 & ND & ND \\
\hline caffeine & 1476 & 1177 & 4315 & 53 & 1217 & 81 & 48 & 172 & 23 & 63 \\
\hline sulfamethoxazole & 241 & 128 & 409 & 3.0 & 263 & 8.0 & 5.2 & 14 & $N D$ & 10 \\
\hline diphenhydramine & 89 & 48 & 179 & 6.3 & 77 & 0.67 & 0.38 & 1.3 & $N D$ & 0.62 \\
\hline diltiazem & 29 & 30 & 111 & $N D$ & 22 & $N D$ & & $N D$ & $N D$ & $N D$ \\
\hline carbamazepine & 97 & 55 & 229 & 20 & 83 & 4.5 & 1.9 & 6.6 & 1.9 & 5.4 \\
\hline erythromycin & 79 & 37 & 141 & $N D$ & 88 & $N D$ & & $N D$ & $N D$ & $N D$ \\
\hline fluoxetine & 9.7 & 5.4 & 24 & $N D$ & 9.7 & $N D$ & & $N D$ & ND & ND \\
\hline norfluoxetine & $N D$ & & $N D$ & $N D$ & $N D$ & $N D$ & & $N D$ & $N D$ & ND \\
\hline sertraline & $N D$ & & $N D$ & $N D$ & $N D$ & $N D$ & & ND & $N D$ & $N D$ \\
\hline
\end{tabular}

$N D$ : less than MDL 
Table 2.6 Concentrations (ng/g) of target compounds detected in fish tissue $(n=7)$ from the freshwater pond

\begin{tabular}{lccccc}
\hline \multicolumn{1}{c}{ Compounds } & Mean & sd & Max & Min & Median \\
\hline lincomycin & $N D$ & & $N D$ & $N D$ & $N D$ \\
trimethoprim & $N D$ & & $N D$ & $N D$ & $N D$ \\
caffeine & 1.3 & 1.6 & 4.5 & $N D$ & 1.2 \\
sulfamethoxazole & $N D$ & & $N D$ & $N D$ & $N D$ \\
diphenhydramine & 0.55 & 0.27 & 0.97 & 0.08 & 0.59 \\
diltiazem & $N D$ & & $N D$ & $N D$ & $N D$ \\
carbamazepine & 0.20 & 0.25 & 0.66 & $N D$ & 0.10 \\
erythromycin & $N D$ & & $N D$ & $N D$ & $N D$ \\
fluoxetine & $N D$ & & $N D$ & $N D$ & $N D$ \\
norfluoxetine & $N D$ & & $N D$ & $N D$ & $N D$ \\
sertraline & $N D$ & & $N D$ & $N D$ & $N D$ \\
& & & & & \\
\hline
\end{tabular}

$N D$ : less than MDL 


\section{CHAPTER 3}

Improving sample throughput and reducing carryover by reprogramming the CTC autosampler for on-line LC-MS/MS analysis using the EQuan system 


\subsection{Introduction}

As the list of pharmaceutically active compounds being reported in various environmental matrices keeps growing, it is obvious that there is a need to develop new analytical techniques that are more effective at analyzing multiple components in a large number of samples in limited time (Balmer et al. 2004; Buser et al. 2006; Mottaleb et al. 2009). Various solid phase extraction (SPE) methods combined with GC-MS and GC-MS/MS (Sacher et al. 2001; Mottaleb et al. 2009; Ramirez et al. 2009), or more recently, LC-MS/MS (Renew and Huang 2004; Lajeunesse et al. 2008; Wang and Gardinali 2012) have been successfully developed to identify and quantify multiple classes of pharmaceuticals in aqueous matrices. The SPE procedures used can be very effective at removing matrix components and concentrating samples to achieve desired sensitivity. However, the preparation requires processing large sample volumes commonly ranging from $200 \mathrm{~mL}$ to $1 \mathrm{~L}$ (Lajeunesse et al. 2008; Wang and Gardinali 2012), which makes it time consuming and laborious.

In order to address the needs for lower detection limits and higher sample throughput, an on-line SPE coupled to mass spectrometry detection was developed to eliminate several preparation steps such as evaporation and reconstitution (Segura 2007; Garcia et al. 2009; Wang and Gardinali 2012). The new protocol was successfully applied to screening and detecting of a variety of environmental contaminants including pharmaceuticals, endocrine disruptors and

groups of pesticides (Segura 2007; Garcia et al. 2009). More recently, on-line 
SPE LC-MS/MS has become a more desired technique in routine analysis of aqueous samples, especially for samples that are time sensitive and need large injection volume.

As shown in Figure 3.1, the time for a typical on-line SPE LC-MS/MS analysis consists of autosampler preparation time, sample withdraw time, SPE sample loading and elution time, and chromatography separation time. On the basis of the fact that loading speed is at $1 \mathrm{~mL} / \mathrm{min}$, total run times could be quite long when large sample volume (i.e. $20-50 \mathrm{~mL}$ ) is used to improve detection limits. To shorten the time between injections, "look ahead" mode can be enabled so that the autosampler sets up for the next injection during the current run. Although the "look ahead" injection mode can be used to incorporate autosampler preparation and sample withdraw time into chromatography time, the sample is still aspired into the sample loop during the next run cycle. In addition, the dead volume of large sample loops $(>5 \mathrm{ml})$ creates a considerable delay of gradient changes and increases the uncertainty of mobile phase mixing.

In this chapter, default autosampler program commands were modified from 1draw sample, 2-move to injection port, 3-aspire sample, 4-inject and 5-washing to 1-inject, 2-wait, 3-switch injection valve to load, 4-washing, 5-draw sample, 6move to injection port, and 7-aspire sample (Figure 3.2). The amount of time that the modified autosampler program took was compared with those needed for the default injection mode and "look ahead" mode. Efficiencies for the washing steps of injection syringe and sample loop which lead to carryover were also 
investigated among the default autosampler program, look ahead, and the modified autosampler program for all the compounds in laboratory routine analysis of water samples.

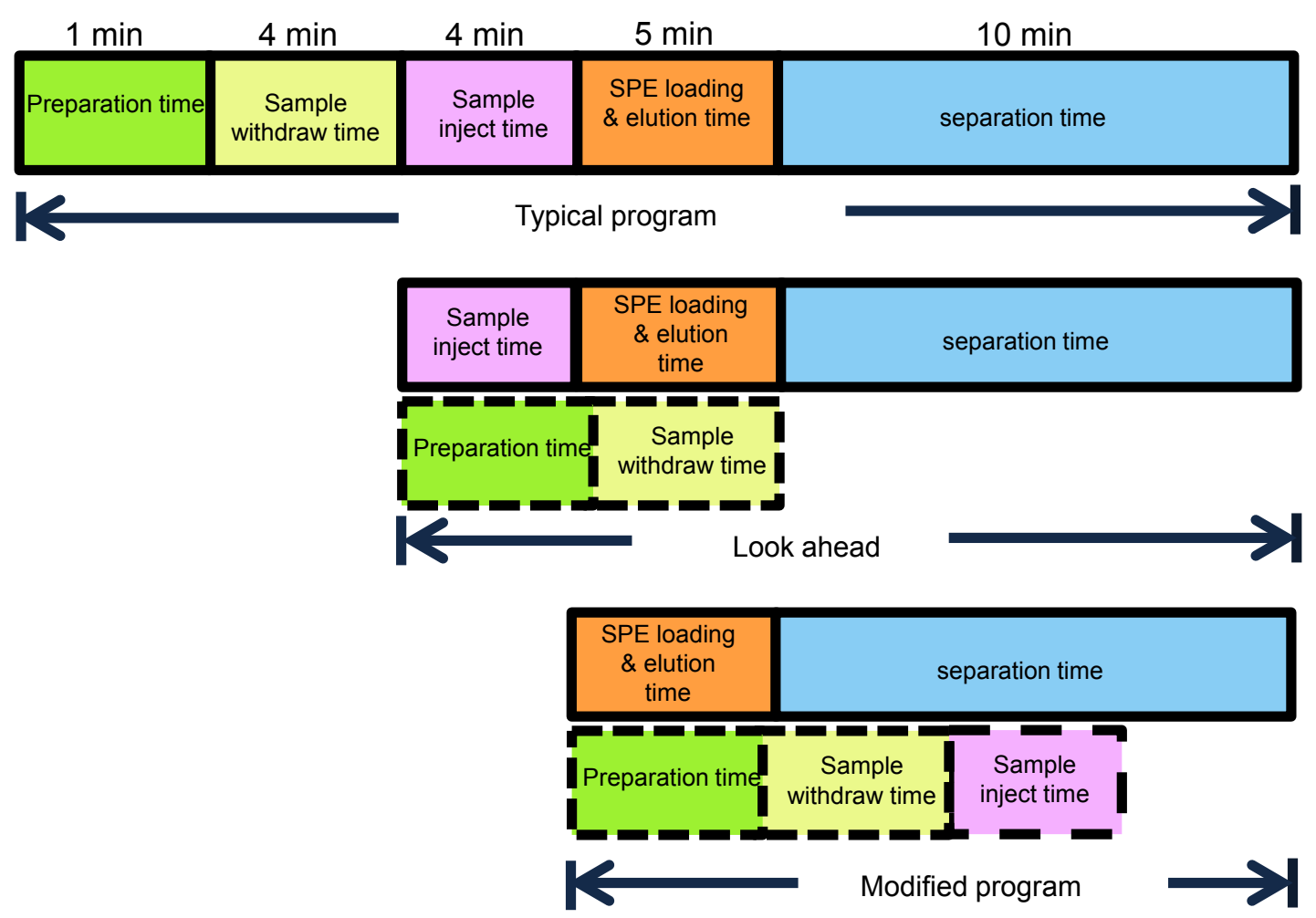

Figure 3.1 Comparison of run times among a typical program, look ahead and the modified program for a $20 \mathrm{~mL}$ on-line EQuan injection with 10 min separation time 
Legend

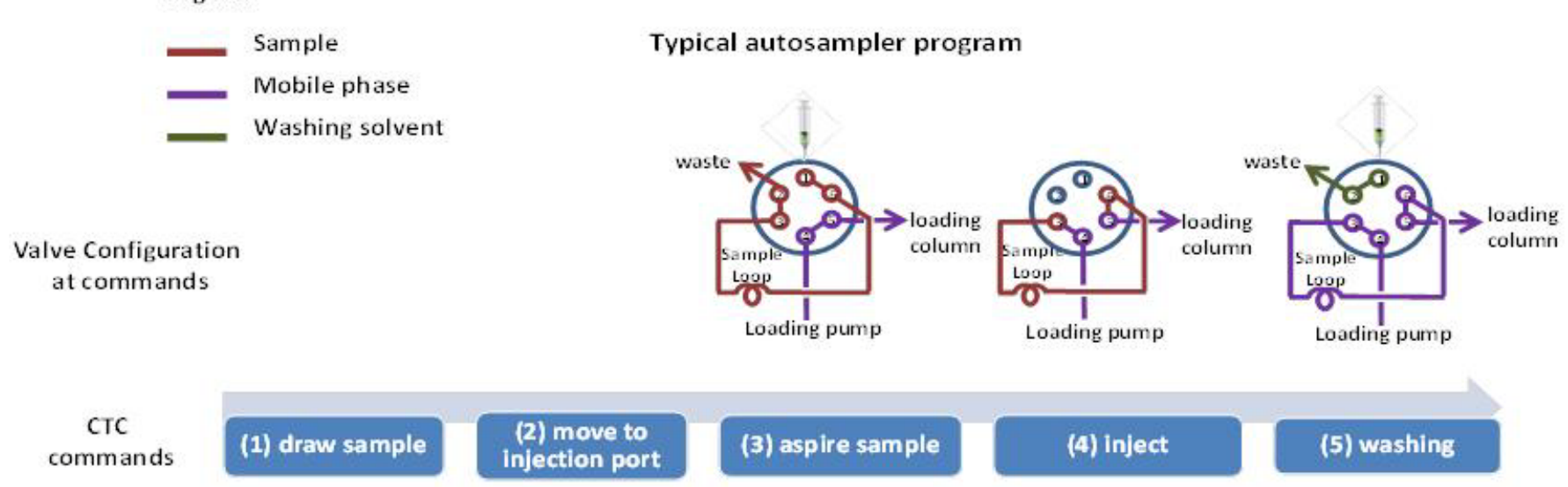

New autosampler program

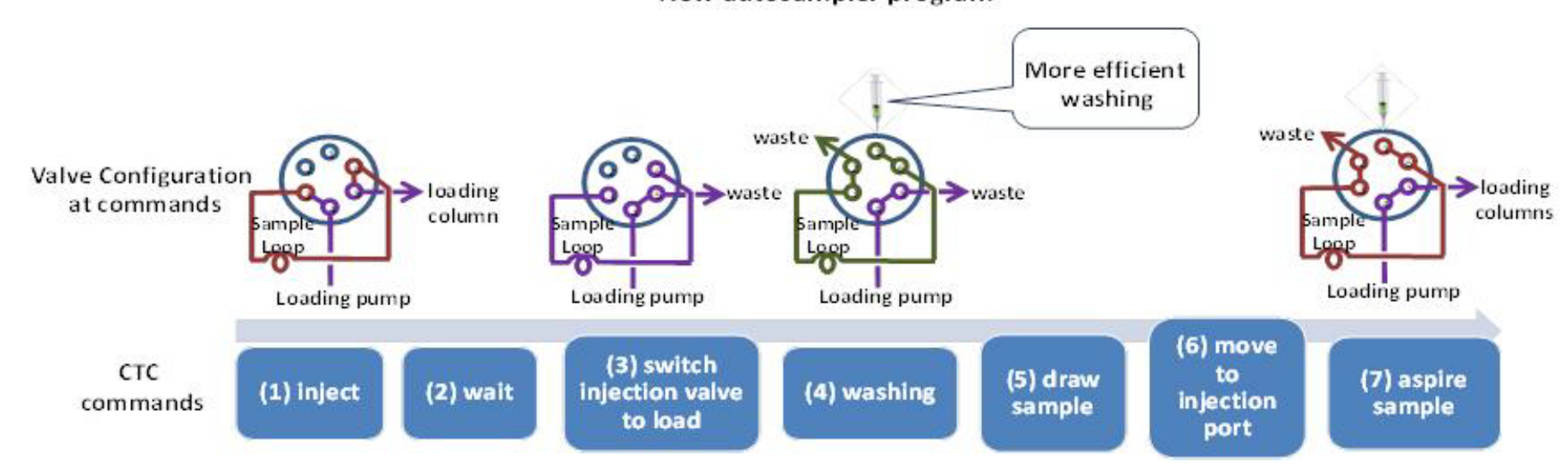

Figure 3.2 Valve configurations and CTC autosampler commands in a typical autosampler program and the new autosampler program 


\subsection{Experimental}

\subsubsection{Reagents, standards and solutions}

The sources and stock solution preparation of all reference standards were the same as those described in Chapter 2. The LC-MS grade methanol, water and formic acid used for mass spectrometry analysis were purchased from Fisher Scientific (Atlanta, GA). The $0.1 \%$ formic acid solution used for mobile phase was prepared daily before analysis.

\subsubsection{Instrumentation}

A Thermo EQuan online SPE system was used in this study. The HPLC system consists of two pumps: Accela 1000 was used as the analytical pump and Accela 600 was used as the loading pump (Thermo Scientific, San Jose, CA). A Hypersil GOLD $20 \mathrm{~mm} \times 2.1 \mathrm{~mm}, 12 \mu \mathrm{m}$ preconcentration column (Thermo Scientific, San Jose, CA) was used as the loading column. The analytical separation was carried out using a Hypersil GOLD column $(150 \mathrm{~mm} \times 3.0 \mathrm{~mm}, 3 \mu \mathrm{m})$. A CTC-PAL autosampler (Thermo Scientific, San Jose, CA) with "Macro editing" function enabled was equipped to perform $5 \mathrm{~mL}$ injections. The autosampler was reprogrammed by rearranging the "commands" in "Macros" which work as the building blocks of the autosampler program. All the samples were analyzed with

a Thermo TSQ Quantum Access triple quadrupole (QqQ) mass spectrometry equipped with a heated electrospray ion source (HESI). Tandem mass 
spectrometry detection was performed in selected reaction monitoring (SRM) mode. Instrument control and data acquisition was performed using the software Xcalibur 2.1 (Thermo Scientific, San Jose, CA).

\subsubsection{Working principle of EQuan system}

The scheme of a typical online SPE setup based on column-switching is shown in Figure 3.3. The working procedure consists of switching a 6-position divert valve on the mass spectrometer which is programmed by the data system to control the loading and elution of the two LC columns. At the "Load" position, 5 $\mathrm{mL}$ of sample was injected into a $5 \mathrm{~mL}$ loop and then loaded onto the SPE column by the loading LC pump (Accela 600), followed by a wash step to remove interferences. The sample was preconcentrated and target compounds were retained on the SPE column while the rest of sample along with matrix was directed to waste. Next, the analytical pump equilibrated the analytical column with starting gradient conditions for the analytical run. Once the sample loading completed, the valve switched to the "Inject" position reversing the solvent flow through the loading column and back flushing analytes onto the analytical column for separation. After a certain amount of time depending upon the gradients, the switching valve returned to the load position to allow the loading column to be reequilibrated. 

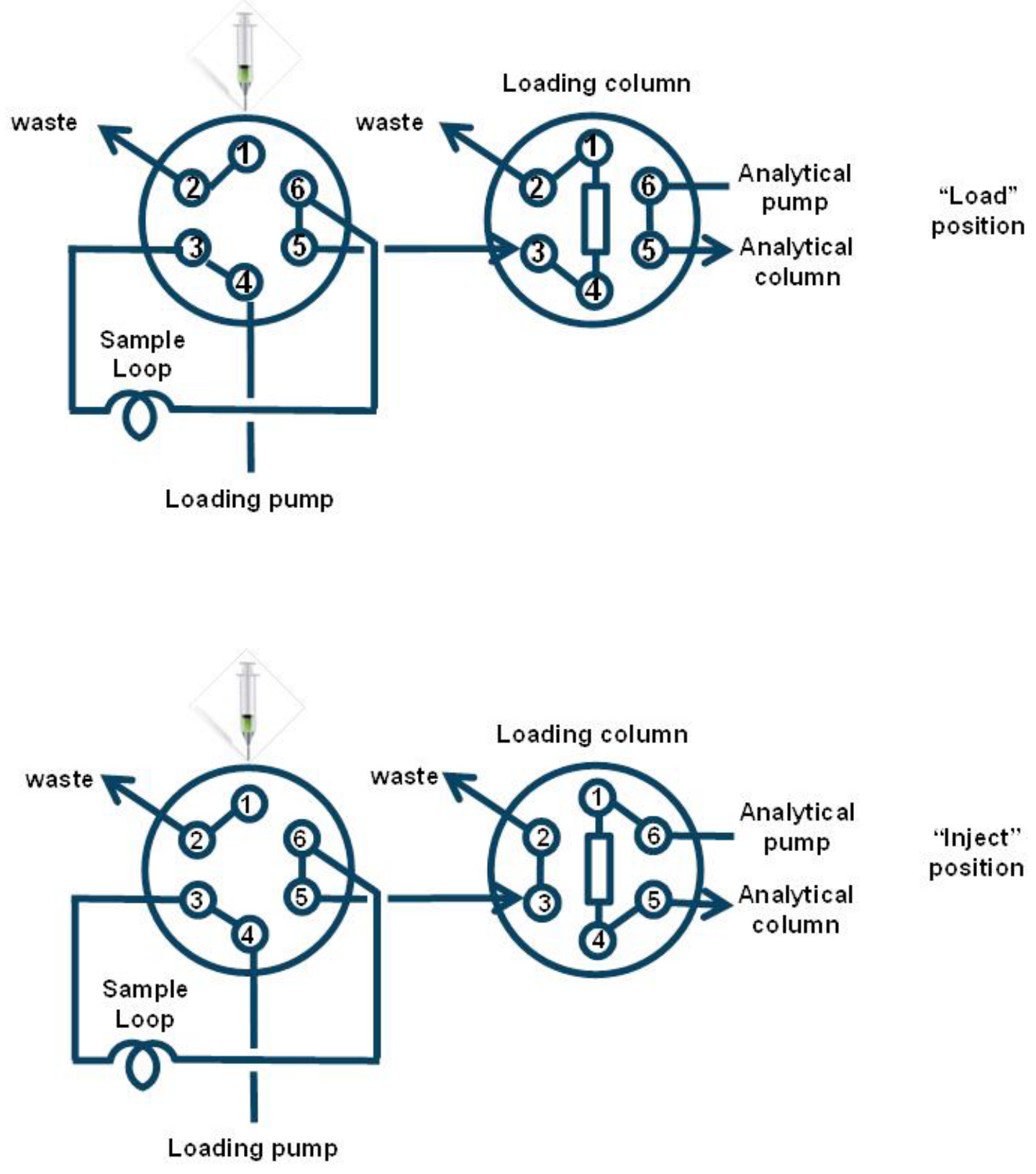

Figure 3.3 Scheme of on-line SPE EQuan system based on column-switching

\subsection{Results and discussions}

Despite having the advantages of improved detection limits and simpler sample preparation steps, on-line EQuan system still suffers from the problems 
associated with extended injection volume if a typical injection program is used. First of all, insufficient washing of the sample loop can lead to carryover. This generally will not become an issue for small volume direct injections as the sample loop (10-50 $\mu \mathrm{L})$ would have been thoroughly flushed many times by the mobile phase passing through it. On the other hand, the EQuan system consists of a sample loop that is so large $(>5 \mathrm{~mL})$ that the sample loop would not be completely flushed before the autosampler draws the next sample, which subsequently leads to carryover. Secondly, unlike the negligible dead time of a direct injection system, one should take into account the dead time that a large sample loop creates because it could be up to several minutes depending on the size of the sample loop and the flow rate of mobile phase. Lastly and most importantly, the total run time could be quite long when an even larger sample volume (e.g., $20 \mathrm{~mL}$ ) is used to improve detection limits. An example of a $20 \mathrm{~mL}$ injection will be discussed below and the run times were compared among a typical autosampler program, look ahead and the modified program.

As shown in Figure 3.1, a typical $20 \mathrm{~mL}$ on-line EQuan injection with a $10 \mathrm{~min}$ of separation time consists of $1 \mathrm{~min}$ of preparation time, 4 min of sample withdraw time, 4 min of sample inject time, and 5 min of SPE loading and elution time. Because this multi-sampling scheme can be quite time consuming, the ability to perform "look-ahead" injections allows for significant time savings. The autosampler will take the subsequent sample in sequence while the mass spectrometer is analyzing the previous sample. Although "look ahead" injections 
can be used to incorporate autosampler preparation and sample withdraw time into chromatography time, a sample is still aspired into the sample loop during the next run cycle. In addition, "look ahead" does not modify the configuration of sample loop, thus the considerable gradient delay and potential carryover are still present.

To address this situation, the current CTC autosampler program was modified from 1-draw sample, 2-move to injection port, 3-aspire sample, 4-inject and 5washing to 1-inject, 2-wait, 3-switch injection valve to load, 4-washing, 5-draw sample, 6-move to injection port, and 7-aspire sample (see Figure 3.2). With this program, the "inject" command simultaneously triggers both data acquisition and injection valve to switch to the inject position. The sample driven by the mobile phase is then loaded onto the loading column. After waiting for a period of time that is long enough for the entire sample to pass through the sample loop, the injection valve is switched back to the load position which shorts the sample loop out of the system. At this point, sample loop can be thoroughly flushed with strong organic solvents such as methanol and acetonitrile resulting a more efficient washing and less carryover. Once the syringe and sample loop have been washed with solvents, the autosampler takes the next sample and aspires it into the sample loop waiting for analysis. In each sequence, the first sample consisting of mobile phase is used to equilibrate the system and to clean the sample loop from previous users. For a 10 min-chromatography separation, up to $34 \%$ run time could be saved compared to the typical program and up to $25 \%$ 
when compared to the already optimized "look ahead" option. A total of $79 \%$ of the selected pharmaceuticals had less carryover when compared to the traditional on-line LC-MS/MS analysis.

\subsection{Conclusions}

The new program incorporates the autosampler preparation time, sample withdraw and aspiration time into the load and chromatography time, resulting in significantly shortened run time for longer sequences. For a $10 \mathrm{~min}-$ chromatography separation, up to $34 \%$ and $24 \%$ of less run time can be saved than the typical program and "look ahead", respectively. The greatly reduced dead volume of the system also prevents the delay of gradient changes and allows for more efficient washing of samples on the loading column before transferring to the analytical column. 


\section{CHAPTER 4}

Uptake and depuration of pharmaceuticals in reclaimed water by mosquito fish (Gambusia holbrooki): A worst case multiple exposure scenario (Jian Wang, Piero Gardinali, Environmental Toxicology and Chemistry, in press) 


\subsection{Introduction}

Human pharmaceuticals and their metabolites enter the environment primarily through wastewater discharge on the basis of the limited removal procedures in traditional wastewater treatment plants (WWTPs) (Daughton and Ternes 1999; Ruhoy and Daughton 2008). Although concentrations in environmental water bodies are typically at $\mathrm{ng} / \mathrm{L}$ to low $\mu \mathrm{g} / \mathrm{L}$ level, these organic pollutants may still pose risks to aquatic species under chronic long-term exposure (Owen et al. 2007; Gunnarsson et al. 2008). For instance, after a 21-day exposure, fluoxetine was shown to induce vitellogenin in male fathead minnows (Pimephales promelas) at $28 \mathrm{ng} / \mathrm{L}$ and venlafaxine caused mortality of fathead minnows at concentration as low as $305 \mathrm{ng} / \mathrm{L}$ (Schultz et al. 2011). Similarly, plasma samples from the rainbow trout (Oncorhynchus mykiss) exposed to sewage effluents were analyzed for 25 pharmaceuticals, of which levonorgestrel was detected in fish plasma at concentrations $(8.5-12 \mathrm{ng} / \mathrm{mL})$ exceeding the human therapeutic plasma level (Fick et al. 2010). Because many aquatic species were shown to have similar physiological receptors to those the pharmaceuticals are originally intended to react with in humans (Owen et al. 2007; Gunnarsson et al. 2008), the data suggested a high risk of pharmacological effects on rainbow trout (Fick et al. 2010). Another study concerning the uptake and depuration as well as bioconcentration factors (BCFs) of pharmaceuticals including moclobemide, 5fluoruracil, carbamazepine, diazepam, carvedilol and fluoxetine was conducted in freshwater shrimp (Gammarus pulex) and water boatman (Notonecta glauca) 
after a 2-day uptake phase (Meredith-Williams et al. 2012). The results showed that BCFs in freshwater shrimp were significantly higher than those found in water boatman, implying that pharmaceuticals may possess even higher risks to smaller aquatic species as environmental pollutants. Recently, I reported that 8 pharmaceuticals including trimethoprim, caffeine, sulfamethoxazole, diphenhydramine, diltiazem, carbamazepine, erythromycin, and fluoxetine were consistently detected in reclaimed water used for daily irrigation at Florida International University Biscayne Bay Campus (North Miami Beach, FL) (Wang and Gardinali 2012; Wang and Gardinali 2012). All these pharmaceuticals are unintentionally released to a freshwater pond through daily irrigation and some of them partition and accumulate in mosquito fish (Gambusia holbrooki) living in this pond (Wang and Gardinali 2012). Therefore, it is expected that aquatic systems like this freshwater pond influenced by substantial inputs of reclaimed water may represent a good model to study the exposure of biological resources to mixtures of pharmaceuticals under relatively natural conditions (Brooks et al. 2006).

In addition, pharmacokinetic data describing the uptake and depuration of pharmaceuticals by aquatic species are extremely limited compared to those in mammals (Paterson and Metcalfe 2008; Meredith-Williams et al. 2012), thus it is important to study the uptake and depuration rates in systems that may represent "worst case exposure" without compromising the environmental relevance (Fick et al. 2010). Most aquatic monitoring studies designed to simulate exposure under environmentally relevant concentrations were performed in artificially clean 
matrices (Nakamura et al. 2008; Paterson and Metcalfe 2008; Hoang et al. 2011; Meredith-Williams et al. 2012), often with exposure concentrations still higher than those would be found under natural conditions by one or more orders of magnitude (Paterson and Metcalfe 2008; Hoang et al. 2011). Although this approach maximizes the chances of quantifying any compounds that might accumulate with confidence, results are often not in good agreement with those from the field (Nakamura et al. 2008; Paterson and Metcalfe 2008; MeredithWilliams et al. 2012). Reclaimed water produced by conventional primary and secondary treatment offers good surrogacy for a worst case exposure scenario and could be used to predict the extent of bioconcentration and toxicological thresholds in aquatic organisms without resourcing to unrealistic concentrations (Paterson and Metcalfe 2008). Moreover, to my knowledge, this is the first study using a non-artificial exposure matrix to assess uptake and depuration kinetics and bioconcentration factors (BCFs) in a highly relevant aquatic species for South Florida protected environments.

\subsection{Materials and methods}

\subsubsection{Reagents, standards and solutions}

LC-MS grade methanol, water and formic acid used for mass spectrometry analysis and SPE extraction, and the Optima grade methylene chloride used for ASE extraction were purchased from Fisher Scientific (Bridgewater, NJ). The $0.1 \%$ formic acid used as mobile phase was prepared daily. The sources for all 43 
pharmaceuticals and surrogate standards along with key physicochemical parameters $\left(\log K_{\mathrm{ow}}\right)$ are shown in Table 4.1. All stock solutions (100 ppm) and working solutions were made in methanol and stored in the dark at $-20^{\circ} \mathrm{C}$.

\subsubsection{Organism husbandry and exposure conditions}

A total of 117 mosquito fish (Gambusia holbrooki) weighing from 0.13 to 1.45 grams in mass and measuring from 2.5 to $5.2 \mathrm{~cm}$ in total length were purchased from Carolina Biological Supply Company (Burlington, NC). Upon arrival, all fish were maintained in two 70-Liter glass aquaria filled with dechlorinated tap water (approximately 60 fish in each aquarium) for 14 days at $26.1 \pm 0.7{ }^{\circ} \mathrm{C}$ under a 14:10h light:dark cycle. All experiments were conducted in accordance with the Institutional Animal Care and Use Committee of Florida International University (\#A3096-01). In order to minimize the variability of concentrations in the reclaimed water and to avoid possible losses on the basis of photo-degradation, a total of 160 liters of reclaimed water were collected from the reclaimed water outlet at Florida International University Biscayne Bay Campus (North Miami, FL) and kept in dark at $16{ }^{\circ} \mathrm{C}$. Water quality parameters including salinity, $\mathrm{pH}$ and dissolved oxygen were monitored throughout the experiments using an YSI 556 MPS Multi Probe System (YSI Incorporated, Yellow Springs, Ohio). 
Table 4.1 Identity, source and physicochemical parameters ( $\log K_{\mathrm{ow}}$ ) for all compounds used in the study

\begin{tabular}{|c|c|c|c|}
\hline & Name & $\log _{K_{\text {ow }}}{ }^{a}$ & Source \\
\hline \multirow{12}{*}{ 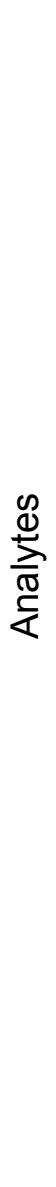 } & Naproxen & 3.10 & Sigma-Aldrich (Milwaukee, WI) \\
\hline & Ibuprofen & 3.79 & Sigma-Aldrich (Milwaukee, WI) \\
\hline & Diclofenac & 4.02 & MP Biomedicals, LLC. (Santa Ana, CA) \\
\hline & Mefenamic acid & 5.28 & Sigma-Aldrich (Milwaukee, WI) \\
\hline & Salicylic acid & 2.24 & Sigma-Aldrich (Milwaukee, WI) \\
\hline & Codeine & 1.28 & Cerilliant Corp. (Round Rock, TX) \\
\hline & Gemfibrozil & 4.77 & Sigma-Aldrich (Milwaukee, WI) \\
\hline & Bezafibrate & 4.25 & Sigma-Aldrich (Milwaukee, WI) \\
\hline & Phenobarbital & 1.33 & Sigma-Aldrich (Milwaukee, WI) \\
\hline & Butalbital & 1.87 & Sigma-Aldrich (Milwaukee, WI) \\
\hline & Fenofibrate & 5.19 & Sigma-Aldrich (Milwaukee, WI) \\
\hline & Atorvastatin & 6.36 & American Radiolabeled Chemicals,Inc. (Saint Louis, MO) \\
\hline
\end{tabular}




\begin{tabular}{|c|c|c|}
\hline Mevastatin & 4.32 & Sigma-Aldrich (Milwaukee, WI) \\
\hline Pravastatin & 3.10 & Sigma-Aldrich (Milwaukee, WI) \\
\hline Fluoxetine & 4.65 & Sigma-Aldrich (Milwaukee, WI) \\
\hline Paroxetine & 3.95 & Sigma-Aldrich (Milwaukee, WI) \\
\hline Sertraline & 5.29 & Sigma-Aldrich (Milwaukee, WI) \\
\hline Diazepam & 2.70 & Cerilliant Corp. (Round Rock, TX) \\
\hline Lorazepam & 2.41 & Cerilliant Corp. (Round Rock, TX) \\
\hline Carbamazepine & 2.25 & Sigma-Aldrich (Milwaukee, WI) \\
\hline Diphenhydramine & 3.11 & Sigma-Aldrich (Milwaukee, WI) \\
\hline Atenolol & -0.03 & Sigma-Aldrich (Milwaukee, WI) \\
\hline Sotalol & -1.89 & Toronto Research Chemicals Inc. (North York, Ontario) \\
\hline Metoprolol & 1.69 & Sigma-Aldrich (Milwaukee, WI) \\
\hline Propranolol & 2.60 & ACROS organics (Morris Plains, NJ) \\
\hline Betaxolol & 2.98 & Sigma-Aldrich (Milwaukee, WI) \\
\hline
\end{tabular}




\begin{tabular}{|c|c|c|}
\hline Carazolol & 2.66 & Toronto Research Chemicals Inc. (North York, Ontario) \\
\hline Pindolol & 1.48 & Sigma-Aldrich (Milwaukee, WI) \\
\hline Nadolol & 1.17 & Sigma-Aldrich (Milwaukee, WI) \\
\hline Salbutamol & 0.64 & Sigma-Aldrich (Milwaukee, WI) \\
\hline Clenbuterol & 2.00 & Sigma-Aldrich (Milwaukee, WI) \\
\hline Enalapril & 2.45 & Sigma-Aldrich (Milwaukee, WI) \\
\hline Diltiazem & 2.79 & Sigma-Aldrich (Milwaukee, WI) \\
\hline Tamoxifen & 6.30 & ACROS organics (Morris Plains, NJ) \\
\hline Metronidazole & -0.02 & ACROS organics (Morris Plains, NJ) \\
\hline Clotrimazole & 6.26 & Sigma-Aldrich (Milwaukee, WI) \\
\hline Glibenclamide & 4.79 & Sigma-Aldrich (Milwaukee, WI) \\
\hline Caffeine & 0.16 & Sigma-Aldrich (Milwaukee, WI) \\
\hline Sulfamethoxazole & 0.48 & Sigma-Aldrich (Milwaukee, WI) \\
\hline Trimethoprim & 0.73 & Sigma-Aldrich (Milwaukee, WI) \\
\hline
\end{tabular}




\begin{tabular}{|c|c|c|c|}
\hline & Lincomycin & 0.29 & Sigma-Aldrich (Milwaukee, WI) \\
\hline & Erythromycin & 2.48 & Sigma-Aldrich (Milwaukee, WI) \\
\hline & Norfluoxetine & 4.07 & Sigma-Aldrich (Milwaukee, WI) \\
\hline & {$\left[{ }^{2} \mathrm{H}_{3}\right]$ Ibuprofen } & N/A & C/D/N Isotopes Inc. (Pointe-Claire, Quebec) \\
\hline & {$\left[{ }^{2} \mathrm{H}_{10}\right]$ Carbamazepine } & N/A & C/D/N Isotopes Inc. (Pointe-Claire, Quebec) \\
\hline & {$\left[{ }^{2} \mathrm{H}_{3},{ }^{13} \mathrm{C}\right]$ Erythromycin } & $\mathrm{N} / \mathrm{A}$ & Toronto Research Chemicals Inc. (North York, Ontario) \\
\hline$\frac{\text { O }}{\frac{0}{0}}$ & {$\left[{ }^{13} \mathrm{C}_{3}\right]$ Caffeine } & $\mathrm{N} / \mathrm{A}$ & Cambridge Isotopes Lab. Inc. (Andover, MA) \\
\hline$\frac{\tilde{\omega}}{\tilde{\omega}}$ & {$\left[{ }^{2} \mathrm{H}_{4}\right]$ Sulfamethoxazole } & N/A & Toronto Research Chemicals Inc. (North York, Ontario) \\
\hline$\stackrel{\bar{\Phi}}{\leftrightarrows}$ & {$\left[{ }^{2} \mathrm{H}_{4}\right]$ Diclofenac } & $\mathrm{N} / \mathrm{A}$ & C/D/N Isotopes Inc. (Pointe-Claire, Quebec) \\
\hline ర్ర & {$\left[{ }^{2} \mathrm{H}_{3}\right]$ Mefenaimic acid } & $\mathrm{N} / \mathrm{A}$ & Toronto Research Chemicals Inc. (North York, Ontario) \\
\hline $\bar{\omega}$ & {$\left[{ }^{2} \mathrm{H}_{4}\right] 2$-hydroxybenzoic acid } & $\mathrm{N} / \mathrm{A}$ & C/D/N Isotopes Inc. (Pointe-Claire, Quebec) \\
\hline & {$\left[{ }^{2} \mathrm{H}_{6}\right]$ Codeine } & $\mathrm{N} / \mathrm{A}$ & Cerilliant Corp. (Round Rock, TX) \\
\hline & {$\left[{ }^{2} \mathrm{H}_{5}\right]$ Atorvastatin } & $\mathrm{N} / \mathrm{A}$ & Toronto Research Chemicals Inc. (North York, Ontario) \\
\hline 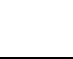 & {$\left[{ }^{2} \mathrm{H}_{3}\right]$ Pravastatin } & $\mathrm{N} / \mathrm{A}$ & Toronto Research Chemicals Inc. (North York, Ontario) \\
\hline
\end{tabular}




$\begin{array}{lll}{\left[{ }^{2} \mathrm{H}_{5}\right] \text { Diazepam }} & \text { N/A } & \text { Cerilliant Corp. (Round Rock, TX) } \\ {\left[{ }^{2} \mathrm{H}_{4}\right] \text { Lorazepam }} & \text { N/A } & \text { Cerilliant Corp. (Round Rock, TX) } \\ {\left[{ }^{2} \mathrm{H}_{7}\right] \text { Atenolol }} & \text { N/A } & \text { C/D/N Isotopes Inc. (Pointe-Claire, Quebec) } \\ {\left[{ }^{2} \mathrm{H}_{3}\right] \text { Albuterol }} & \text { N/A } & \text { C/D/N Isotopes Inc. (Pointe-Claire, Quebec) } \\ {\left[{ }^{2} \mathrm{H}_{5}\right] \text { Enalaprilat }} & \text { N/A } & \text { C/D/N Isotopes Inc. (Pointe-Claire, Quebec) } \\ {\left[{ }^{2} \mathrm{H}_{5}\right] \text { Tamoxefen }} & \text { N/A } & \text { C/D/N Isotopes Inc. (Pointe-Claire, Quebec) } \\ {\left[{ }^{2} \mathrm{H}_{4}\right] \text { Metronidazole }} & \text { N/A } & \text { Toronto Research Chemicals Inc. (North York, Ontario) } \\ {\left[{ }^{2} \mathrm{H}_{5}\right] \text { Clotrimazole }} & \text { N/A } & \text { C/D/N Isotopes Inc. (Pointe-Claire, Quebec) } \\ {\left[{ }^{2} \mathrm{H}_{11}\right] \text { Glyburide }} & \text { N/A } & \text { Toronto Research Chemicals Inc. (North York, Ontario) } \\ {\left[{ }^{2} \mathrm{H}_{6}\right] \text { Fluoxetine }} & \text { N/A } & \text { Cerilliant Corp. (Round Rock, TX) } \\ {\left[{ }^{2} \mathrm{H}_{6}\right] \text { Fenirofibrate }} & \text { N/A } & \text { Toronto Research Chemicals Inc. (North York, Ontario) } \\ {\left[{ }^{2} \mathrm{H}_{5}\right] \text { Timolol }} & \text { N/A } & \text { Toronto Research Chemicals Inc. (North York, Ontario) }\end{array}$

${ }^{a}$ Log $K_{\text {ow }}$ values were calculated from EPI suite V4.0 KOWWIN V1.68 
After the acclimation period, 84 mosquito fish were exposed to reclaimed water in a 70-Liter aquarium as exposure group. The exposure experiments consisted of a 7-day uptake phase with a daily $50 \%$ renewal of the reclaimed water. Approximately eight individual (7-9) mosquito fish and $280 \mathrm{~mL}$ of exposure water were collected for full chemical analysis on $0.2,1,2,3,5$ and 7 days during the uptake phase. After the uptake phase was completed, the remaining fish were transferred to another aquarium filled with clean dechlorinated tap water and allowed to depurate for 14 days. $7-8$ individual fish and $280 \mathrm{~mL}$ of the depuration water were collected on $1,3,6,10$ and 14 day during the depuration phase. Meanwhile, the remaining 33 fish were kept in a separate 70-Liter aquarium filled with clean tap water as control group. 10-13 mosquito fish and $280 \mathrm{~mL}$ of water in the control aquarium were collected on 0 and 7 day of the uptake phase and 14 day of the depuration phase.

Once removed from the aquarium, each fish was rinsed with deionized water to remove any pharmaceutical residue from its surface and euthanized with liquid nitrogen according to the approved IACUC plan. All fish collected at the same sampling event were grouped as one sample and homogenized with a ULTRA TURRAX IKA T18 stainless steel tissuemiser (Wilmington, NC) set to rotate at $10,000 \mathrm{rpm}$. The homogenates were stored at $-20{ }^{\circ} \mathrm{C}$ and thawed at $4{ }^{\circ} \mathrm{C}$ for approximately 10 hours before extraction. All water samples were filtered immediately after collection and stored in dark at or below $4{ }^{\circ} \mathrm{C}$ until they were 
processed. Water extraction was conducted within 7 days of collection to prevent losses on the basis of biodegradation.

\subsubsection{Extraction of fish tissues}

Fish homogenates were extracted using a Dionex ASE 200 accelerated solvent extractor (Dionex Corp., Sunnyvale, CA) fitted with $33 \mathrm{~mL}$ stainless steel extraction cells. 25 grams of $\mathrm{Na}_{2} \mathrm{SO}_{4}$ were placed in the extraction cell containing a glass fiber filter in the outlet side. 1.95-3.16 grams of fish homogenate were placed on top of the $\mathrm{Na}_{2} \mathrm{SO}_{4}$. After adding $200 \mu \mathrm{L}$ of surrogate solution containing $100 \mathrm{ng} / \mathrm{mL}$ of each surrogate standard, the cell was topped with another fiber filter, firmly capped and extracted with methylene chloride. The extraction conditions are as follows: oven temperature $80^{\circ} \mathrm{C}$, pressure $1500 \mathrm{psi}$, heat for $5 \mathrm{~min}$, 1 static cycle, static time $10 \mathrm{~min}$, flush volume $60 \%$ and purge for $120 \mathrm{sec}$. After each extraction cycle, $30 \mathrm{~mL}$ methylene chloride extract was flushed into a $60-\mathrm{mL}$ glass vial and blown down to approximated $5 \mathrm{~mL}$ under gentle nitrogen stream. The concentrated extract was then quantitatively transferred to a $10-\mathrm{mL}$ glass test tube and completely dried under gentle nitrogen stream. The dried extract was reconstituted with $200 \mu \mathrm{L}$ of methanol:0.1\% formic acid 10:90 (v/v). After $10 \mathrm{~min}$ sonication and $1 \mathrm{~min}$ of vortex, samples were transferred and analyzed by LC-MS/MS. 


\subsubsection{Extraction of water samples}

Immediately after collection, water samples were filtered through $0.5 \mu \mathrm{m}$ precombusted glass fiber filters (GE Water \& Process Technologies, Trevose, PA) to remove suspended materials. $200 \mu \mathrm{L}$ of surrogate solution containing $100 \mathrm{ng} / \mathrm{mL}$ of each surrogate standard were added to the samples which were subsequently extracted as described by Wang et al (Wang and Gardinali 2012). Briefly, Oasis HLB (3cc/60mg, Waters Corp., Franklin, MA) solid phase extraction cartridges were conditioned with $3 \mathrm{~mL}$ of methanol followed by $3 \mathrm{~mL}$ of distilled water. After the samples had passed through the HLB cartridges, analytes were eluted with 1 $\mathrm{mL}$ of methanol. Each methanol eluent was evaporated to dryness under a gentle stream of nitrogen gas. Dried residues were reconstituted with $200 \mu \mathrm{L}$ of methanol:0.1\% formic acid 10:90 (v/v). After brief sonication and vortex, the samples were ready for LC-MS/MS analysis.

\subsubsection{LC-MS/MS analysis}

Liquid chromatography was performed on a quaternary Thermo Scientific Accela pump with a Thermo PAL CTC autosampler. A Hypersil GOLD $50 \times 2.1 \mathrm{~mm}, 3$ $\mu \mathrm{m}$ particle size and $175 \AA$ pore size column from Thermo Scientific (Bellefonte, PA) was used for separation. Two sets of mobile phases were used to accommodate target analytes in both positive and negative ionization modes. The mobile phases used for positive ionization mode consisted of $A, 0.1 \%$ formic acid in water (v/v) and B, 100\% methanol. The gradient was (methanol \%): 0 min 
$3 \%, 2 \min 3 \%, 8.5 \min 97 \%, 15 \min 3 \%$ and $17 \min 3 \%$. Acetonitrile and water were used for negative ionization mode. The gradient was (acetonitrile \%): 0 min $30 \%, 1 \min 30 \%, 3 \min 80 \%, 5 \min 30 \%$ and $7 \min 30 \%$. A flow rate of 300 $\mu \mathrm{L} /$ min was maintained for both gradients. The injection volume was set to $10 \mu \mathrm{L}$.

All extracts were analyzed by a Thermo TSQ Quantum Access triple quadrupole (QqQ) mass spectrometry equipped with a heated electrospray ion source (HESI) operated in both positive and negative modes. Tandem mass spectrometry detection was performed in selected reaction monitoring (SRM) mode. Collision energy (CE) and tube lens voltage for each compound were optimized through direct infusion into mass spectrometer at concentration of $1 \mu \mathrm{g} / \mathrm{mL}$ and at the flow rate of $15 \mu \mathrm{L} / \mathrm{min}$. Additional instrumental parameters for the target analytes in positive mode were optimized as follows: Spray voltage at $4000 \mathrm{~V}$, Capillary temperature and Vaporizer temperature at $300{ }^{\circ} \mathrm{C}$, Sheath gas $\left(\mathrm{N}_{2}\right)$ and Aux gas $\left(\mathrm{N}_{2}\right)$ at 30 arbitrary units, lon sweep gas $\left(\mathrm{N}_{2}\right)$ at 0 arbitrary units, and Scan time at 0.02s. Similarly, the parameters in negative mode were set as follows: Spray voltage at $5000 \mathrm{~V}$, Capillary temperature and Vaporizer temperature at $155^{\circ} \mathrm{C}$ and $350{ }^{\circ} \mathrm{C}$, respectively, Sheath gas $\left(\mathrm{N}_{2}\right)$ and Aux gas (N2) at 40 arbitrary units, Ion sweep gas $\left(\mathrm{N}_{2}\right)$ at 0 arbitrary units, and Scan time at $0.05 \mathrm{~s}$.

\subsubsection{QA/QC samples}

Laboratory blanks and spiked blank samples were prepared to evaluate extraction recoveries in both fish and water. Concentrations in water and fish 
samples were determined based on response factors (RFs) of the target analytes relative to the surrogate internal standards. This approach can be used for most trace analysis, as it doesn't require a blank matrix and greatly alleviates the signal suppression or enhancement arising from matrix effects that can affect the sensitivity and response of the mass spectrometer (Wang and Gardinali 2012). Statistically derived method detection limits (MDLs) were used to evaluate the analytical performance in different matrices as they have been well-described and deemed appropiate for environmental analysis instead of signal to noise based limits of detection (LODs) (Ramirez et al. 2007; Ramirez et al. 2009; Wang and Gardinali 2012). Seven MDL replicate samples were prepared as described by Wang et al, (Wang and Gardinali 2012) and extracted as described above. Concentrations below MDLs are reported as "not detected".

\subsubsection{Pharmacokinetics and bioconcentration analysis}

A one-compartment first order kinetics model was used to determine the depuration rate constants $\left(k_{d}\right)$ and corresponding half-lives $\left(t_{1 / 2}\right)$ (Barron et al. 1990). Equation (1)-(3), used to solve $k_{d}$ and $t_{1 / 2}$, are as follows:

$$
\begin{aligned}
& \ln C_{f}=a(t)+b \\
& k_{d}=|a|
\end{aligned}
$$


$t_{1 / 2}=\frac{\ln (2)}{k_{d}}$

Where $C_{f}$ is the concentration in the organism at time "t" during the depuration phase, and the constants $\mathbf{a}$ and $\mathbf{b}$ represent the slope and the intercept of equation (1), respectively.

The uptake rate constant $\left(k_{\mathrm{u}}\right)$ during the exposure phase was determined using the calculated depuration rate constant $\left(k_{d}\right)$ as outlined in equation (4) (Barron et al. 1990):

$C_{f}=\frac{k_{\mathrm{u}}}{k_{\mathrm{d}}} C_{\mathrm{W}}\left(1-\mathrm{e}^{-\mathrm{k}_{\mathrm{d}} \mathrm{t}}\right)$

Where $C_{f}$ is the concentration in the organism at time " $t$ ", and $C_{w}$ is the concentration in water at the same time during the uptake phase.

For comparison, the uptake was also analyzed using the Michaelis-Menten kinetics model (Hoang et al. 2011).

$\frac{1}{C_{\mathrm{f}}}=\left(\frac{\mathrm{K}_{\mathrm{M}}}{\mathrm{C}_{\mathrm{sat}}}\right) \frac{1}{\mathrm{t}}+\left(\frac{1}{\mathrm{C}_{\mathrm{sat}}}\right)$

Where $\mathrm{K}_{\mathrm{M}}$ is the Michaelis-Menten constant, $\mathrm{C}_{\mathrm{f}}$ is the concentration in the fish at time " $\mathrm{t}$ ", and $\mathrm{C}_{\mathrm{sat}}$ is the concentration in the fish at saturated state (maximal 
concentration). $K_{M} / C_{\text {sat }}$ and $1 / C_{\text {sat }}$ are the slope and the intercept of equation (5), respectively. $C_{\text {sat }}$ can be determined by plotting $1 / C_{f}$ over $1 /$ t.

Bioconcentration factors were evaluated by three different approaches. The steady-state bioconcentration factor was calculated using the concentration measure in the fish at $7 \mathrm{~d}$ of exposure $\left(\mathrm{C}_{\mathrm{f}}\right)$ and the averaged water concentration $\left(C_{w}\right)$ from all points taken during the uptake phase.

$\mathrm{BCF}_{\mathrm{a}}=\frac{\mathrm{C}_{\mathrm{f}}}{\mathrm{C}_{\mathrm{w}}}$

The ratio of the uptake and depuration rate constants was calculated as the second bioconcentration factor.

$\mathrm{BCF}_{\mathrm{b}}=\frac{\mathrm{k}_{\mathrm{u}}}{\mathrm{k}_{\mathrm{d}}}$

Lastly, a third value for the bioconcentration factor was calculated using $\mathrm{C}_{\text {sat }}$ as derived from equation (5) using the averaged concentrations in water during the whole exposure time.

$\mathrm{BCF}_{\mathrm{C}}=\frac{\mathrm{C}_{\mathrm{sat}}}{\mathrm{C}_{\mathrm{w}}}$

\subsection{Results and Discussions}

No significant changes in water quality parameters or fish mortalities were observed throughout the study. Temperature was maintained at $26.1 \pm 0.7{ }^{\circ} \mathrm{C}$, 
dissolved oxygen was at $5.5 \pm 0.6 \mathrm{mg} / \mathrm{L}$, and $\mathrm{pH}$ was at $8.6 \pm 0.2$. No free chlorine was detected in any of the water samples.

As shown in Table 4.2, a total of 26 pharmaceuticals ranging from $21.3 \mathrm{ng} / \mathrm{L}$ for fluoxetine to $8638 \mathrm{ng} / \mathrm{L}$ for caffeine were detected in the reclaimed water used in the exposure experiment. The relative standard deviation for exposure concentrations was less than $30.4 \%$ in most cases, with exceptions for sulfamethoxazole at $48.2 \%$, and for naproxen at $52.4 \%$, respectively. No target analytes were detected in control water or control fish at levels above the corresponding MDLs.

\section{Table 4.2 Pharmaceuticals detected in exposure reclaimed water}

\begin{tabular}{cccc}
\hline $\begin{array}{c}\text { Ionization } \\
\text { mode }\end{array}$ & Compound & $\begin{array}{c}\text { Concentration in } \\
\text { reclaimed water } \\
(\mathrm{ng} / \mathrm{L})\end{array}$ & $\mathrm{RSD}(\%)$ \\
\hline Positive & Metronidazole & $66.3 \pm 11.1$ & 16.8 \\
Positive & Sotalol & $594 \pm 23.1$ & 3.9 \\
Positive & Salbutamol & $68.8 \pm 11.3$ & 16.5 \\
Positive & Atenolol & $4605 \pm 405$ & 8.8 \\
Positive & Codeine & $201 \pm 35.6$ & 17.7 \\
Positive & Trimethoprim & $816 \pm 109$ & 13.3
\end{tabular}




\begin{tabular}{|c|c|c|c|}
\hline Positive & Caffeine & $8638 \pm 937$ & 10.9 \\
\hline Positive & Nadolol & $161 \pm 35.3$ & 22.0 \\
\hline Positive & Sulfamethoxazole & $1152 \pm 556$ & 48.2 \\
\hline Positive & Metoprolol & $196 \pm 21.1$ & 10.7 \\
\hline Positive & Propranolol & $148 \pm 33.2$ & 22.4 \\
\hline Positive & Betaxolol & $523 \pm 34.1$ & 6.5 \\
\hline Positive & Diphenhydramine & $5218 \pm 1350$ & 25.9 \\
\hline Positive & Diltiazem & $144 \pm 43.7$ & 30.4 \\
\hline Positive & Carbamazepine & $1229 \pm 112$ & 9.1 \\
\hline Positive & Erythromycin & $288 \pm 45.6$ & 15.8 \\
\hline Positive & Fluoxetine & $21.3 \pm 3.29$ & 15.5 \\
\hline Positive & Lorazepam & $117 \pm 13.4$ & 11.4 \\
\hline Positive & Tamoxifen & $33.5 \pm 1.6$ & 4.7 \\
\hline Positive & Glibenclamide & $115 \pm 14.8$ & 12.8 \\
\hline Negative & Phenobarbital & $55.7 \pm 12.5$ & 22.5 \\
\hline Negative & Butalbital & $126 \pm 18.5$ & 14.7 \\
\hline Negative & Naproxen & $73.0 \pm 38.2$ & 52.4 \\
\hline
\end{tabular}




\begin{tabular}{llcc} 
Negative & Diclofenac & $958 \pm 106$ & 11.1 \\
Negative & Ibuprofen & $331 \pm 35.8$ & 10.8 \\
Negative & Gemfibrozil & $2272 \pm 605$ & 26.6 \\
\hline
\end{tabular}

The observed concentrations for trimethoprim, caffeine, sulfamethoxazole, diphenhydramine, diltiazem, carbamazepine, erythromycin, and fluoxetine in reclaimed water were higher than those in previous reports (Wang and Gardinali 2012; Wang and Gardinali 2012), in which reclaimed water was directly drawn from irrigational sprinklers. The mechanism for the loss in the reclaimed water drawn from sprinklers is unclear but is likely the results of different input sources. Therefore, the reclaimed water collected directly from the source outlet with higher concentrations was used here in order to maximize the chances of detecting more compounds that might be bioconcentrated by fish. Among the 26 pharmaceuticals detected in reclaimed water, 5 pharmaceuticals including caffeine, diphenhydramine, diltiazem, carbamazepine, and ibuprofen were observed in mosquito fish as early as $5 \mathrm{~h}$ from the start of the exposure to reclaimed water. Uptake and depuration rate constants along with BCFs are summarized in Table 4.3 and shown in Figure 4.1. One-compartment first order and Michaelis-Menten kinetics models were fitted to the uptake and depuration curves. In general, both models fitted all uptake curves fairly well, while Michaelis-Menten kinetics model fitted the uptake curves better for caffeine $\left(R^{2}=0.9980\right)$, diphenhydramine $\left(R^{2}=0.9521\right)$, and carbamazepine $\left(R^{2}=0.9849\right)$, 
whereas one-compartment first order model fitted the uptake curve better for diltiazem $\left(R^{2}=0.9871\right)$ and ibuprofen $\left(R^{2}=0.9812\right)$. All depuration curves appeared to follow first order elimination.

Table 4.3 Uptake and depuration rate constants, half-lives and BCFs for selected pharmaceuticals in mosquito fish (Gambusia holbrooki)

\begin{tabular}{lllllll} 
Compound & $\mathrm{k}_{\mathrm{u}}\left(\mathrm{mL} \mathrm{g} \mathrm{g}^{-1}\right)$ & $\mathrm{k}_{\mathrm{d}}\left(\mathrm{h}^{-1}\right)$ & $\mathrm{t}_{1 / 2}(\mathrm{~h})$ & $\mathrm{BCF}_{\mathrm{a}}$ & $\mathrm{BCF}_{\mathrm{b}}$ & $\mathrm{BCF}_{\mathrm{c}}$ \\
\hline Caffeine & 0.0011 & 0.0049 & 141 & 2.0 & 0.23 & 2.0 \\
Diphenhydramine & 0.21 & 0.020 & 34 & 16 & 10 & 15 \\
Diltiazem & 0.033 & 0.0059 & 117 & 16 & 5.6 & 16 \\
Carbamazepine & 0.0085 & 0.0098 & 71 & 1.4 & 0.87 & 1.4 \\
Ibuprofen & 0.64 & 0.022 & 32 & 28 & 29 & 27 \\
\hline
\end{tabular}

\subsubsection{Uptake and depuration kinetics}

The uptake of caffeine by mosquito fish was very rapid as the internal concentration reached the plateau and remained approximately constant after 5 $\mathrm{h}$ of the exposure. Similar uptake behavior was also documented in Nile tilapia (Oreochromis niloticus) (Gomez-Martinez 2011). However, the depuration rate of caffeine in mosquito fish was much slower than that found in Nile tilapia, in which caffeine had a half-live of $4.95 \mathrm{~h}$ (Gomez-Martinez 2011) compared to the $141 \mathrm{~h}$ 
for mosquito fish calculated in this study. The results indicate that caffeine would be uptaken by mosquito fish and Nile tilapia at relatively the same rate but remain in mosquito fish for a longer time and therefore, pose higher risks to mosquito fish than it does to Nile tilapia. For carbamazepine, the uptake rate constant $\left(0.0085 \mathrm{~mL} \mathrm{~g}^{-1} \mathrm{~h}^{-1}\right.$ or $0.20 \mathrm{~L} \mathrm{~kg}^{-1}$ day $\left.^{-1}\right)$ and depuration rate constant (0.0098 $\mathrm{mL} \mathrm{g}^{-1} \mathrm{~h}^{-1}$ or $0.24 \mathrm{~L} \mathrm{~kg}^{-1}$ day $\left.^{-1}\right)$ in mosquito fish were similar to those reported in water boatman (Notonecta glauca) which were calculated at $0.29 \mathrm{~L}$ $\mathrm{kg}^{-1}$ day $^{-1}$ and $1.2 \mathrm{~L} \mathrm{~kg}^{-1} \mathrm{day}^{-1}$, respectively (Meredith-Williams et al. 2012). As a result, the rate constant-based $\mathrm{BCF}$ of 0.87 in mosquito fish was slightly higher than that of 0.24 in water boatman (Meredith-Williams et al. 2012). In contrast, a higher BCF for carbamazepine in freshwater shrimp (Gammarus pulex) was calculated at 7.1 in the same study (Meredith-Williams et al. 2012), and the value was 30 times and 8 times higher than that in water boatman and mosquito fish, respectively. The phenomenon that BCFs are organism size related has been previously discussed (Hendriks et al. 2001). The findings by Meredith-Williams et al. (Meredith-Williams et al. 2012) indicated that BCFs were indeed reduced as the organism size increased. Among the 5 pharmaceuticals detected in mosquito fish, diphenhydramine had the highest internal concentration of $82 \mathrm{ng} / \mathrm{g}$ that was reached after $77 \mathrm{~h}$ of the exposure to reclaimed water. During the depuration, diphenhydramine was quickly eliminated with the second shortest half live of $34 \mathrm{~h}$. Similarly, a gradual uptake followed by a rapid depuration was observed for ibuprofen resulting the shortest half live $(32 \mathrm{~h})$ and the highest BCF among the five pharmaceuticals. Diltiazem followed a similar uptake curve as observed for 
carbamazepine but the half-life $(117 \mathrm{~h})$ was longer than that of carbamazepine (71 h). Published data on the pharmacokinetics of diphenhydramine, diltiazem, and ibuprofen in aquatic organisms are limited, but data are available on BCFs of these compounds which will be compared below.

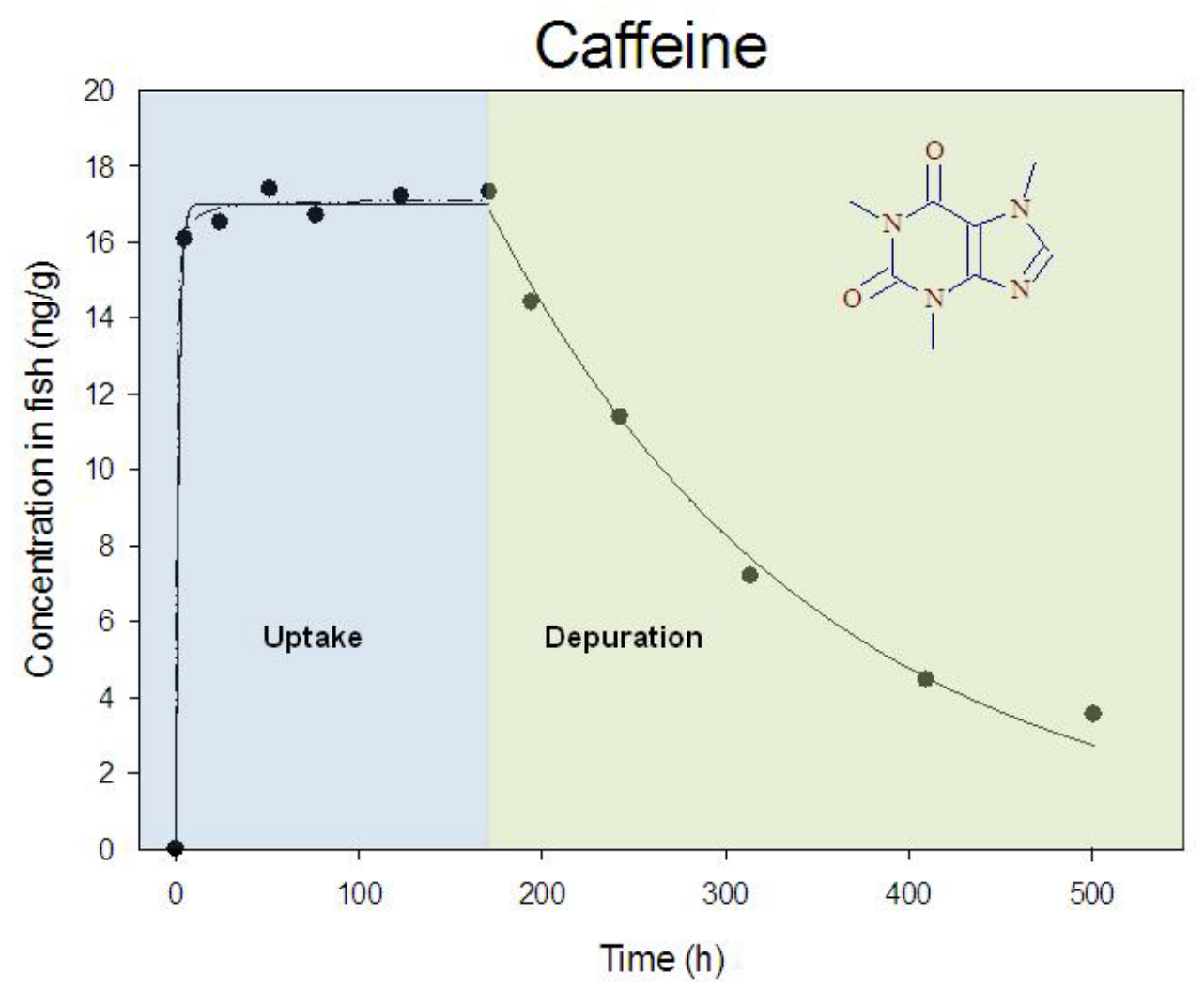



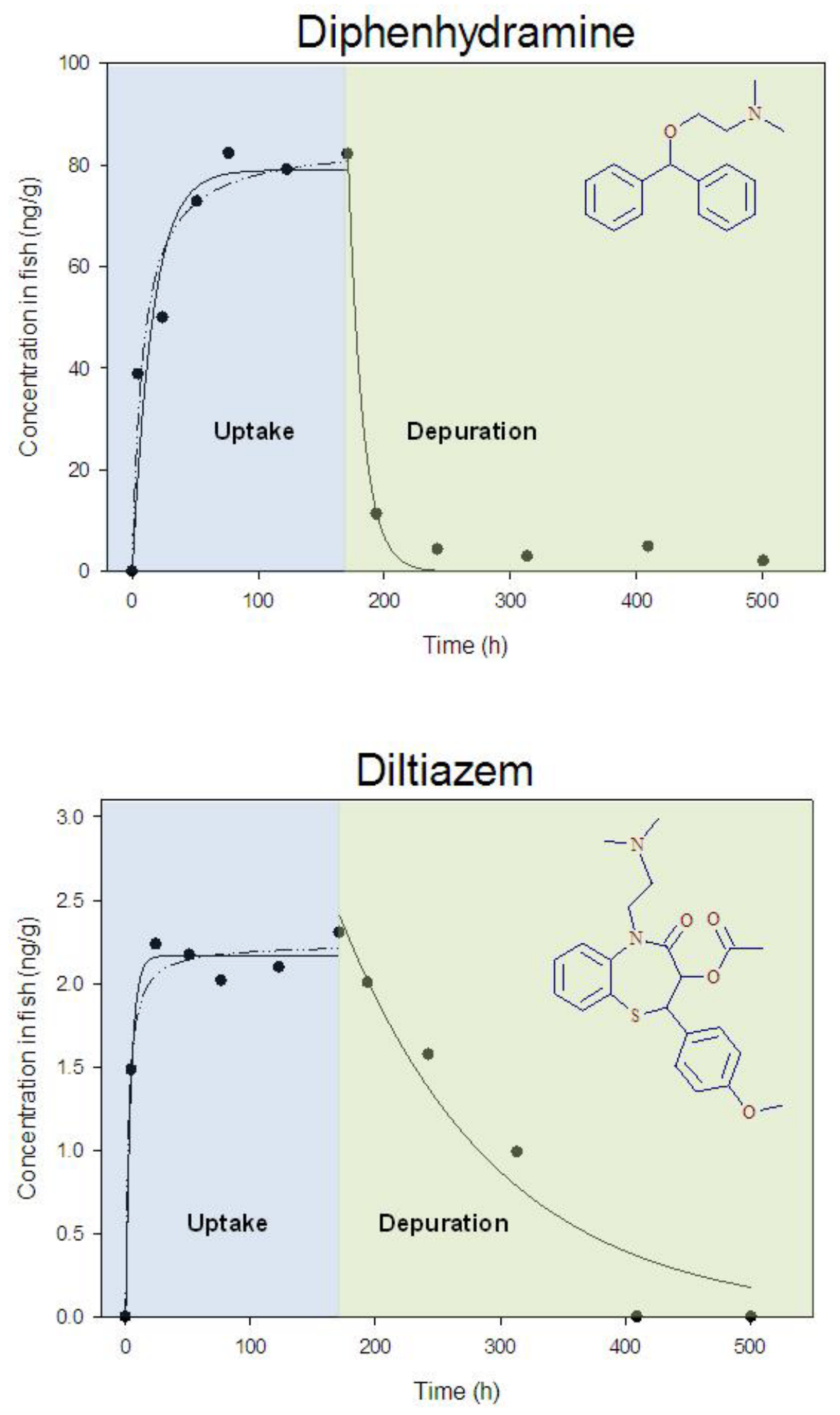

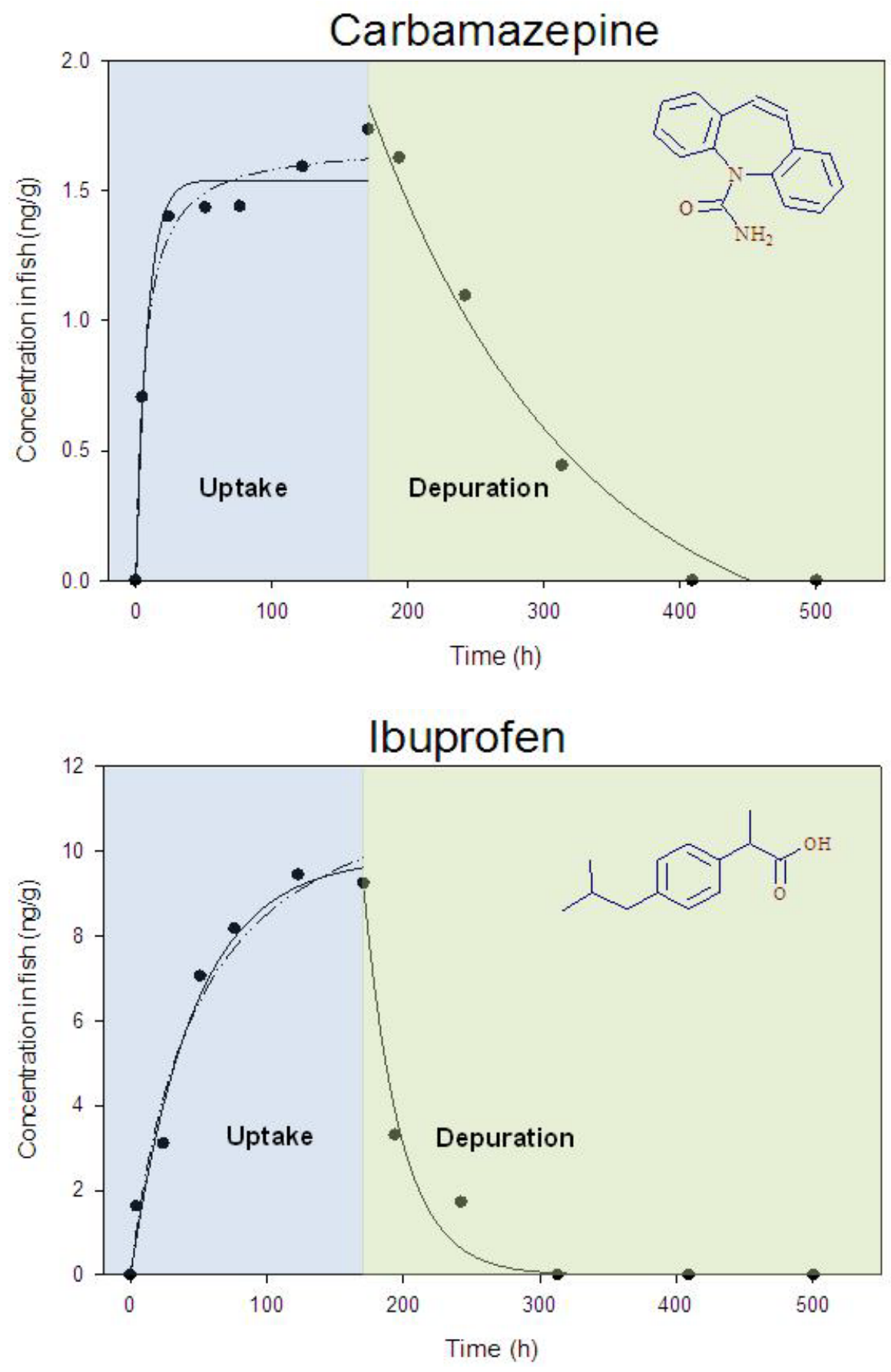

Figure 4.1 Uptake and depuration curves for mosquito fish (Gambusia holbrooki) exposed to reclaimed water: the dotted lines represent for the Michaelis-Menten curve fit and the solid lines represent for the first order curve fit 


\subsubsection{Bioconcentration factors}

In Table 4.3, $\mathrm{BCF}_{\mathrm{b}}$ ranged from 0.23 to 29 and increased in the order of caffeine $<$ carbamazepine $<$ diltiazem $<$ diphenhydramine $<$ Ibuprofen. $\mathrm{BCF}_{\mathrm{a}}$ and $\mathrm{BCF}_{\mathrm{c}}$ ranged from 2.0 to 28 , and increased in the order of carbamazepine < caffeine < diltiazem < diphenhydramine < Ibuprofen. BCFs found for caffeine, diphenhydramine, and carbamazepine in mosquito fish were respectively lower than those reported from the previous study for natural exposure in the pond influenced by irrigation with reclaimed water (29 for caffeine, 821 for diphenhydramine, and 108 for carbamazepine) (Wang and Gardinali 2012). The lower BCFs in the laboratory experiment with full strength reclaimed water could possibly be related to the availability of additional dietary routes for the mosquito fish in natural settings (Blanco et al. 2004). In addition, the pond system may contain higher concentration of dissolved organic carbon (DOC) that has been shown to significantly enhance the BCFs of organic pollutants (Pickhardt et al. 2006). Despite the known effects of acid base equilibrium on BCFs for ionizable compounds (Nakamura et al. 2008), the $\mathrm{pH}$ was not believed to have significant effects on the BCFs in this study because the $\mathrm{pH}$ of 8.6 in reclaimed water was similar to that in the fresh water pond (Wang and Gardinali 2012). BCFs calculated for carbamazepine, diltiazem, and ibuprofen in this study were in reasonably good agreement (within one order of magnitude) with plasma BCFs reported in the rainbow trout (Oncorhynchus mykiss) after exposed to treated sewage effluents (Fick et al. 2010). BCFs for carbamazepine in mosquito fish 
(ranging from 0.87 to 1.4 ) were also in agreement with tissue BCF of 0.52 in rainbow trout (Zhang et al. 2010). Ibuprofen however, appeared to bioconcentrate less in rainbow trout (BCF of 1.5) (Zhang et al. 2010) than it did in mosquito fish (BCF of 29) in this study. Upon reviewing the literature (Brooks et al. 2005; Chu and Metcalfe 2007; Nakamura et al. 2008; Paterson and Metcalfe 2008), fluoxetine, one of the most widely found pharmaceuticals in fish was not detected in any of the fish samples even though it was detected in the exposure reclaimed water at a concentration of $21.3 \pm 3.29 \mathrm{ng} / \mathrm{L}$ in this study. This is not surprising because the concentration of fluoxetine in fish would be slightly above the MDL of $1.2 \mathrm{ng} / \mathrm{g}$ (Wang and Gardinali 2012) even if it bioconcentrated based on the highest BCF value reported at 80 (Paterson and Metcalfe 2008).

Lastly, when constructing graphs (Figure 4.2) of calculated BCFs vs Log $K_{\text {ow, }}$ excellent positive relationships were observed for all compounds, with the exception of caffeine that had to be excluded from the plots. This behavior is consistent with previously proposed predictive models (Gossett et al. 1983). The abnormal behavior of caffeine was probably on the basis of its extreme Log $K_{\mathrm{ow}}$ value of -0.07 (Stackelberg et al. 2007), thus caffeine most likely reached equilibrium between water and fish tissue instead of bioconcentration. 

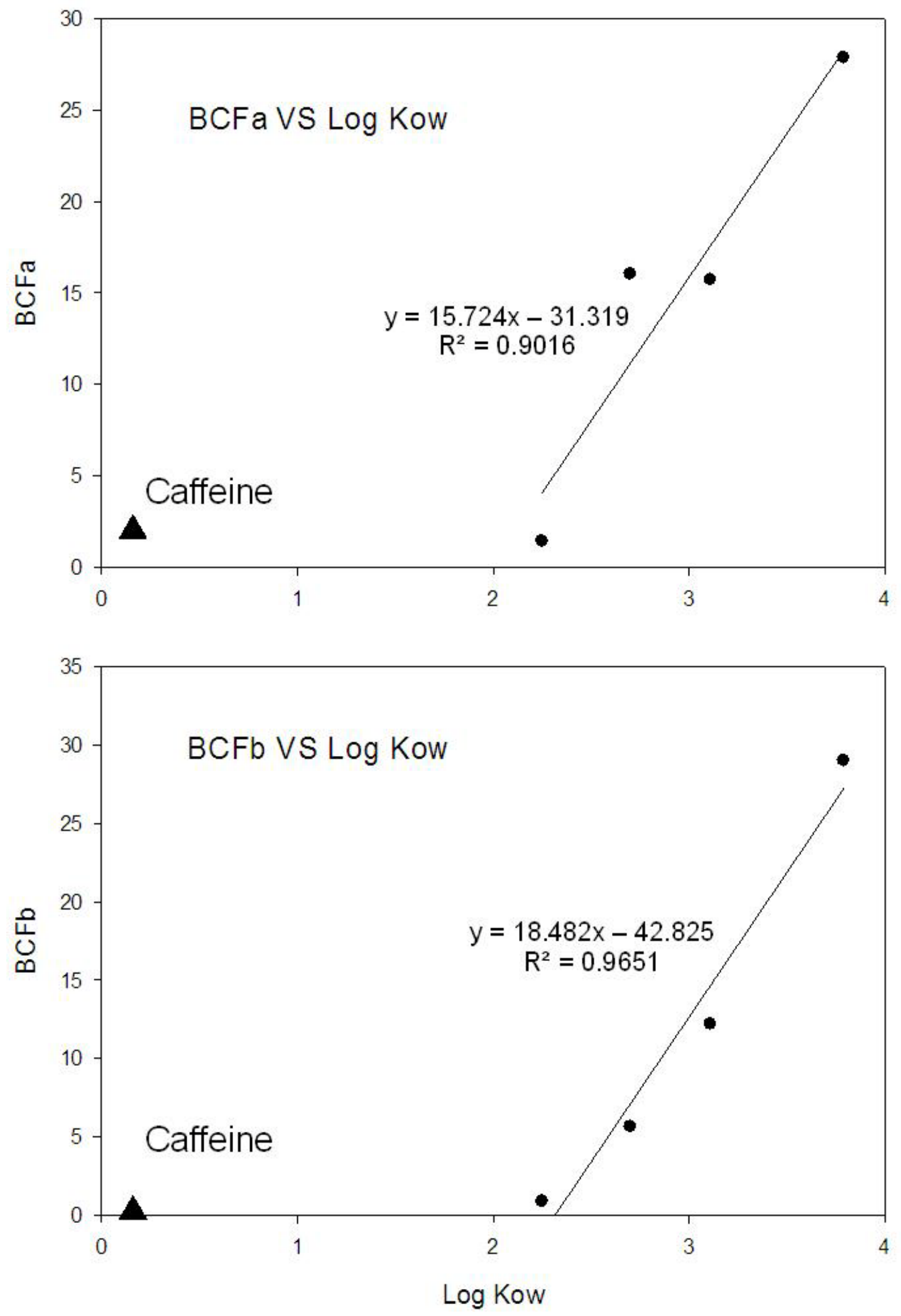


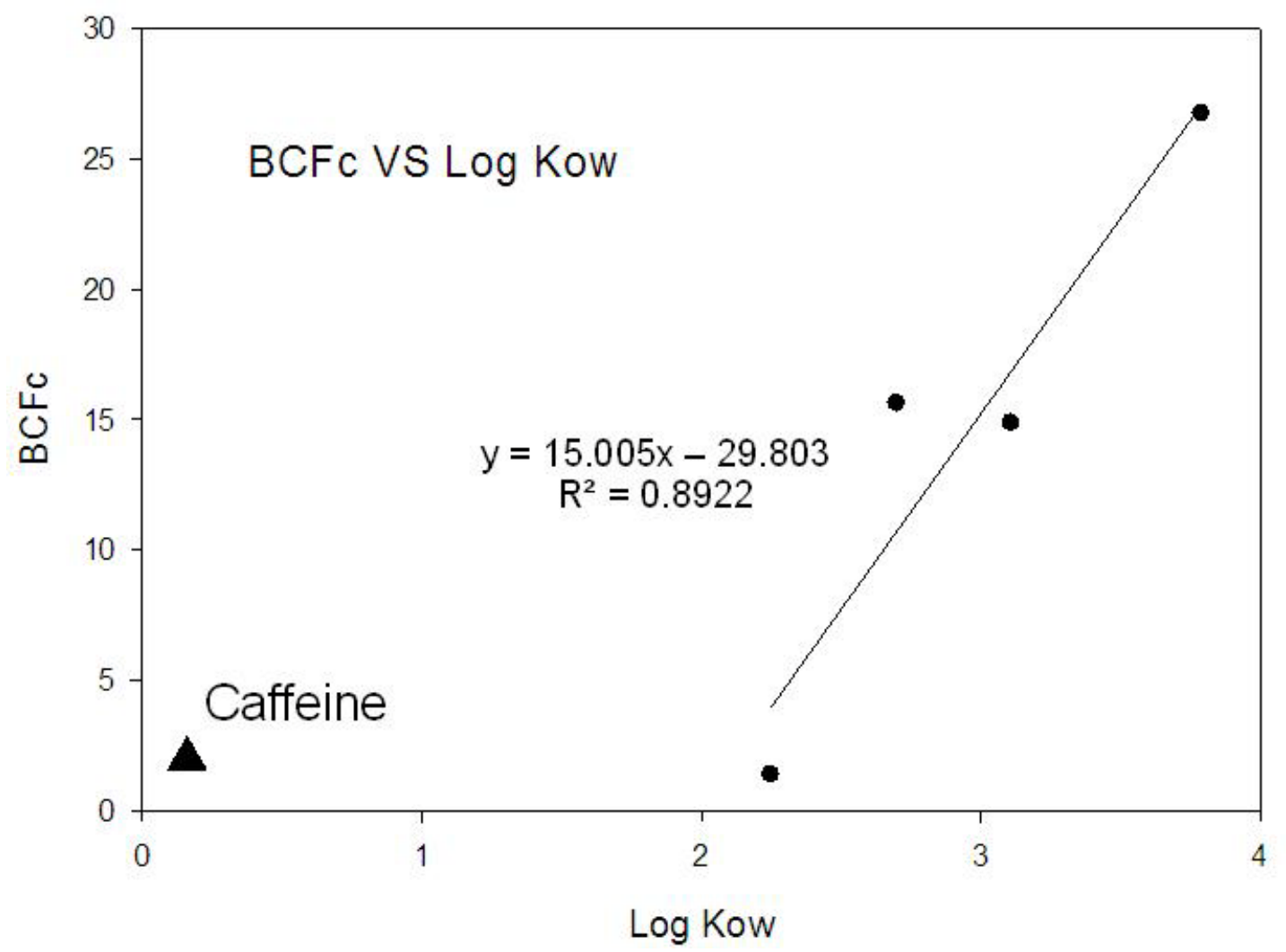

Figure 4.2 Correlations between bioconcentration factors and $\log K_{\mathrm{ow}}\left(\mathrm{BCF}_{\mathrm{a}}\right.$ : based on steady state concentrations, $\mathrm{BCF}_{\mathrm{b}}$ : based on rate constants, and $\mathrm{BCF}_{\mathrm{c}}$ : based on saturation state concentrations)

\subsection{Conclusions}

This study described the pharmacokinetics and bioconcentration of 5 pharmaceuticals in mosquito fish under the "worst case" exposure scenario. The results showed that some pharmaceuticals such as diphenhydramine could be accumulated in fish up to $82 \mathrm{ng} / \mathrm{g}$ while some pharmaceuticals such as caffeine 
will rather equilibrate with the surrounding waters and would persist for a period of several days. The study also showed that a number of other compounds detected in rather high concentrations (e.g. diclofenac and gemfibrozil) did not accumulate in fish despite having moderately high Log $K_{\text {ow }}$ values implying that other mechanisms may have played a role in keeping them in solution. Because many aquatic species have similar physiological receptors to those originally targeted for pharmacological effects in humans (Owen et al. 2007; Gunnarsson et al. 2008), non-target species under chronic exposure may show potential adverse effects (Berninger and Brooks 2010; Fick et al. 2010). Therefore, future studies focused on biotransformation and metabolism in fish particularly targeting compounds with long persistence would provide useful information for predicting toxic effects in fish. 


\section{CHAPTER 5}

Identification of phase II pharmaceutical metabolites in reclaimed water using high resolution benchtop Orbitrap mass spectrometry

(Manuscript ready for submission to Analytical Chemistry, 2013) 


\subsection{Introduction}

Pharmaceutically active compounds are now well-acknowledged pollutants in the environment, especially in surface water and wastewater. They are released into the environment largely through the discharge of wastewater treatment plants (WWTPs) as a result of excretion and metabolism by humans and animals, and additionally disposal of unused or expired drugs (Daughton and Ternes 1999; Miao and Metcalfe 2003; Vanderford et al. 2003). Many studies have demonstrated that pharmaceuticals may pose adverse effects to wildlife and humans (drinking water) under chronic long-term exposure despite the relatively low concentrations in the environment (Vanderford et al. 2003; Brooks et al. 2005). Therefore, it is not surprising that much attention regarding pharmaceuticals in the environment has been drawn on the basis of ecological concerns and public health.

The presence of pharmaceutically active compounds in the environment is assessed largely by measuring the parent drugs (i.e., carbamazepine and fluoxetine) and to a lesser extent transformation products (i.e., 10,11-dihydro10,11-dihydroxycarbamazepine, the major metabolite of carbamazepine, and norfluoxetine, the major metabolite of fluoxetine) (Miao and Metcalfe 2003; Brooks et al. 2005; Miao et al. 2005; Chu and Metcalfe 2007). Parent drugs can be excreted as the unchanged form (Miao et al. 2005) whereas transformation products can be formed as metabolites by undergoing chemical or biochemical transformations (Gobel et al. 2004) or as photodegradation products by exposure 
to sunlight (Bonvin et al. 2012). Parent drugs can undergo a broad range of reactions during metabolism which can be categorized into two phases (Murphy 2001). Phase I reactions including oxidation, reduction and hydrolysis convert the parent drug into a more polar metabolite by adding or revealing functional groups such as $-\mathrm{OH},-\mathrm{SH},-\mathrm{NH}_{2}$ and $-\mathrm{COOH}$, etc.. The mechanism involves a variety of enzymes such as cytochrome P450 oxidase and NADPH-cytochrome P450 reductase introducing reactive and polar groups into their substrates (Lu et al. 1969; Guengerich and Johnson 1997; Danielson 2002; Gu et al. 2003). The conversions including benzene to phenol (Schultzen and Naunyn 1867) and benzaldehyde to benzoic acid (Wohler and Frerichs 1848) were among the very first pieces of evidence indicating the ability of the body to oxidize xenobiotic compounds. Phase II metabolism is a conjugation reaction catalyzed by a large group of broad-specificity transferases (Williams 1947). Parent drugs and active metabolites are often conjugated with charged species such as glutathione (GSH), sulfate, glycine, or glucuronic acid to be detoxified (Chen et al. 2005; Berthiller et al. 2006; Holcapek et al. 2008). Current research is focusing heavily on unchanged parent drugs (Celiz et al. 2009). However, it is important to realize that the exposure to metabolites may have hazardous effects similar to those of the parent drugs (Bedner and MacCrehan 2006).

Numerous analytical instruments have been applied to investigate the occurrence of parent drugs in a variety of environmental matrices (Vanderford et al. 2003; Davis et al. 2006; Kwon and Armbrust 2006; Ramirez et al. 2007; 
Schultz et al. 2010; Wang and Gardinali 2012). Liquid chromatography coupled with triple quadrupole $(\mathrm{QqQ})$ and to a lesser extend ion trap mass spectrometry is undoubtedly the most used approach in such targeted analysis. With the aids of high sensitivity and selectivity that tandem mass spectrometry offers when encountering complex matrices, most parent drugs can be detected and quantified at lower ng/L or ppt level (Miao and Metcalfe 2003; Miao et al. 2005; Lajeunesse et al. 2008; Wang and Gardinali 2012). In contrast, little attention has been paid to the identification, let alone quantification, of pharmaceutical metabolites (Celiz et al. 2009) because there could be numerous metabolites from one parent drug and it is also practically impossible to purchase or synthesize standards for all the metabolites. An addition consideration is the economical unsoundness of purchasing the standards for all metabolites. As a result, current targeted analysis of metabolites using tandem mass spectrometry is only limited to pharmaceuticals whose metabolites are well "known" from clinical data and standards for the metabolites are readily available to confirm the identity (Miao and Metcalfe 2003; Gobel et al. 2004; Miao et al. 2005). However, the increasing number of studies reporting the presence of metabolites in the environment underlines the urge to include non-target or unknown metabolites as an important part of routine analysis of pharmaceuticals. For instance, several LC-MS methods have been developed to determine carbamazepine, one of the most frequently detected pharmaceuticals in WWTP effluents and surface water, along with its major phase I metabolites in aqueous samples (Miao and Metcalfe 2003; Miao et al. 2005). Interestingly, metabolite 10,11-dihydro-10,11- 
dihydroxycarbamazepine was found to be the dominant analyte which had a concentration 3 to 4 times higher than that of carbamazepine in wastewater (Miao and Metcalfe 2003). A significant increase of the parent drug was also observed in the treated wastewater effluent relative to concentrations in the untreated wastewater influent, suggesting that some phase II metabolites could have been transformed to the free form by cleavage of the conjugates during the treatment. The increase of the parent drug is likely related to the fact that hydroxylated metabolites of carbamazepine occur primarily in conjugated forms such as glucuronidation in body fluids (Miao et al. 2005). A more recent study provided the first evidence that phase II metabolites of sulfamethoxazole existed and could be back-transformed to its parent drug under abiotic conditions, indicating that these phase II metabolites may survive wastewater treatment and serve as an additional environmental source of sulfamethoxazole (Bonvin et al. 2012). Therefore, it is increasingly important to include phase II metabolites when assessing the occurrence, fate and transport of pharmaceuticals in the environment.

In recent years, high resolution mass spectrometry including time of flight (TOF) and Orbitrap has become a powerful tool at identifying "unknown" metabolites in both metabolomics and environmental studies (Lim et al. 2007; Ferrer and Thurman 2010; Calza et al. 2012; Thurman and Ferrer 2012). Operated at a resolving power $>40,000$, mass accuracy $<5$ ppm is normally achievable on modern TOF instruments allowing positive identification based on accurate mass 
measurements. Difficulties associated with using TOF instruments are the limited dynamic range and the dependence on lock mass or internal calibration to maintain high mass accuracy (Petrovic et al. 2006; Ibanez et al. 2012). In contrast, external calibration can be used for Orbitrap instruments to obtain excellent mass accuracy $(<3 \mathrm{ppm})$ resulting a simplified operational protocol (Lim et al. 2007). In addition, Orbitrap instruments offer better dynamic range and sensitivity close to those of many triple quadrupole instruments. The features that Orbitrap offers are especially important for environmental analysis when the concentrations are not sufficiently high compared to those in metabolic or pharmacokinetic studies (Peterman et al. 2006).

The objectives in this study were to identify phase II metabolites in reclaimed water using a $Q$ Exactive Orbitrap mass spectrometer based on accurate mass measurements combined with characteristic ions from MS/MS data dependent scans, and to determine the concentrations of identified phase II metabolites relative to their parent drugs in reclaimed water.

\subsection{Experimental}

\subsubsection{Chemicals and Standards}

Reference standards of pharmaceuticals, two metabolites (norfluxetine and Odesmethyltramadol) and isotopically labeled standards were purchased from Sigma-Aldrich (Milwaukee, WI), MP Biomedicals, LLC. (Santa Ana, CA), Cerilliant Corp. (Round Rock, TX), American Radiolabeled Chemicals,Inc. (St 
Louis, MO), Toronto Research Chemicals Inc. (North York, Ontario), ACROS organics (Morris Plains, NJ), C/D/N Isotopes Inc. (Pointe-Claire, Quebec) and Cambridge Isotopes Lab. Inc. (Andover, MA). Details of the sources of standards were also shown in Table 4.1. All standards presented purity higher than 95\%. Pierce LTQ Velos ESI positive ion and negative ion calibration solutions (Thermo Scientific, Rockford, IL) for Q Exactive orbitrap mass spectrometer were provided by Thermo Scientific (Rockford, IL). LC-MS grade methanol, water and formic acid were purchased from Fisher Scientific (Bridgewater, NJ). The $0.1 \%$ formic acid used as mobile phase was prepared daily. All stock solutions (100 ppm) and working solutions were made in methanol and stored in the dark at $-20^{\circ} \mathrm{C}$.

\subsubsection{Sample Collection}

During a period of one month, reclaimed water $(500 \mathrm{~mL})$ was directly drawn from the reclaimed water outlet at Florida International University Biscayne Bay Campus (North Miami, FL). Polyethylene terephthalate bottles $(500 \mathrm{~mL})$ were used to collect water samples and rinsed with the sampling water three times before the samples were taken. Water samples were filtered and stored in dark at or below $4{ }^{\circ} \mathrm{C}$ until processed. Head space was minimized by filling the bottles completely. Water extraction was conducted within 7 days of collection to prevent changes associated with biodegradation. 


\subsubsection{Sample Extraction}

To remove suspended materials, water samples were filtered through $0.5 \mu \mathrm{m}$ pre-combusted glass fiber filters (GE Water \& Process Technologies, Trevose, PA) within $24 \mathrm{~h}$ after collection. Each water sample was extracted with an Oasis HLB (3 cc/60 mg, Waters Corp., Franklin, MA) cartridge coupled in tandem to a Sep-Pak tC18 Plus cartridge (900 mg, Waters Corp., Franklin, MA). The combination of two different sorbent materials was used to ensure high recoveries of the metabolites (Eichhorn et al. 2005). Both cartridges were conditioned together with $5 \mathrm{~mL}$ of methanol followed by $5 \mathrm{~mL}$ of distilled water. Solid phase extraction was performed on an ALLTECH 12-port vacuum manifold (Deerfield, IL). After the samples had passed through the cartridges, the analytes were eluted with $3 \mathrm{~mL}$ of methanol. The methanol eluent was evaporated to dryness under a gentle stream of purified nitrogen gas. Dried residues were reconstituted with $200 \mu \mathrm{L}$ of methanol:0.1\% formic acid $50: 50(\mathrm{v} / \mathrm{v})$. After brief sonication and vortex, the samples were ready for LC-MS analysis.

\subsubsection{Liquid Chromatography and Q Exactive Mass spectrometry}

Liquid chromatography was performed on a quaternary Thermo Scientific Accela pump with a Thermo PAL CTC autosampler. A Hypersil GOLD $50 \times 2.1 \mathrm{~mm}, 3$ $\mu \mathrm{m}$ particle size and $175 \AA$ pore size column from Thermo Scientific (Bellefonte, PA) was used for separation. Two sets of mobile phases were used for both positive and negative ionization modes. The mobile phases used for positive 
ionization mode consisted of $A, 0.1 \%$ formic acid in water $(v / v)$ and $B, 100 \%$ acetonitrile. Acetonitrile and water were used for negative ionization mode. The gradient remained the same for both ionization modes, which was (acetonitrile $\%$ ): $0 \min 3 \%, 2 \min 3 \%, 8.5 \min 97 \%, 15 \min 3 \%$ and $17 \min 3 \%$. A flow rate of $300 \mu \mathrm{L} / \mathrm{min}$ was maintained for both gradients. The injection volume was set to $10 \mu \mathrm{L}$.

All extracts were analyzed by a $Q$ Exactive Orbitrap mass spectrometer (Thermo Scientific, San Jose, CA) equipped with a heated electrospray ion source (HESIII) operated in both positive and negative modes. As a part of routine maintenance, the instrument was calibrated weekly using Pierce LTQ Velos ESI Positive Ion and Negative Ion Calibration Solutions (Thermo Scientific, Rockford, IL) for positive and negative modes, respectively. Mass accuracy less than 0.6 ppm was always achieved from the external calibration that was performed prior to analysis or every 24 hours. Ultra-pure nitrogen and argon were used as ion source gas and collision gas, respectively. Mass spectrometry parameters in positive mode were set as follows: Sheath gas, Aux gas and Sweep gas were at 35,35 and 5 arbitrary units, respectively. Similarly, the parameters in negative mode were set as follows: Sheath gas, Aux gas and Sweep gas were at 40, 20 and 5 arbitrary units, respectively. The following parameters remained the same for both positive and negative modes: Spray voltage was at $4 \mathrm{kV}$. Capillary temperature and Vaporizer temperature were at $300{ }^{\circ} \mathrm{C}$. The data-dependent scan cycle included a full scan operated at a resolving power of 70,000 with a 
scan range from $80-1000 \mathrm{~m} / \mathrm{z}$ and three corresponding data-dependent MS/MS scans acquired at resolving power of 35,000 . The three most abundant precursor ions triggered data-dependent scanning and were subsequently injected to the C-trap to be fragmented. Isolation window for MS/MS scanning was set to $2 \mathrm{~m} / \mathrm{z}$. Normalized collision energy (NCE) was set to 35 with stepped NCE of $25 \%$.

\subsubsection{Data Interpretation}

The calculation of exact masses from elemental compositions was carried out using MetWorks 1.3 (Thermo Scientific, San Jose, CA). High resolution extracted ion chromatograms (XICs) of the parent pharmaceutical and its potential phase II metabolites were obtained by processing the full scan data using MetWorks with a $5 \mathrm{ppm}$ mass tolerance. Data-dependent spectra of the positively identified compounds were further investigated by Mass Frontier 7.0 (Thermo Scientific, San Jose, CA) using the Fragment lon Search (FISh) feature, in which theoretical spectra generated from chemical structures were compared with those observed from the experiment. Similarities between the theoretical and observed spectra, along with isotope patterns, even electron species and rings plus double bonds (RDB) values were considered to aid the assignment of metabolite identification. 


\subsection{Results and discussions}

\subsubsection{Performance of Q Exactive Orbitrap}

Table 5.1 shows the selected pharmaceuticals routinely monitored in reclaimed water as described in previous studies. The performance of accurate mass measurements in external calibration mode was investigated by injecting $10 \mu \mathrm{L}$ of the mixture of all parent pharmaceutical standards at $200 \mathrm{ng} / \mathrm{mL}$ immediately after calibration and at the end of each sequence up to $16 \mathrm{~h}$ post-calibration. The observed accurate masses were systematically lower than exact masses for the majority of compounds, but the mass accuracy never exceeded $2.9 \mathrm{ppm}$ except for naproxen which was calculated at $-3.9 \mathrm{ppm}$ (Table 5.1). Mass accuracy was also evaluated for the standards injected at the end of each sequence. Mass accuracy of $4 \mathrm{ppm}$ or better was always achieved for all compounds after up to $16 \mathrm{~h}$ post-calibration (data not shown). The values for mass accuracy were better than those obtained from most time-of-flight mass spectrometers in internal calibration mode (Petrovic et al. 2006; Ferrer and Thurman 2010; Thurman and Ferrer 2012).

Table 5.1 Elemental compositions, exact masses, observed accurate masses, and mass accuracy of selected parent pharmaceuticals

\begin{tabular}{clllc}
\hline \multirow{2}{*}{ Name } & Elemental & Exact & Observed & Mass \\
& Composition & Mass $(\mathrm{m} / \mathrm{z})$ & $\begin{array}{c}\text { Accurate } \\
\text { Mass }(\mathrm{m} / \mathrm{z})\end{array}$ & $\begin{array}{c}\text { Accuracy } \\
(\mathrm{ppm})\end{array}$ \\
Atenolol & $\mathrm{C} 14 \mathrm{H} 22 \mathrm{~N} 2 \mathrm{O} 3$ & 267.1703 & 267.1696 & -2.6
\end{tabular}




\begin{tabular}{|c|c|c|c|c|}
\hline Atorvastatin & C33H35FN2O5 & 559.2603 & 559.2593 & -1.8 \\
\hline Betaxolol & $\mathrm{C} 18 \mathrm{H} 29 \mathrm{NO} 3$ & 308.2220 & 308.2213 & -2.3 \\
\hline Bezafibrate $^{a}$ & $\mathrm{C} 19 \mathrm{H} 20 \mathrm{CINO} 4$ & 360.1008 & 360.1011 & 0.8 \\
\hline Butalbital $^{a}$ & $\mathrm{C} 11 \mathrm{H} 16 \mathrm{~N} 2 \mathrm{O} 3$ & 223.1088 & 223.1085 & -1.3 \\
\hline Caffeine & $\mathrm{C} 8 \mathrm{H} 10 \mathrm{~N} 4 \mathrm{O} 2$ & 195.0877 & 195.0873 & -2.1 \\
\hline Carazolol & $\mathrm{C} 18 \mathrm{H} 22 \mathrm{~N} 2 \mathrm{O} 2$ & 299.1754 & 299.1747 & -2.3 \\
\hline Carbamazepine & $\mathrm{C} 15 \mathrm{H} 12 \mathrm{~N} 2 \mathrm{O}$ & 237.1022 & 237.1017 & -2.1 \\
\hline Clenbuterol & $\mathrm{C} 12 \mathrm{H} 18 \mathrm{Cl} 2 \mathrm{~N} 2 \mathrm{O}$ & 277.0869 & 277.0863 & -2.2 \\
\hline Clotrimazole & $\mathrm{C} 22 \mathrm{H} 17 \mathrm{CIN} 2$ & 345.1153 & 345.1146 & -2.0 \\
\hline Codeine & $\mathrm{C} 18 \mathrm{H} 21 \mathrm{NO} 3$ & 300.1594 & 300.1588 & -2.0 \\
\hline Diazepam & $\mathrm{C} 16 \mathrm{H} 13 \mathrm{CIN} 2 \mathrm{O}$ & 285.0789 & 285.0785 & -1.4 \\
\hline Diclofenac $^{a}$ & $\mathrm{C} 14 \mathrm{H} 11 \mathrm{Cl} 2 \mathrm{NO} 2$ & 294.0094 & 294.0097 & 1.0 \\
\hline Diltiazem & $\mathrm{C} 22 \mathrm{H} 26 \mathrm{~N} 2 \mathrm{O} 4 \mathrm{~S}$ & 415.1686 & 415.1677 & -2.2 \\
\hline Diphenhydramine & $\mathrm{C} 17 \mathrm{H} 21 \mathrm{NO}$ & 256.1696 & 256.1690 & -2.3 \\
\hline Enalapril & $\mathrm{C} 2 \mathrm{OH} 28 \mathrm{~N} 2 \mathrm{O} 5$ & 377.2071 & 377.2062 & -2.4 \\
\hline Enalaprilat & $\mathrm{C} 18 \mathrm{H} 24 \mathrm{~N} 2 \mathrm{O} 5$ & 349.1758 & 349.1751 & -2.0 \\
\hline Erythromycin & C37H65NO12 & 716.4580 & 716.4562 & -2.5 \\
\hline Fenofibrate & $\mathrm{C} 2 \mathrm{OH} 21 \mathrm{ClO} 4$ & 361.1201 & 361.1191 & -2.8 \\
\hline Fluoxetine & C17H18F3NO & 310.1413 & 310.1409 & -1.3 \\
\hline Furosemide $^{a}$ & $\mathrm{C} 12 \mathrm{H} 11 \mathrm{CIN} 2 \mathrm{O} 5 \mathrm{~S}$ & 329.0004 & 329.0009 & 1.5 \\
\hline Gemfibrozil $^{a}$ & $\mathrm{C} 15 \mathrm{H} 22 \mathrm{O} 3$ & 249.1496 & 249.1495 & -0.4 \\
\hline Glibenclamide & C23H28CIN3O5S & 494.1511 & 494.1503 & -1.6 \\
\hline Ibuprofen $^{a}$ & $\mathrm{C} 13 \mathrm{H} 18 \mathrm{O} 2$ & 205.1234 & 205.1228 & $-2 . \subseteq$ \\
\hline Ketoprofen $^{a}$ & $\mathrm{C} 16 \mathrm{H} 14 \mathrm{O} 3$ & 253.0870 & 253.0873 & 1.2 \\
\hline Lincomycin & C18H34N2O6S & 407.2210 & 407.2202 & -2.0 \\
\hline Lorazepam & $\mathrm{C} 15 \mathrm{H} 10 \mathrm{Cl} 2 \mathrm{~N} 2 \mathrm{O} 2$ & 321.0192 & 321.0186 & -1.9 \\
\hline Mefenamic acid $^{a}$ & $\mathrm{C} 15 \mathrm{H} 15 \mathrm{NO} 2$ & 240.1030 & 240.1030 & 0.0 \\
\hline Metoprolol & $\mathrm{C} 15 \mathrm{H} 25 \mathrm{NO} 3$ & 268.1907 & 268.1902 & -1.9 \\
\hline Metronidazole & C6H9N3O3 & 172.0717 & 172.0713 & -2.3 \\
\hline Mevastatin & $\mathrm{C} 23 \mathrm{H} 34 \mathrm{O} 5$ & 391.2479 & 391.2471 & -2.0 \\
\hline Nadolol & $\mathrm{C} 17 \mathrm{H} 27 \mathrm{NO} 4$ & 310.2013 & 310.2005 & -2.6 \\
\hline Naproxen ${ }^{a}$ & $\mathrm{C} 14 \mathrm{H} 14 \mathrm{O} 3$ & 229.0870 & 229.0861 & -3.9 \\
\hline Norfluoxetine & $\mathrm{C} 16 \mathrm{H} 16 \mathrm{~F} 3 \mathrm{NO}$ & 296.1257 & 296.1250 & \\
\hline
\end{tabular}




$\begin{array}{llllr}\text { Paroxetine } & \text { C19H20FNO3 } & 330.1500 & 330.1491 & -2.7 \\ \text { Pentobarbital }^{a} & \mathrm{C} 11 \mathrm{H} 18 \mathrm{~N} 2 \mathrm{O} 3 & 225.1245 & 225.1240 & -2.2 \\ \text { Phenobarbital }^{a} & \mathrm{C} 12 \mathrm{H} 12 \mathrm{~N} 2 \mathrm{O} 3 & 231.0775 & 231.0771 & -1.7 \\ \text { Pindolol } & \mathrm{C} 14 \mathrm{H} 20 \mathrm{~N} 2 \mathrm{O} 2 & 249.1598 & 249.1593 & -2.0 \\ \text { Pravastatin } & \mathrm{C} 23 \mathrm{H} 36 \mathrm{O} 7 & 447.2353 & 447.2348 & -1.1 \\ \text { Propranolol } & \mathrm{C} 16 \mathrm{H} 21 \mathrm{NO} 2 & 260.1645 & 260.1639 & -2.3 \\ \text { Salbutamol } & \mathrm{C} 13 \mathrm{H} 21 \mathrm{NO} 3 & 240.1594 & 240.1591 & -1.2 \\ \text { Sertraline } & \mathrm{C} 17 \mathrm{H} 17 \mathrm{Cl} 2 \mathrm{~N} & 306.0811 & 306.0807 & -1.3 \\ \text { Sotalol } & \mathrm{C} 12 \mathrm{H} 20 \mathrm{~N} 2 \mathrm{O} 3 \mathrm{~S} & 273.1267 & 273.1261 & -2.2 \\ \text { Sulfamethoxazole } & \mathrm{C} 10 \mathrm{H} 11 \mathrm{~N} 3 \mathrm{O} 3 \mathrm{~S} & 254.0594 & 254.0589 & -2.0 \\ \text { Tamoxifen } & \mathrm{C} 26 \mathrm{H} 29 \mathrm{NO} & 372.2322 & 372.2315 & -1.9 \\ \text { Timolol } & \mathrm{C} 13 \mathrm{H} 24 \mathrm{~N} 4035 & 317.1642 & 317.1640 & -0.6 \\ \text { Trimethoprim } & \mathrm{C} 14 \mathrm{H} 18 \mathrm{~N} 403 & 291.1452 & 291.1445 & -2.4 \\ \text { Compounds detected in negative mode }[\mathrm{M}-\mathrm{H}]^{-} & & \end{array}$

\subsubsection{Tentative Identification of Phase II metabolites based on Accurate Mass}

Because this study focuses on phase II metabolites, the identification procedure will only be discussed for the cases when both parent drug and at least one of its metabolites are present together. On the basis of the fact that most phase II metabolites are more water soluble than the corresponding parent drugs (Ferrer and Thurman 2010), with the exception of acetylation (Gobel et al. 2004), an earlier retention time of a metabolite is a positive indication when tentatively identifying metabolites based on accurate mass. Moreover, it is difficult to be certain if the observed peak is a metabolite or another isomer without knowing the chromatographic behavior of the parent drug (Ferrer and Thurman 2012). 
Peaks from full scan chromatograms that show intensities lower than $1 \times 10^{5}$ counts will not be considered because the intensity would not be high enough to trigger a data-dependent MS/MS scan, which is essential for structure elucidation.

Upon inputting the elemental composition and polarity of each selected pharmaceutical into MetWorks, XICs for the parent drug along with its most commonly found phase II metabolites are created based on the exact masses of modifications using a mass tolerance at $5 \mathrm{ppm}$. These calculations were shown in Table 5.2.

Table 5.2 Monitored phase II modifications and exact masses of the modifications to parent pharmaceuticals

Phase II Modification

Glucuronidation

Glutathione conjugation

Sulfation

Acetylation

Glycine conjugaion

Cysteine conjuation

Taurine conjugation

S-cysteine conjugation

$\mathrm{N}$-acetylcysteine conjugation

Hydroxylation and Glucuronidation

Decarboxylation and Glucuronidation
Exact Mass of

Modification

$+176.0321$

$+307.0838$

$+79.9568$

$+42.0106$

$+57.0215$

$+103.0092$

$+107.0041$

$+119.0041$

$+161.0147$

$+192.0270$

$+148.0372$ 
Hydroxylation and Sulfation

De-ethylation + Glucuronidation

De-methylation + Glucuronidation

Epoxidation + S-GSH conjugation

Desaturation + S-GSH conjugation
$+95.9517$

$+148.0008$

$+162.0164$

$+323.0787$

$+305.0682$

A detailed examination of the XICs for all potential metabolites in reclaimed water samples revealed that sulfamethoxazole glucuronide and acetylsulfamethoxazole, two phase II metabolites of sulfamethoxazole, appeared at retention times of $5.38 \mathrm{~min}$ and $5.56 \mathrm{~min}$, respectively (Figure 5.1). Meanwhile, a peak at 5.46 min was also observed for sulfamethoxazole as shown in Figure 5.1. The retentive behaviors of these compounds were in good agreement with those reported in literature (Gobel et al. 2004), in which sulfamethoxazole eluted later than sulfamethoxazole glucuronide but earlier than acetyl-sulfamethoxazole. The accurate mass of sulfamethoxazole was observed at $\mathrm{m} / \mathrm{z} 254.0590$, which is $-1.6 \mathrm{ppm}$ from its exact mass at $\mathrm{m} / \mathrm{z} 254.0594$. For verification purposes, the mixture containing all parent pharmaceutical standards at $200 \mathrm{ng} / \mathrm{mL}$ was injected onto the column, and sulfamethoxazole standard was eluted at the same retention time with the same measured accurate mass (m/z 254.0590). Therefore, sulfamethoxazole can be identified in reclaimed water samples based on retention time and its elemental composition (less than $5 \mathrm{ppm}$ ). Additional confirmation was done by comparing MS/MS spectra with those obtained from sulfamethoxazole standard (Figure 5.2). 


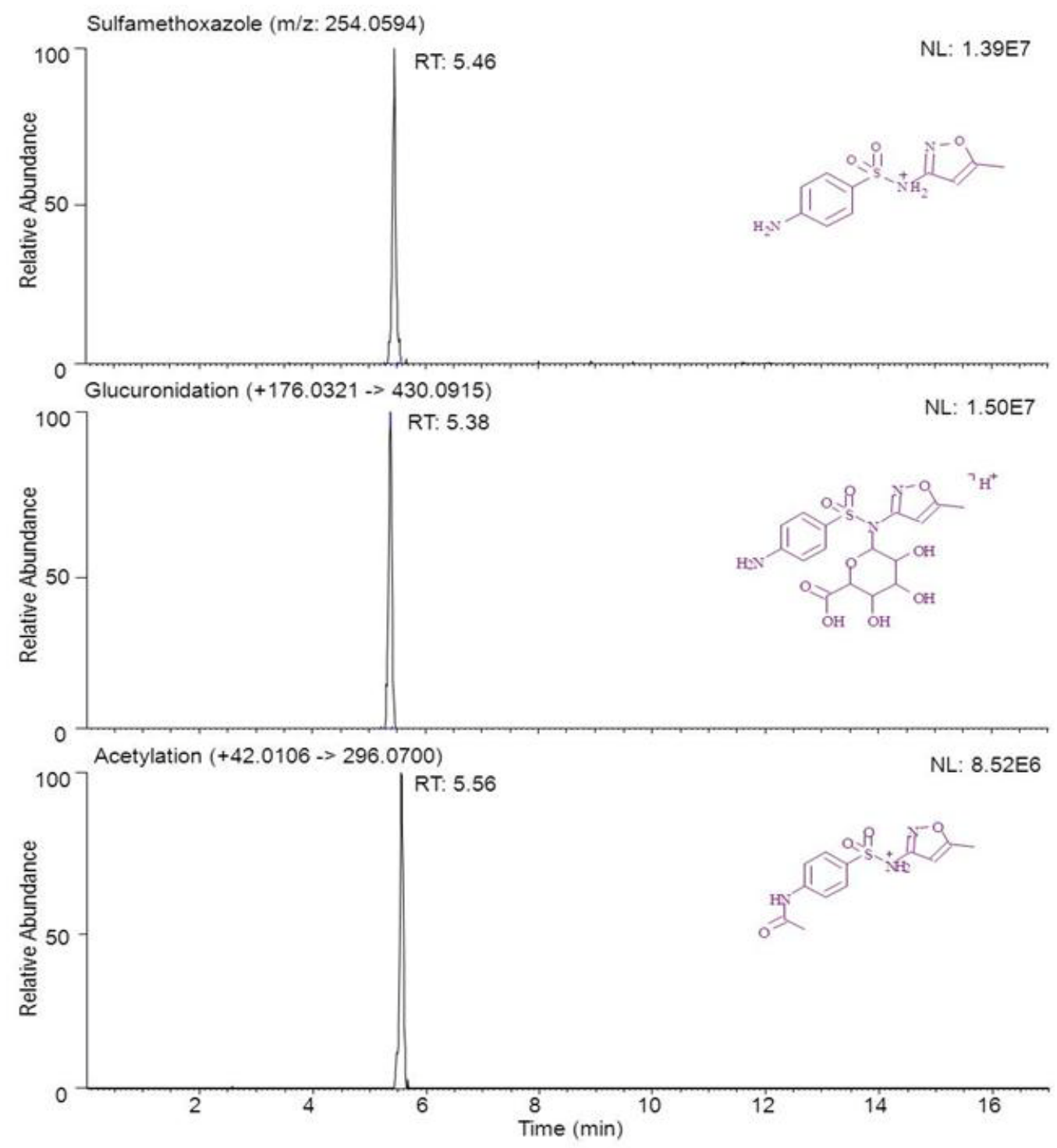

Figure 5.1 LC-Q Exactive Orbitrap extracted ion chromatograms (XICs) of sulfamethoxazole ( $\mathrm{m} / \mathrm{z} 254.0594)$, sulfamethoxazole glucuronide ( $\mathrm{m} / \mathrm{z} 430.0915)$ and acetyl-sulfamethoxazole $(\mathrm{m} / \mathrm{z} 296.0700)$ in a reclaimed water sample; mass tolerance was set to $5 \mathrm{ppm}$ for all compounds

The accurate masses for both metabolites were measured at $\mathrm{m} / \mathrm{z} 430.0916$ and $\mathrm{m} / \mathrm{z} 296.0695$ (mass accuracy less than $1.7 \mathrm{ppm}$ from their exact masses), 
respectively, which correspond to the addition of glucuronic acid (176 Da) and acetyl group (42 Da) to sulfamethoxazole. These metabolites can only be tentatively identified at this point because standards and their detailed fragmentation patterns are needed to ensure their identities. Unfortunately, unlike metabolic studies where there are metabolite-free blank samples to compare with, and more importantly, there are much fewer compounds to deal with, environmental studies monitor dozens, sometimes hundreds of compounds which make purchasing or synthesizing metabolite standards for all compounds unrealistic and economically unsound. An alternative that could be applied here when metabolite standards are not readily available is to compare the similarities of fragmentation patterns between parent drug and its phase II metabolites as they share the "back bone" in their molecular structures (Lim et al. 2007; Thurman and Ferrer 2012). Moreover, combining high resolution accurate mass measurements and tandem mass spectrometry increases the confidence of the proposed structures of product ions. The availability of chemical formulas for all precursor and product ions, irrespective of relative ion abundance, generated from high resolution data-dependent spectra can also accelerate the structure elucidation of metabolites (Ferrer and Thurman 2010; Thurman and Ferrer 2012). Therefore, data-dependent spectra showing fragmentation patterns of sulfamethoxazole glucuronide and acetyl-sulfamethoxaozle were further investigated against that obtained for sulfamethoxazole using Mass Frontier. 


\subsubsection{Identification of Acetyl-sulfamethoxazole and Sulfamethoxazole Glucuronide}

The high resolution data-dependent MS/MS spectrum of $\mathrm{m} / \mathrm{z} 254.0590$ at retention time $5.46 \mathrm{~min}$ in the reclaimed water extract was shown In Figure 5.2B, which was nearly identical with that observed for sulfamethoxazole standard (Figure 5.2A). The two most abundant fragment ions at $156 \mathrm{Da}$ and $108 \mathrm{Da}$ were in good agreement with those reported in previous studies (Sacher et al. 2001; Vanderford et al. 2003). The structures of all major fragments were predicted by Mass Frontier on the basis of the fragmentation rules built into the software and labeled in Figure 5.2A and 5.2B. All the labeled fragment ions in Figure 5.2B matched within $1.8 \mathrm{ppm}$ and satisfied the ion fragments predicted by Mass Frontier. The proposed fragmentation pathway is also shown in Figure 5.3A. Therefore, these high resolution MS/MS data clearly showed that the ion $\mathrm{m} / \mathrm{z}$ 254.0590 was sulfamethoxazole and once again confirmed the presence of sulfamethoxazole in the reclaimed water sample.

Figure 5.2C shows the MS/MS spectrum of $\mathrm{m} / \mathrm{z} 296.0695$ at retention time 5.56 min, which corresponds within $-1.7 \mathrm{ppm}$ to the exact mass of acetylsulfamethoxazole. The four most intense fragment ions at $65 \mathrm{Da}, 108 \mathrm{Da}, 134 \mathrm{Da}$ and $198 \mathrm{Da}$ were consistent with those observed by Gobel et al (Gobel et al. 2004) using unit resolution triple quadruple mass spectrometer. All labeled fragment ions in Figure $5.2 \mathrm{C}$ matched within $4.9 \mathrm{ppm}$ and satisfied the ion fragments predicted by Mass Frontier. The proposed fragmentation pathway of 
acetyl-sulfamethoxazole was also described in Figure 5.3B for the first time. Fragment ions at $134 \mathrm{Da}, 150 \mathrm{Da}, 198 \mathrm{Da}$ and $236 \mathrm{Da}$ containing acetyl groups clearly showed that these ions were generated from the acetylation of sulfamethoxazole suggesting that the $\mathrm{m} / \mathrm{z} 296.0695$ was indeed acetylsulfamethoxazole.

Similarly, Figure 5.2D shows the MS/MS spectrum of $\mathrm{m} / \mathrm{z} 430.0916$ at retention time $5.38 \mathrm{~min}$, which corresponds within $0.2 \mathrm{ppm}$ to the exact mass of sulmathoxoazole glucuronide. The $\mathrm{m} / \mathrm{z} 430.0916$ ion lost 176.0330 to form $\mathrm{m} / \mathrm{z}$ 254.0586, which is a loss within $4.5 \mathrm{ppm}$ for the glucuronic acid. The ion $\mathrm{m} / \mathrm{z}$ 254.0586, which is $-3.1 \mathrm{ppm}$ from the exact mass of sulfamethoxazole, further fragmented to give two important ions at $\mathrm{m} / \mathrm{z} 108.0445$ (1.2 ppm mass accuracy) and $\mathrm{m} / \mathrm{z} 156.0110$ (-2.8 ppm mass accuracy), which were also observed as the two most abundant fragment ions for sulfamethxoazole standard (Figure 5.2A). These two ions together indicated that $\mathrm{m} / \mathrm{z} 254.0586$ was indeed sulfamethoxazole formed on the basis of the de-conjugation of glucuronic acid. The fragmentation pathway of the glucuronide conjugate was shown in Figure 5.3C. Thus, the evidence is strong that the ion $\mathrm{m} / \mathrm{z} 430.0916$ is sulfamethoxazole glucuronide. 

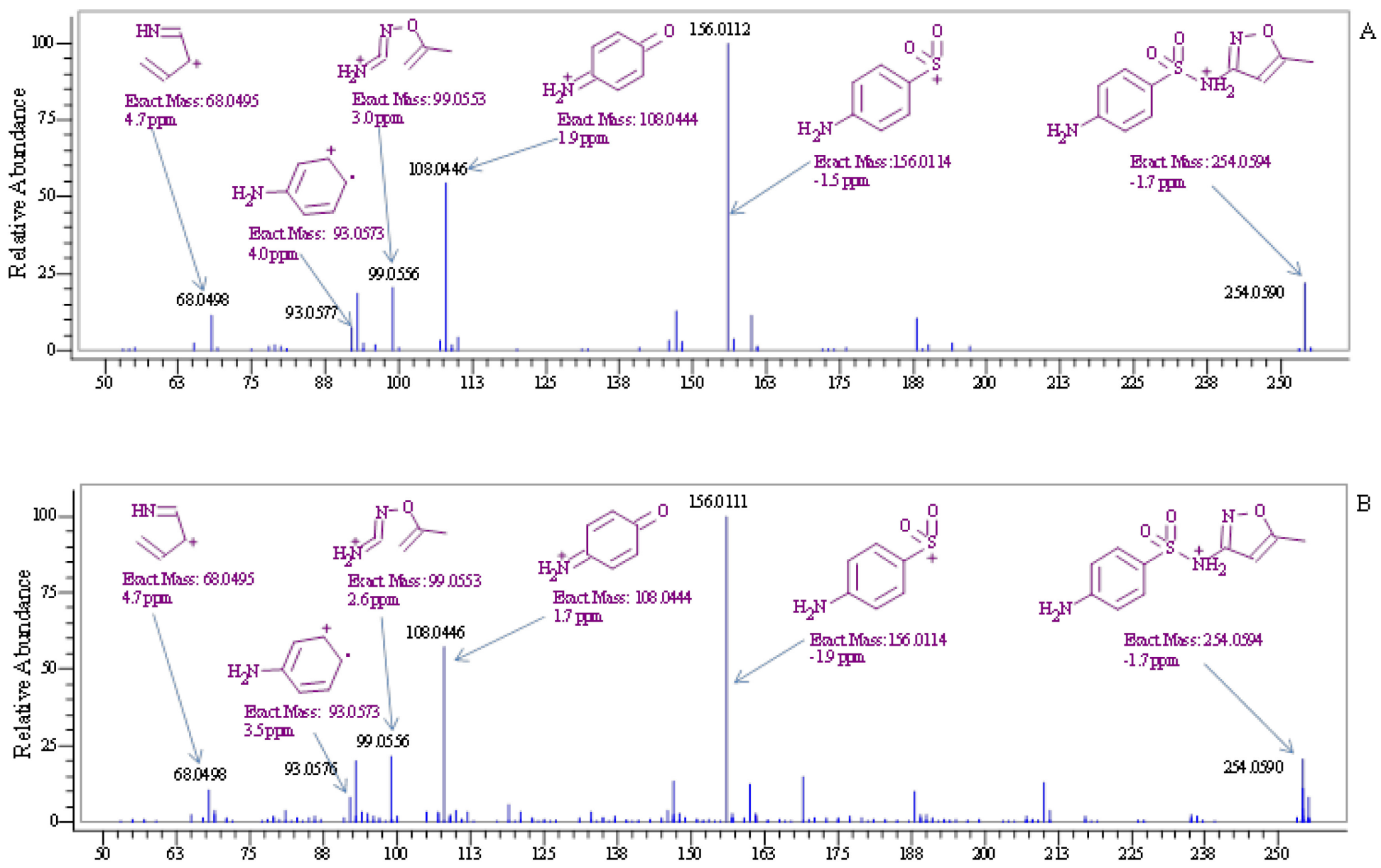

99 


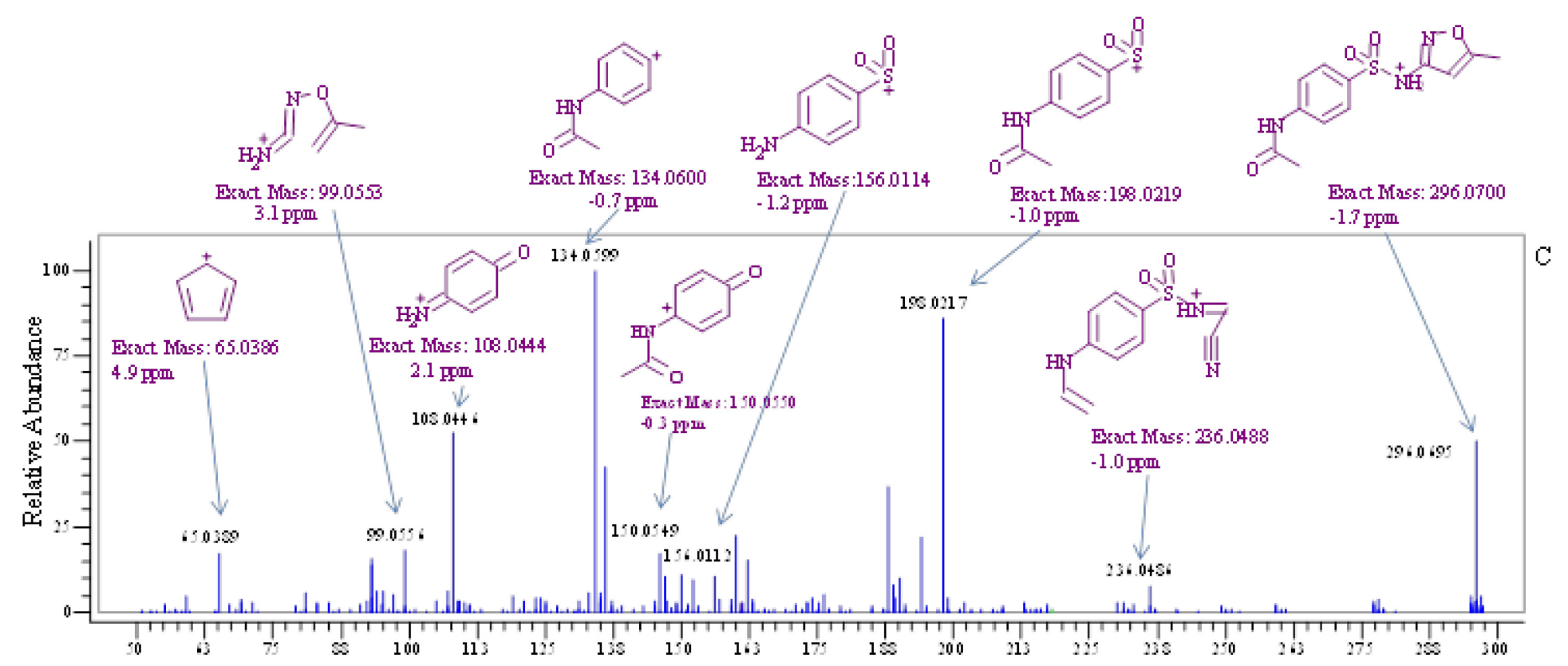




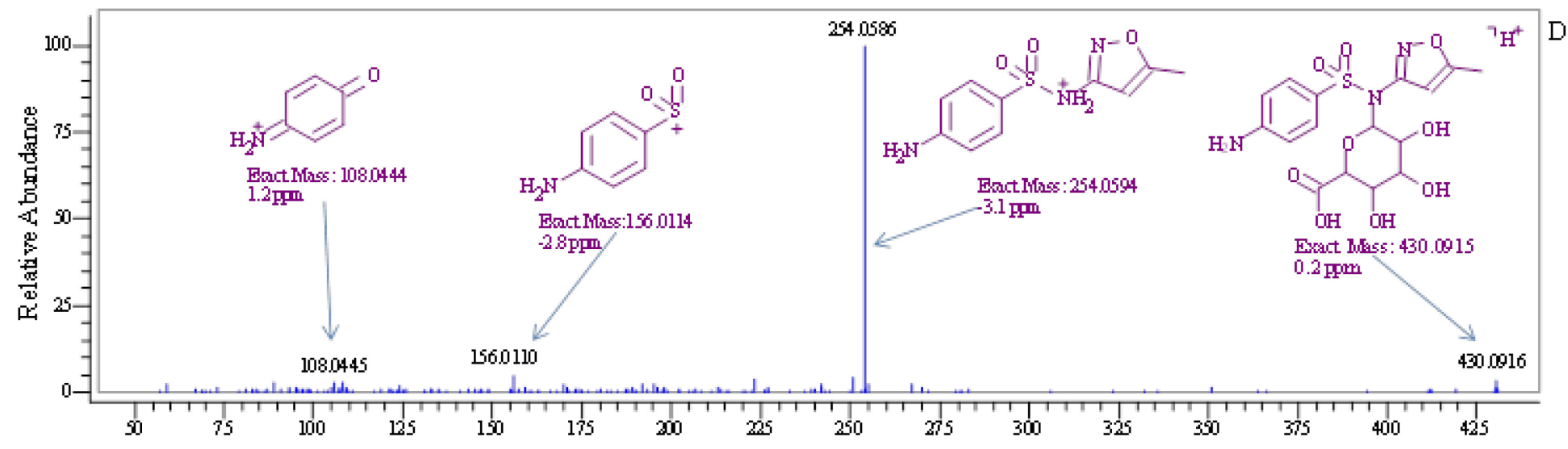

Figure 5.2 High resolution data-dependent MS/MS spectra and major fragments for A: sulfamethoxazole standard, B:

sulfamethoxazole in reclaimed water, C: acetyl-sulfamethoxazole in reclaimed water, and D: sulfamethoxazole glucuronide in reclaimed water 
A
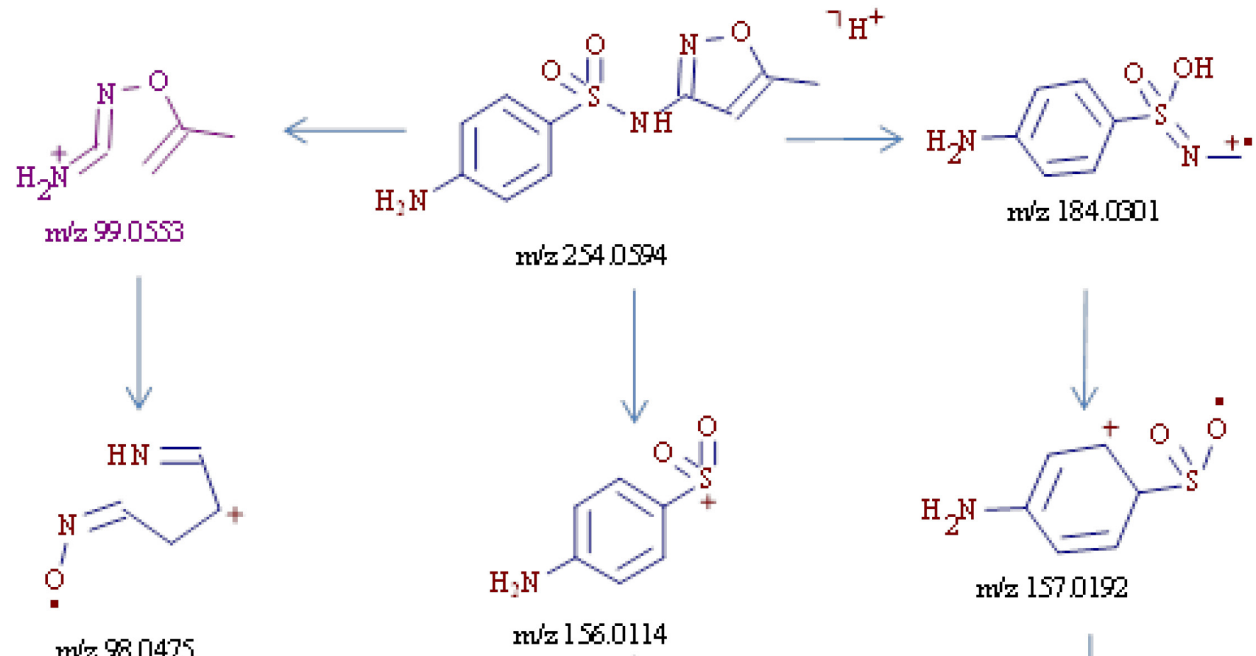

miz 90475
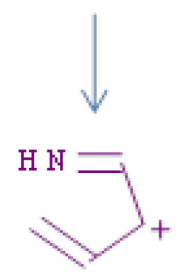

riv 600495

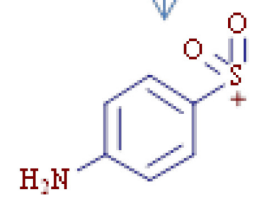

miz 1560114
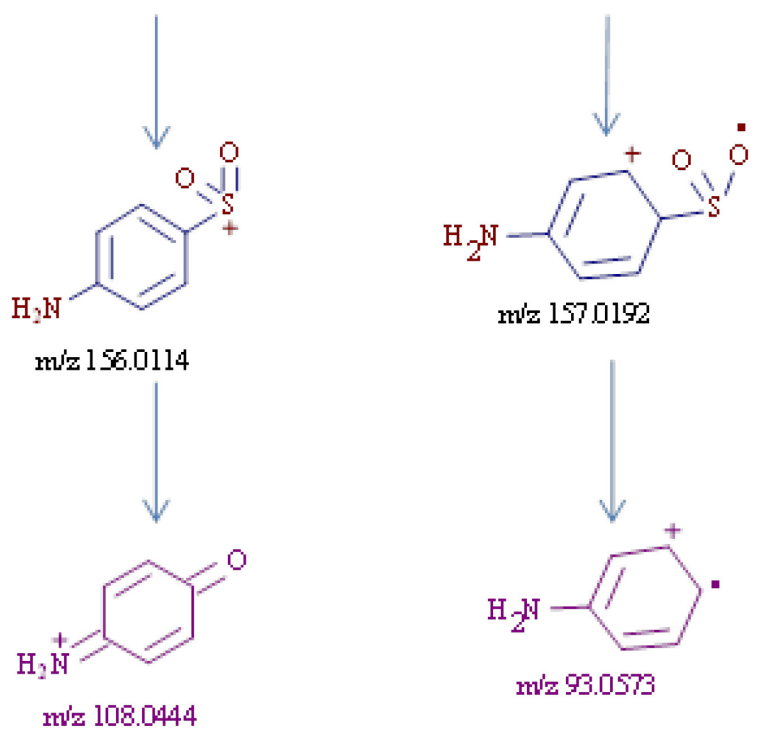
$\mathrm{B}$

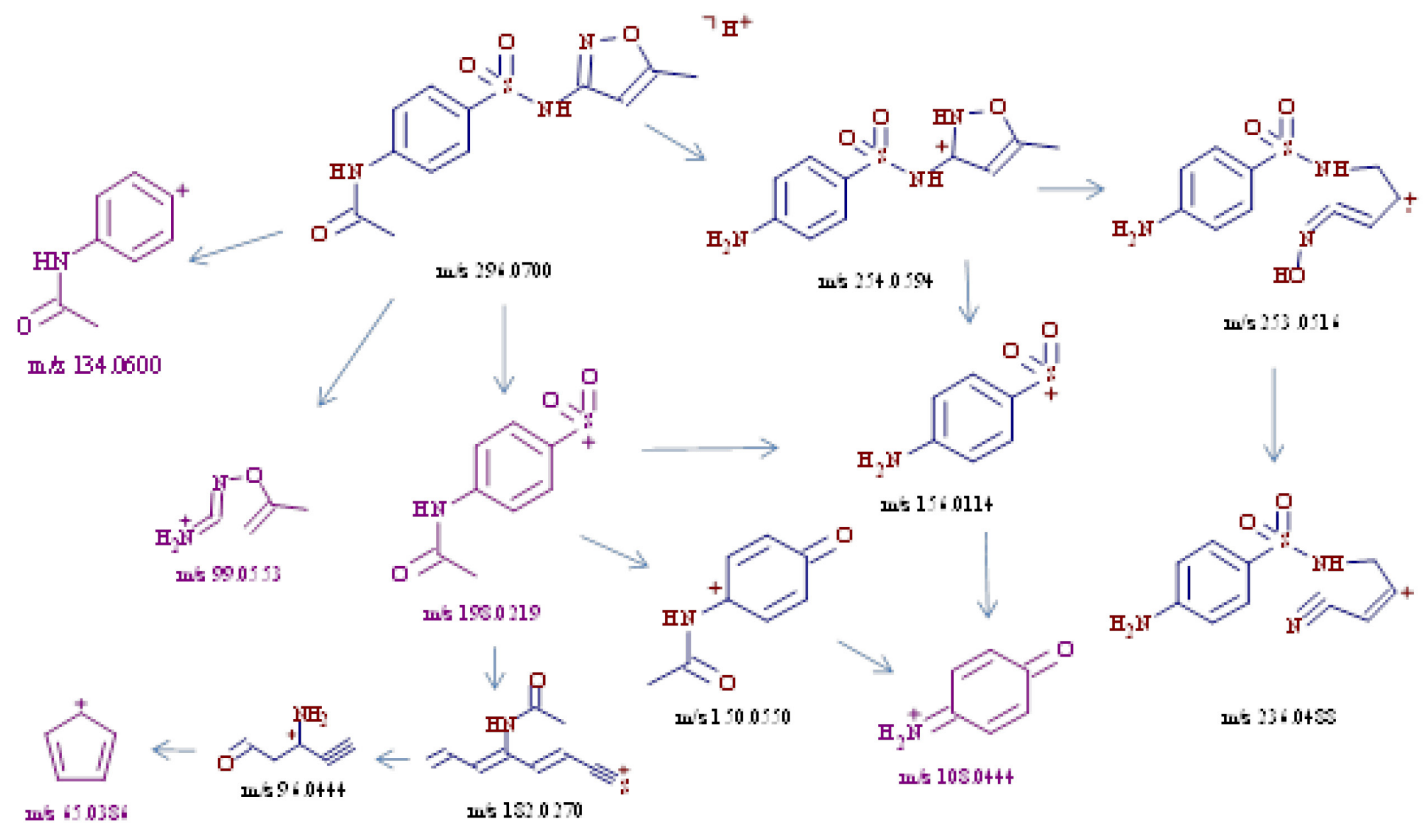

C

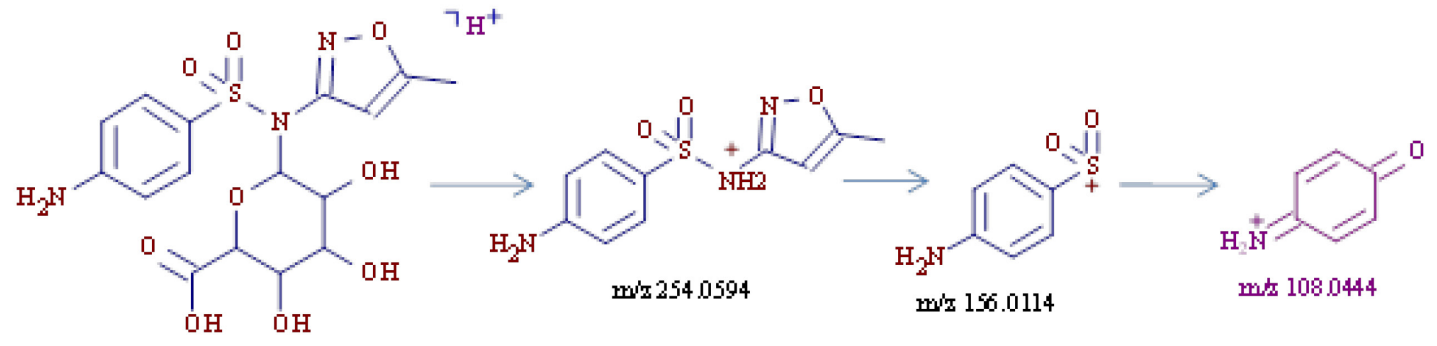

$\min 430.0915$

Figure 5.3 Proposed fragmentation pathways for A: sulfamethoxazole, B: acetylsulfamethoxazole, and C: sulfamethoxazole glucuronide

\subsubsection{Determination of Sulfamethoxazole and Its Metabolites in Reclaimed}

\section{Water}

Collected reclaimed water samples were spiked with sulfamethoxazole-d4 (exact mass at $\mathrm{m} / \mathrm{z} 258.0845$ ) as surrogate internal standard and extracted as 
described above. Quantitation was carried out in MS full scan mode at resolution 70,000 only using the peak area from the extracted ion chromatograms of the base peak ion shown in table 5.1 , using a mass tolerance of 5 ppm. Calibration curve for sulfamethoxazole was built at concentrations ranging from $0.1 \mathrm{ng} / \mathrm{mL}$ to $200 \mathrm{ng} / \mathrm{mL}$, with $R^{2}>0.99$. Method detection limit for sulfamethoxazole in reclaimed water was determined at $67 \mathrm{ng} / \mathrm{L} \quad(n=7)$. Recovery for sulfamethoxazole was $94 \pm 14 \%(n=3)$. Considering the structural similarity between sulfamethoxazole and its metabolites and the availability of metabolite standards, concentrations for acetyl-sulfamethoxazole and sulfamethoxazole

glucuronide were tentatively determined based on the response factor obtained for sulfamethoxazole, regardless of potential matrix effects and ionization efficiency. I was able to achieve this approximation because these compounds were eluted fairly close to each other (within $0.22 \mathrm{~min}$ ), thus may have suffered similar suppression or enhancement. Moreover, the aim of this study was to identify them and to determine the abundance of metabolites relative to their parent drugs in environmental water samples. Therefore, retention times and ions found in previous sections are now used for the determination of sulfamethoxazole and its metabolites in reclaimed water samples.

\subsubsection{Occurrence of Sulfamethoxazole and Its Metabolites in Reclaimed Water}

After the two phase II metabolites along with sulfamethoxazole have been successfully identified in one of the reclaimed water extracts of routine monitoring 
for pharmaceuticals, the developed strategy was applied to screen phase II metabolites in the reclaimed water samples collected during a period of one month at Florida International University Biscayne Bay Campus (North Miami, FL). Sulfamethoxazole and its two metabolites are the only group of compounds that have been consistently detected in all the reclaimed water samples suggesting that the waste water treatment plant is fairly effective at removing most of the phase II metabolites. Sulfamethoxazole, on the other hand, may represent one of the few types of pharmaceuticals whose phase II metabolites can survive intact through sewage treatment plants. As shown in Figure 5.4, average concentrations of sulfamethoxazole, acetyl-sulfamethoxazole and sulfamethoxazole glucuronide were calculated at $2848 \pm 1367 \mathrm{ng} / \mathrm{L}, 1980 \pm 1410$ ng/L, and $2859 \pm 1526 \mathrm{ng} / \mathrm{L}$, respectively. The two metabolites represented 54 $\%$, on the basis of mole fraction, of the source of sulfamethoxazole in reclaimed water. With the knowledge that sulfamethoxazole and acetyl-sulfamethoxazole have been previously detected in wastewater, this is the first known report of sulfamethoxazole glucuronide occurring in environmental water samples. It is commonly believed that glucuronide conjugates are unstable during waste water treatment as a result of being used as an energy source for microbes, the presence of sulfamethoxazole glucuronide indicated that there might be certain factors, such as temperature and the complex consortium of bacteria present, that played a role in keeping the conjugates in reclaimed water. Although this is a small sample size and may not be representative for other treatment facilities, it is still important to be aware of that metabolites do pose a possible threat to 
surface water and drinking water sources, and ultimately to confirm the importance of measuring pharmaceutical metabolites in the environment. Therefore, future studies will include more commonly prescribed pharmaceuticals and pay special attention to those undergo extensive phase II metabolism.

\subsection{Conclusions}

The combination of the high resolution Orbitrap mass spectrometry and metabolic profiling software was successfully applied for the analysis of pharmaceutical metabolites in reclaimed water. Sulfamethoxazole and its two phase II metabolites, acetyl-sulfamethoxazole and sulfamethoxazole glucuronide, were detected and successfully identified in reclaimed water samples using this technique. I also illustrated that it was helpful to use characteristic ions and MS/MS spectra to identify a compound when the standard is not available. Preliminary results showed that the method could be easily adapted to the analysis in various matrices such as sewage extracts, surface water and drinking

water. The resulting data can be used to evaluate the performance of reclaimed water treatment procedures, also the fate of pharmaceuticals in wastewater treatment plants. In the case of sulfamethoxazole, the amount of acetylsulfamethoxazole and sulfamethoxazole glucuronide needs to be considered in order to correctly assess the fate of sulfamethoxazole as they represent the major source of sulfamethoxazole in reclaimed water. 


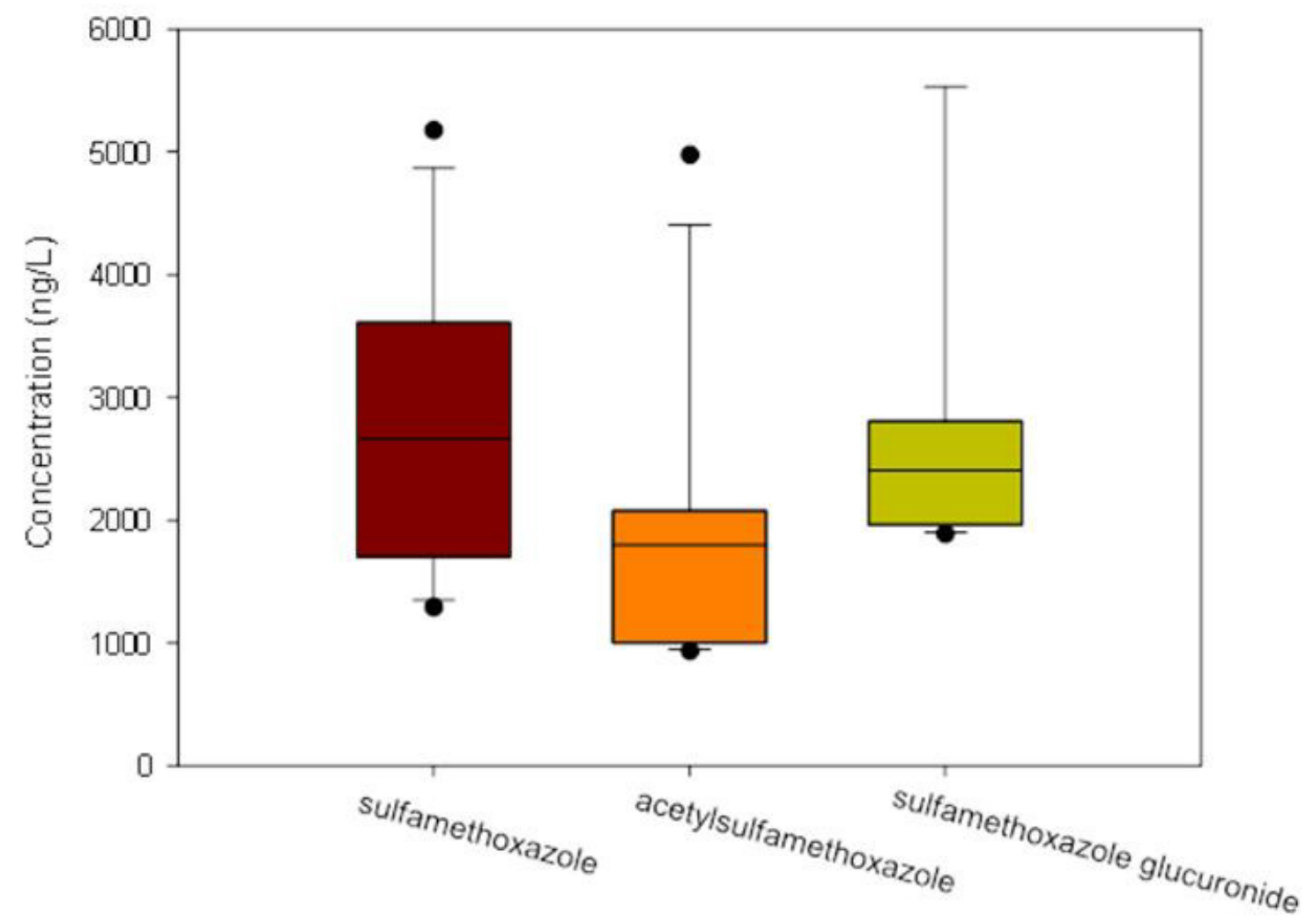

Figure 5.4 Occurrence of sulfamethoxazole and its two phase II metabolites acetyl-sulfamethoxazole and sulfamethoxazole glucuronide in reclaimed water during a period of one month $(n=7)$ 
CHAPTER 6

Ecological risk assessment of pharmaceuticals in reclaimed water 


\subsection{Introduction}

As described previously, some pharmaceuticals are not completely eliminated because the conventional treatments used in WWTPs appear insufficient to completely remove these specific compounds (Daughton and Ternes 1999). Consequently, these pharmaceuticals can be released through reclaimed water in variable quantities and reach surface waters, ground waters, and sediments (Zuccato et al. 2000; Wang and Gardinali 2012). Although pharmaceuticals can be degraded in the environment by biotic and/or abiotic processes and their concentrations in environmental water bodies are typically at $\mathrm{ng} / \mathrm{L}$ to low $\mu \mathrm{g} / \mathrm{L}$ level, these organic pollutants may still pose risks to aquatic species under chronic long-term exposure (Sanderson et al. 2003; Gunnarsson et al. 2008; Pounds et al. 2008; Quinn et al. 2009; Berninger and Brooks 2010). Since many aquatic species were shown to have similar physiological receptors to those the pharmaceuticals are originally intended to react with in humans (Gunnarsson et al. 2008; Berninger and Brooks 2010), the probability of ecological risks that these compounds pose to aquatic organisms cannot be ruled out.

Ecological risk assessment (ERA) is the process of analyzing and evaluating the probability of adverse ecological effects caused by environmental pollutants (USEPA 1998). In recently years, probabilistic approach which determines the probability of an exposure concentration exceeding the probability of effects has been increasingly used in ERA procedures (Solomon et al. 2001; Carriger and Rand 2008). This is because, when used correctly, it can introduce greater 
statistical confidence into risk assessment when compared to traditional hazard quotient and assessment factor approaches (Wheeler et al. 2002). However, problems associated with assessing risk of pharmaceuticals include the very large number of pharmaceuticals in use today and the availability of good toxicity benchmarks. In addition, many pharmaceuticals are biologically degraded to active metabolites that have not been fully evaluated. Therefore, on the basis of previous results (Chapter 4), five bioaccumulative pharmaceuticals including caffeine, carbamazepine, diltiazem, diphenhydramine, and ibuprofen detected in the worst case multiple exposure of reclaimed water will be fully investigated regarding acute and chronic risks to aquatic organisms.

The objective of this chapter is to assess the probabilistic risks associated with the pharmaceuticals in reclaimed water used for daily irrigation at Florida International University Biscayne Bay Campus. The procedures include risk analysis and risk characterization by comparing distributions of exposure concentrations of these pharmaceuticals with species sensitivity distribution data from well described laboratory toxicity studies.

\subsection{Methods}

\subsubsection{Exposure and toxicity data}

Concentrations of the five bioaccumulative pharmaceuticals in reclaimed water were obtained from Chapter 4 (Table 4.2), and charted in Figure 6.1. Measured concentrations were ranked from the smallest to the highest to assign a centile 
ranking $(j)$ using equation $j \times 100 /(n+1)$, where $j$ is the rank and $n$ is the total number of observations (Hall et al. 2009).

Acute (LC50/EC50) and chronic (NOEC) laboratory toxicity data for water exposure were collected from USEPA AQUIRE database and literatures (PinaVaz et al. 2000; Kim et al. 2007; Moore et al. 2008; Pounds et al. 2008; Kim et al. 2009; Quinn et al. 2009; Berninger et al. 2011; Li et al. 2011), and used to develop species sensitivity distributions (SSDs) for each pharmaceutical. For a distribution to be considered for analysis, at least four suitable species end points were used to construct SSDs (Wheeler et al. 2002; Carriger and Rand 2008). Only toxicity data characterized by a specified end point that could be clearly related to changes in population structure such as growth, reproduction and survival were used in the SSDs. Where data from multiple studies on the same species were available, the geometric mean toxicity values were used to represent the species in the distribution (Carriger and Rand 2008). End point values greater than water solubility were excluded. Chronic toxicity data for diltiazem were limited and the SSD could not be developed.

All toxicity data used for SSDs were assumed to fit a log-logistic distribution and graphical output was produced by an EPA SSD generator (http://www.epa.gov/caddis/downloads/SSD Generator V1.xlt). Log-transformed end point concentrations were plotted against the cumulative probabilities for each species. The 10th centile for each SSD was determined and chosen as the primary benchmark or threshold for effects on communities of aquatic species 
(Wheeler et al. 2002), however, the 5th centile values were also calculated for comparison purposes.

\subsubsection{Risk assessment}

Risk was assessed by comparing the overlap of the distributions of exposure concentrations and the SSDs. The estimated 90th centile of exposure concentrations were compared to the estimated 10th and 5th centile concentrations from the acute SSDs (Solomon et al. 2001). The 90th centile represents a concentration that would only be expected to occur $10 \%$ of the time and would represent episodic or pulsed exposures (Rand et al. 2010). The estimated 50th centile exposure concentrations were compared to the estimated 10th and 5th centile concentrations of the chronic SSDs (Solomon et al. 2001). The 50th centile concentration was chosen as a comparison to chronic SSDs on the basis of that it might be more representative of background concentrations as $50 \%$ of the exposures are anticipated to be above or below this level at a site (Rand et al. 2010).

When a centile from exposure distribution data was applied to SSD, the potentially affected fraction (PAF) of species was calculated (Van Beelen et al. 2001; van de Meent and Huijbregts 2005). Any exceedences above the 10th centile of a SSD were noted for acute and chronic risk. The PAF is important to understand the fraction of species that is expected to be potentially affected above its acute or chronic benchmark at a given environmental concentration. 
The calculation for PAF is shown in the following equation (Carriger and Rand 2008).

$\operatorname{PAF}(x)=\frac{1}{1+e^{-(x-\alpha) / \beta}}$

where $\alpha$ is the mean of $\log$ toxicity data, $\beta$ is equal to $\sigma \times \sqrt{3} / \pi$ while $\sigma$ is the standard deviation and $x$ is the log of the exposure concentration. As described above, $x$ was determined to be the 90th and 50th centiles of the exposure distribution for acute and chronic effects, respectively.

Next, joint probability curves (JPCs) were constructed for the pharmaceuticals that showed the highest PAF values. Joint probability curve is a function of probability of exposure and magnitude of ecological effects and used to determine the proportion of toxicity values from the SSD that are exceeded by ranges of exposure concentrations (Carriger and Rand 2008). For instance, a JPC for pharmaceutical A shows that there is a $5 \%$ probability that the exposure of A will affect $10 \%$ of the species in SSD, but another JPC for pharmaceutical B may present $15 \%$ probability that the exposure of $B$ will affect the same proportion of species in SSD. One can conclude that pharmaceutical B may pose higher risk than pharmaceutical A to aquatic species and is therefore of greater concern. Therefore, JPC provides a means of comparing relative potential risk when toxicity and exposure data are sufficiently robust. 


\subsection{Results and discussions}

Exposure concentrations of the selected pharmaceuticals in reclaimed water are shown in Figure 6.1 as boxplot. The bar closest to zero indicates the 10 th centile, a line within the box marks the median (50th centile), and the bar farthest from zero indicates the 90th centile. The 50th and 90th centiles of the exposure concentrations were determined and presented in Table 6.1. The highest 90th and 50th centile concentrations occurred for caffeine at $9.66 \mu \mathrm{g} / \mathrm{L}$ and $8.39 \mu \mathrm{g} / \mathrm{L}$, respectively. The lowest 90 th and 50th centile concentrations occurred for diltiazem at $0.183 \mu \mathrm{g} / \mathrm{L}$ and $0.159 \mu \mathrm{g} / \mathrm{L}$, respectively.

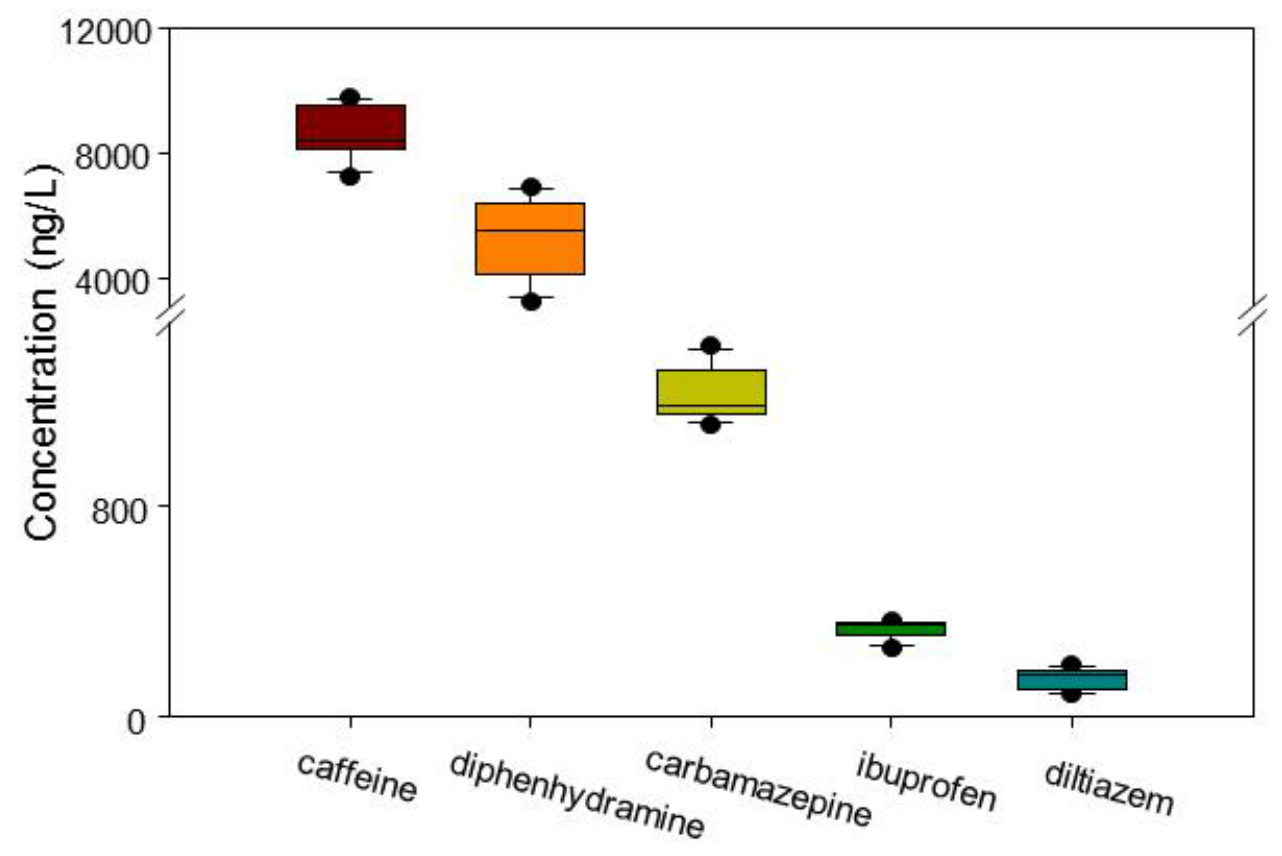

Figure 6.1 Boxplot of exposure concentrations $(n=7)$ of selected pharmaceuticals in reclaimed water 
The acute and chronic SSDs for the selected pharmaceuticals are shown in Figure 6.2 and Figure 6.3, respectively. A summary of the acute and chronic toxicity data including the 5th and 10th centiles from respective SSDs is presented in Table 6.2. Chronic SSD for diltiazem was not constructed as a result of limited chronic toxicity data. All centile concentrations from both acute and chronic toxicity data were at least one order of magnitude higher than the exposure concentrations, except for the chronic 5th and 10th centile concentrations of carbamazepine. The results indicate that carbamazepine may pose higher risk than other pharmaceuticals as it is the only compound that shows overlap between the exposure concentrations and the toxicity data. However, further probabilistic assessment is needed to confirm the risk and the results will be discussed later.

\section{Table 6.1 90th and 50th centiles of exposure concentrations}

\begin{tabular}{lcc}
\hline & $\begin{array}{c}\text { 90th centile of } \\
\text { exposure } \\
\text { concentration }(\mu \mathrm{g} / \mathrm{L})\end{array}$ & $\begin{array}{c}\text { 50th centile of } \\
\text { exposure } \\
\text { concentration }(\mu \mathrm{g} / \mathrm{L})\end{array}$ \\
\hline caffeine & 9.66 & 8.39 \\
carbamazepine & 1.37 & 1.18 \\
diltiazem & 0.183 & 0.159 \\
diphenhydramine & 6.72 & 5.53 \\
ibuprofen & 0.359 & 0.347 \\
\hline
\end{tabular}


All the chronic toxicity data were lower than the acute toxicity data indicating that the aquatic organisms under chronic exposure of these pharmaceuticals are more likely to be affected than under the acute exposures. The chronic and acute SSD centile concentrations were relatively close (within one order of magnitude) for caffeine and diphenhydramine. Carbamazepine and ibuprofen, on the other hand, had much lower chronic toxicity centile concentrations than their acute values (Table 6.2), suggesting that chronic exposure of carbamazepine or ibuprofen may pose higher risks than acute exposure.

Table 6.2 The 5th and 10th centiles of toxicity data from acute and chronic SSDs

\begin{tabular}{lcccc}
\hline & \multicolumn{2}{c}{ Acute SSD } & \multicolumn{2}{c}{ Chronic SSD } \\
& $\begin{array}{c}5 \text { th } \\
\text { centile } \\
(\mu \mathrm{g} / \mathrm{L})\end{array}$ & $\begin{array}{c}\text { 10th centile } \\
(\mu \mathrm{g} / \mathrm{L})\end{array}$ & $\begin{array}{c}5 \text { th } \\
\text { centile } \\
(\mu \mathrm{g} / \mathrm{L})\end{array}$ & $\begin{array}{c}\text { 10th centile } \\
(\mu \mathrm{g} / \mathrm{L})\end{array}$ \\
\hline caffeine & 16039 & 26070 & 4465 & 7442 \\
carbamazepine & 2253 & 3730 & 3.90 & 14.2 \\
diltiazem & 2479 & 4041 & $\mathrm{~N} / \mathrm{A}$ & $\mathrm{N} / \mathrm{A}$ \\
diphenhydramine & 438 & 598 & 139 & 336 \\
ibuprofen & 3977 & 5992 & 64 & 160 \\
\hline
\end{tabular}

$\mathrm{N} / \mathrm{A}$ indicates that data were not available for the compound. 


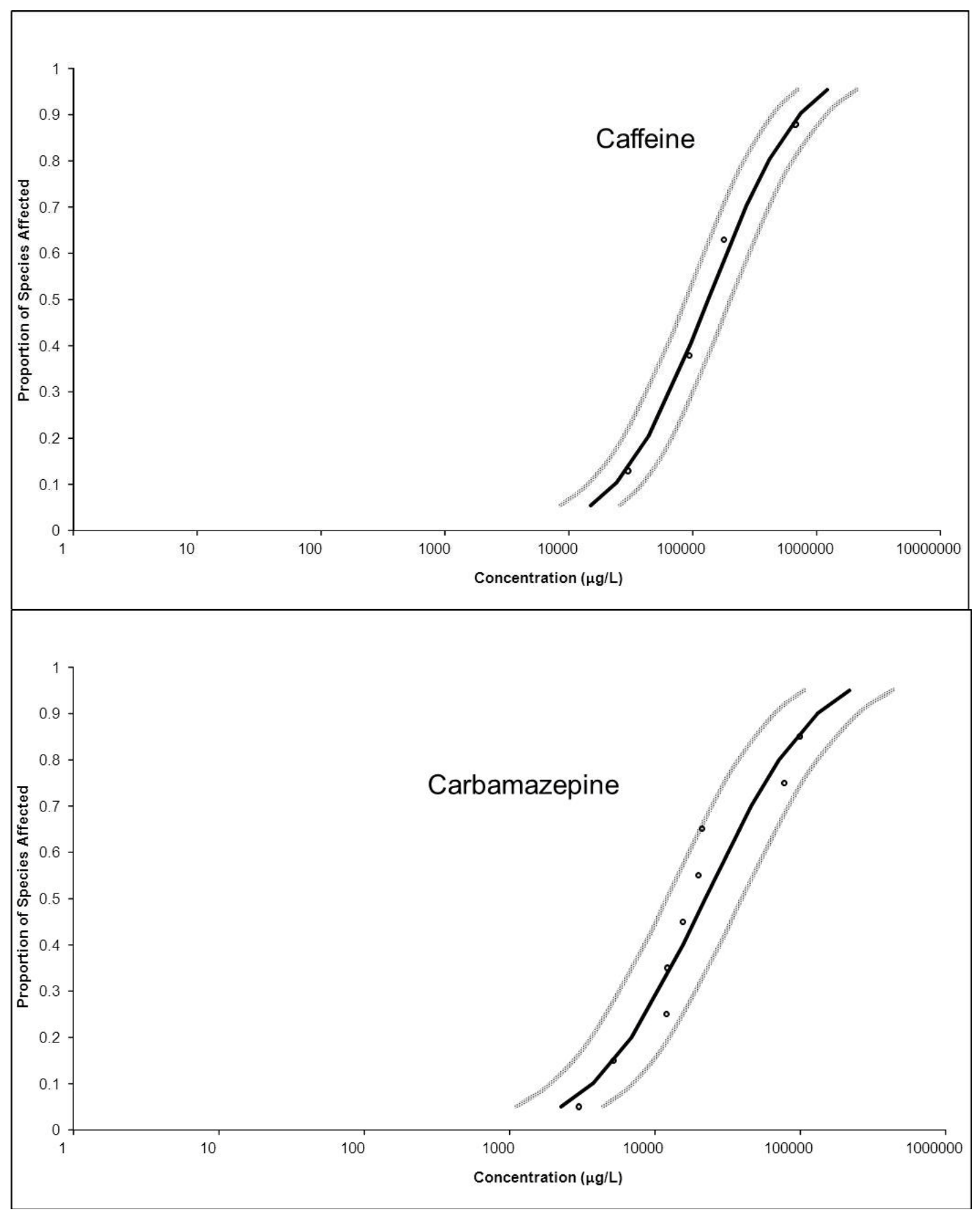




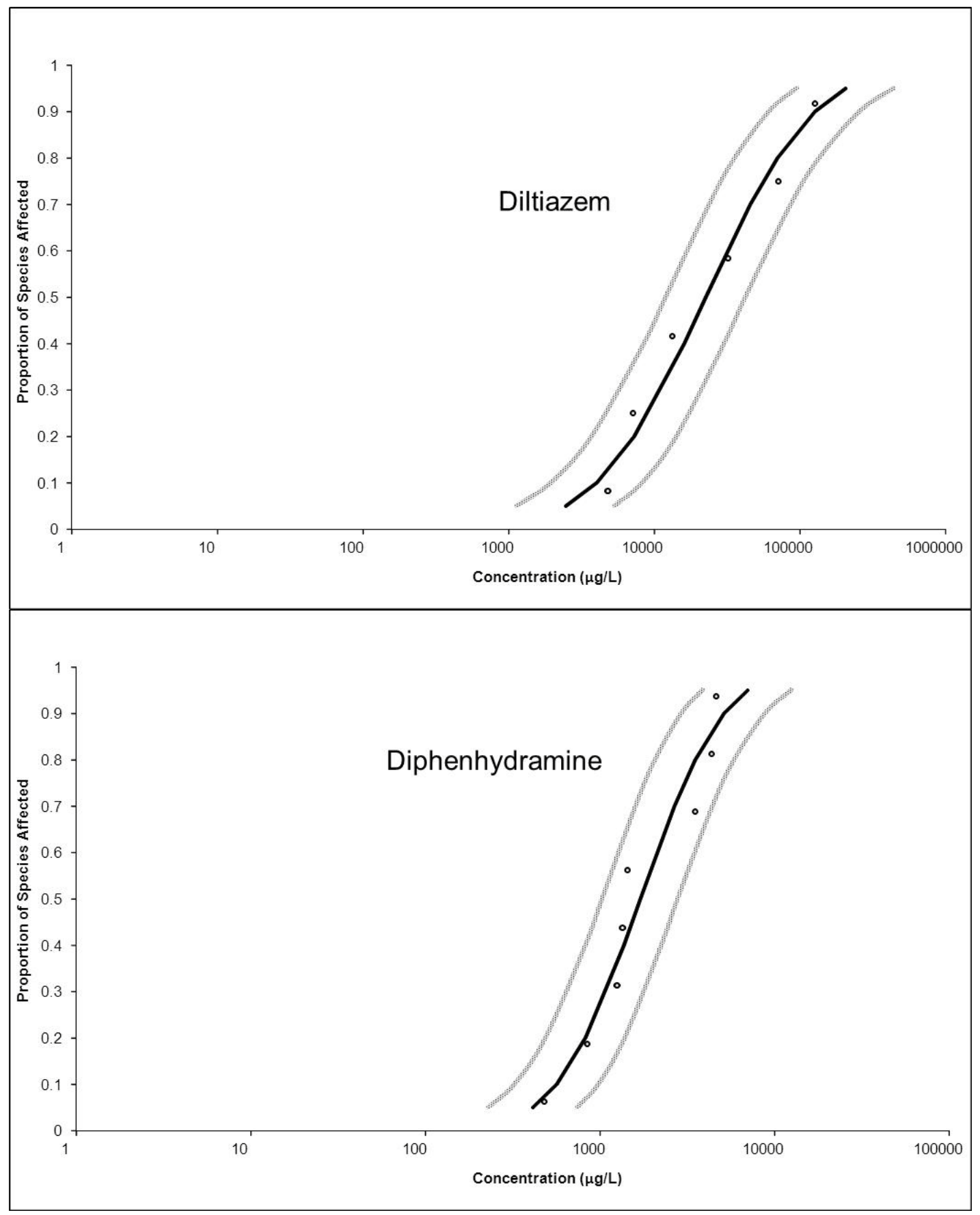




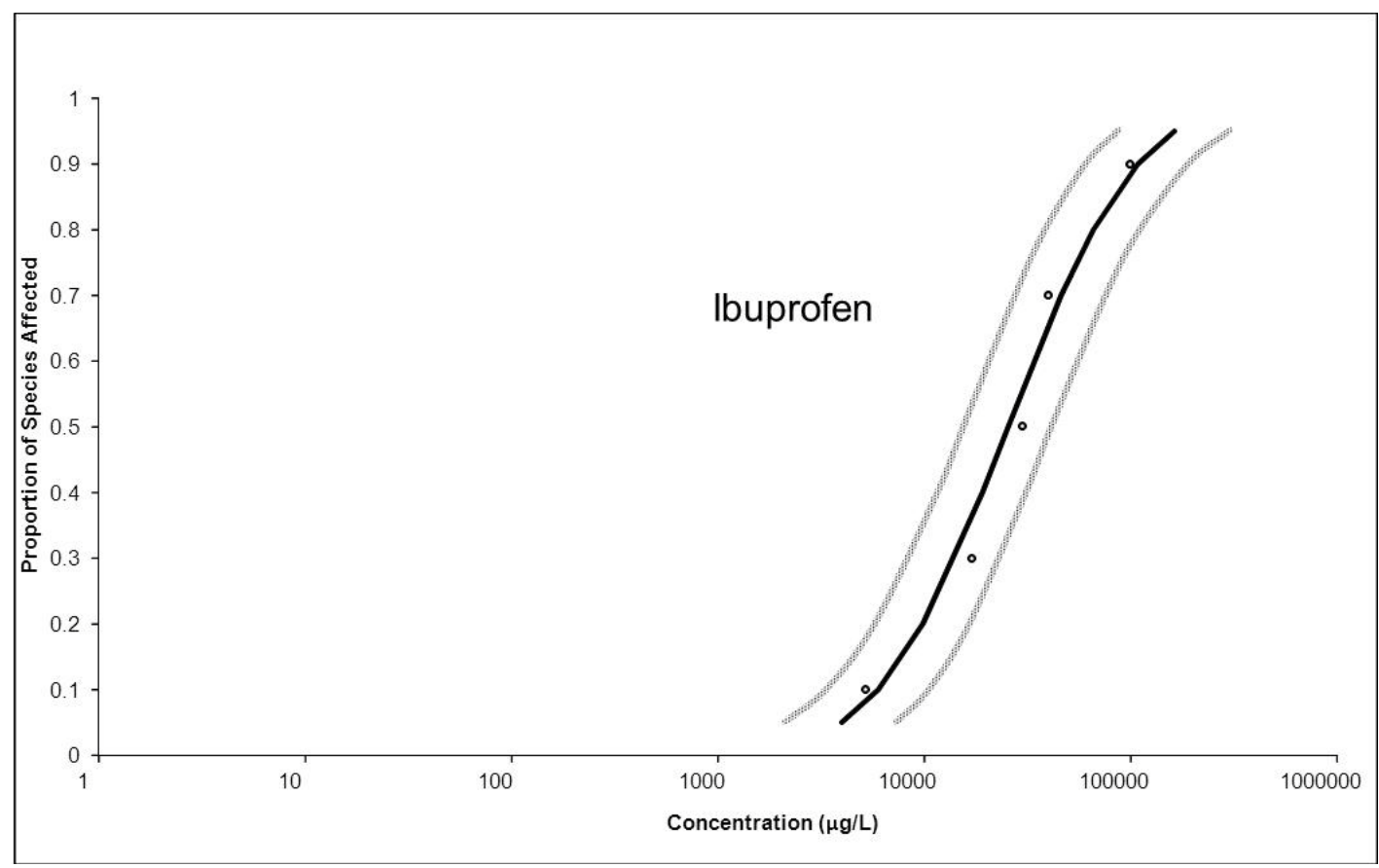

Figure 6.2 Acute species sensitivity distributions with 95\% Confidence Interval for caffeine, carbamazepine, diltiazem, diphenhydramine and ibuprofen

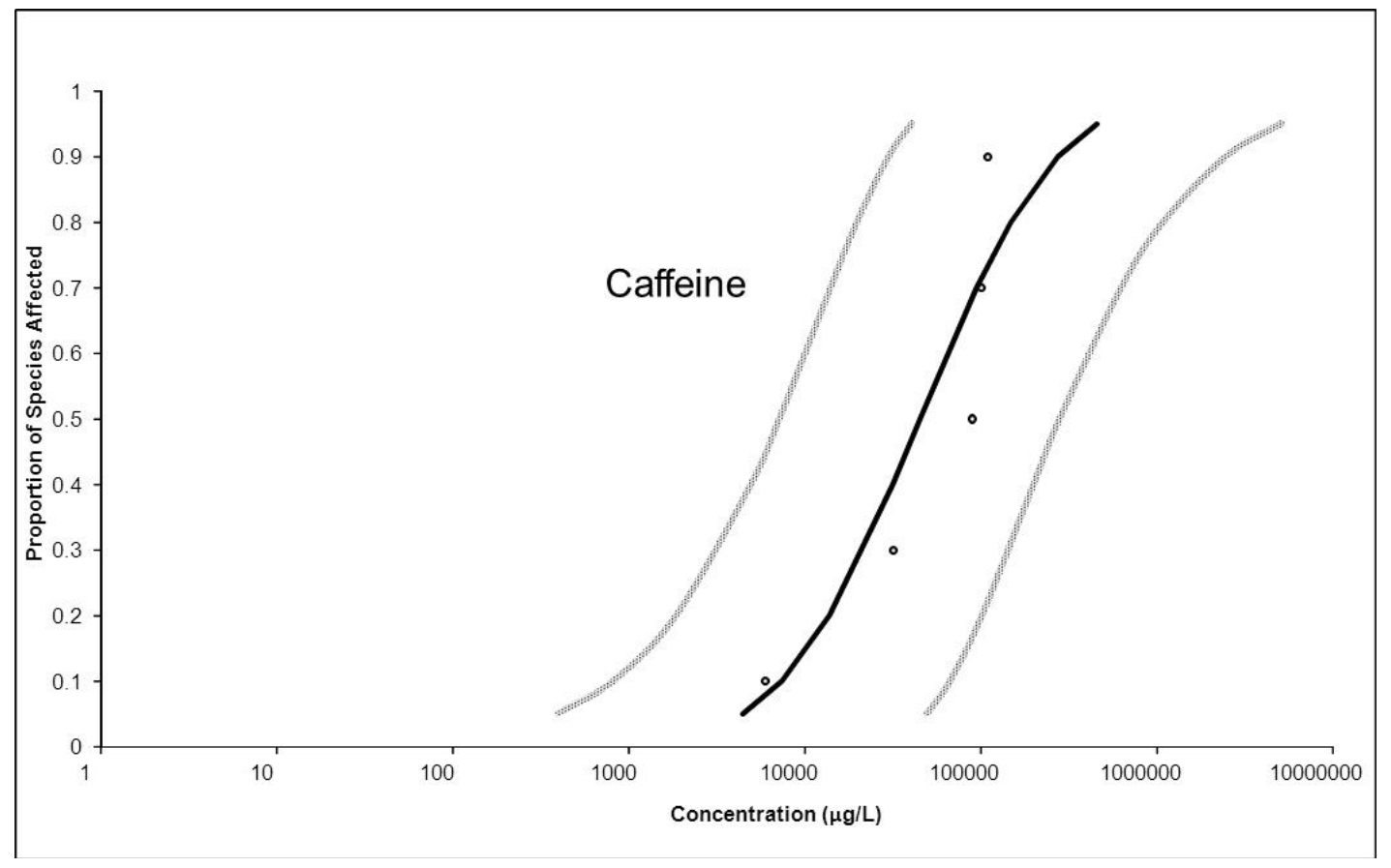




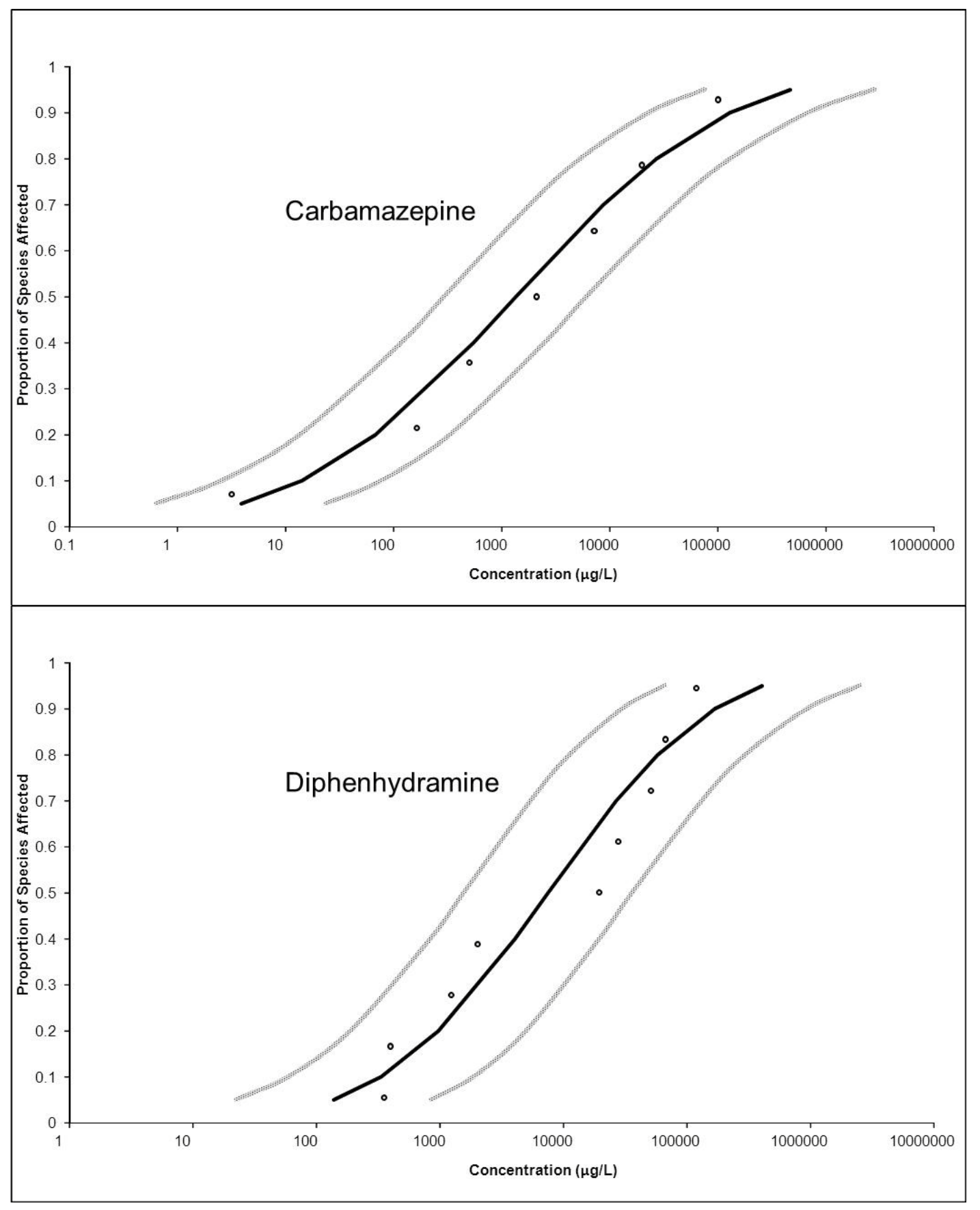




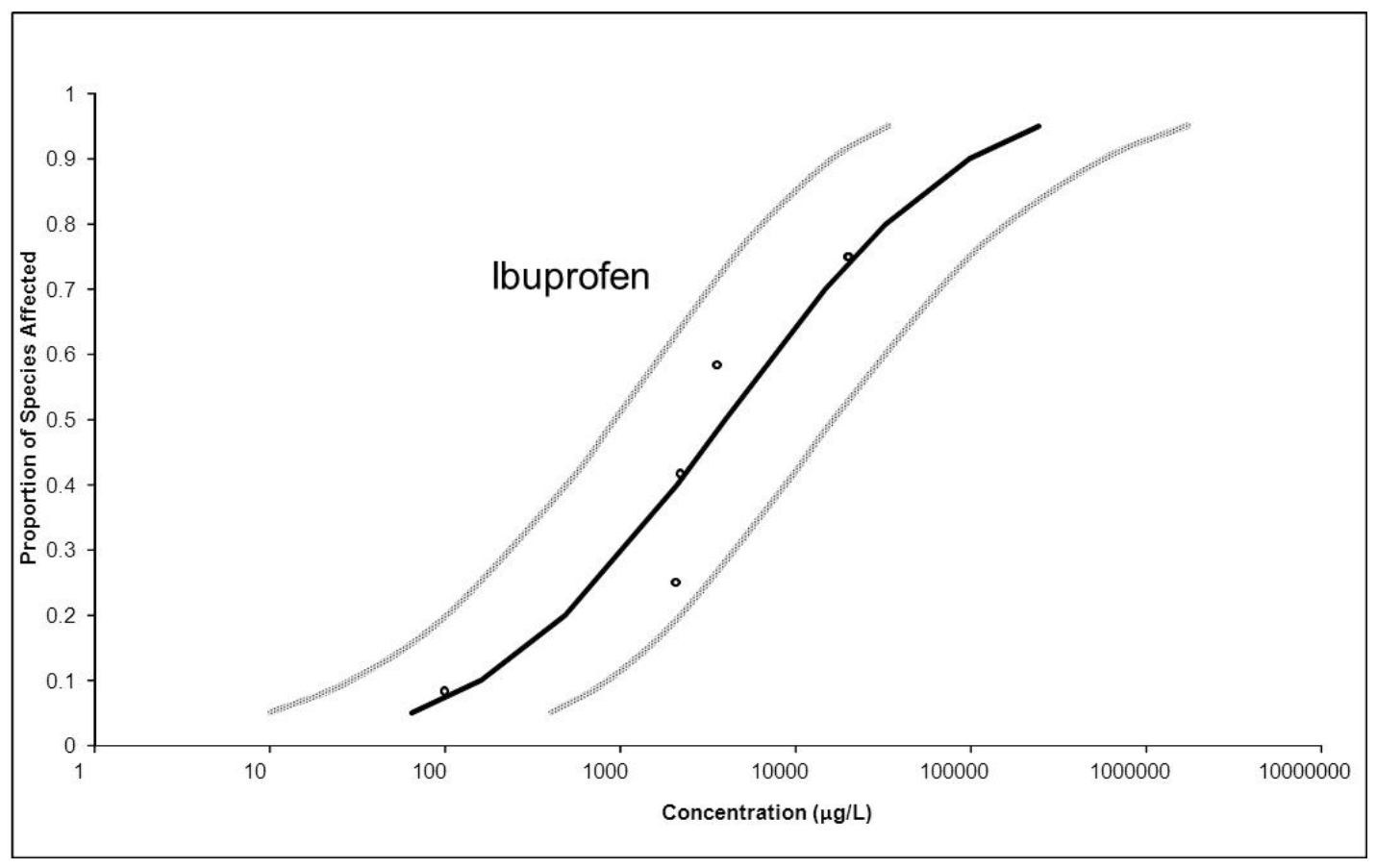

Figure 6.3 Chronic species sensitivity distributions with 95\% Confidence Interval for caffeine, carbamazepine, diphenhydramine and ibuprofen

Potentially affected fractions were calculated and shown in Table 6.3. The potentially affected fraction values for caffeine, diltiazem and ibuprofen were zero. The results indicate that there was no overlap between the exposure concentration and the SSD for each pharmaceutical. Acute and chronic PAFs for diphenhydramine were calculated at $0.1 \%$ and $0.3 \%$, respectively. Chronic PAF for carbamazepine was calculated at $2.4 \%$, but the values (less than $3 \%$ ) were generally considered low (Carriger and Rand 2008; Xing 2012). 
Table 6.3 Statistics and potentially affected fraction (PAF) for acute and chronic toxicity data for pharmaceuticals

\begin{tabular}{lcccccc}
\hline & & $x$ & $\alpha$ & $\sigma$ & $\beta$ & PAF \% \\
\hline \multirow{2}{*}{ caffeine } & Acute & 0.985 & 4.339 & 0.616 & 0.340 & 0.0 \\
carbamazepine & Chronic & 0.924 & 4.654 & 0.535 & 0.295 & 0.0 \\
& Acute & 0.137 & 4.307 & 0.522 & 0.288 & 0.0 \\
diltiazem & Chronic & 0.071 & 3.128 & 1.493 & 0.823 & 2.4 \\
& Acute & -0.737 & 4.355 & 0.564 & 0.311 & 0.0 \\
diphenhydramine & Chronic & -0.799 & $\mathrm{~N} / \mathrm{A}$ & $\mathrm{N} / \mathrm{A}$ & $\mathrm{N} / \mathrm{A}$ & $\mathrm{N} / \mathrm{A}$ \\
& Acute & 0.828 & 3.041 & 0.557 & 0.307 & 0.1 \\
ibuprofen & Chronic & 0.743 & 3.876 & 0.990 & 0.546 & 0.3 \\
& Acute & -0.445 & 4.406 & 0.474 & 0.262 & 0.0 \\
& Chronic & -0.460 & 3.468 & 0.847 & 0.467 & 0.0 \\
\hline
\end{tabular}

N/A indicates that data were not available for the compound.

Zero indicates that there was no overlap between the exposure concentration and the SSD.

On the basis of chronic PAF values, carbamazepine could be considered as a compound of concern. Therefore, a JPC was developed and shown in Figure 6.4. It can be seen that the curve was extremely close to the axes. As a matter of fact, the probability for $5 \%$ of species affected was zero, indicating a very small probability of adverse effects. 


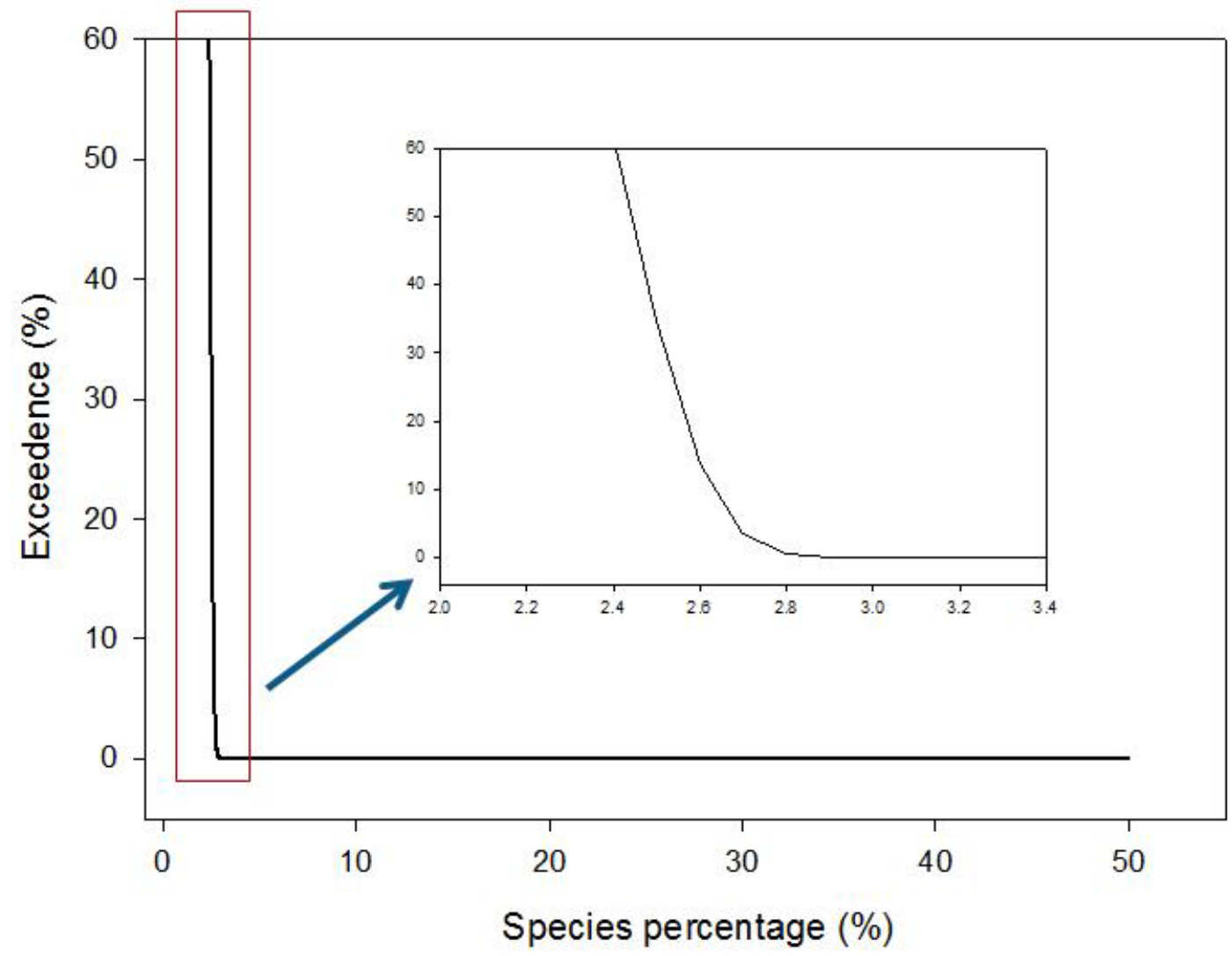

Figure 6.4 Joint probability curve for the exposure concentration and chronic toxicity data of carbamazepine

\subsection{Conclusion}

In this chapter, five bioaccumulative pharmaceuticals including caffeine, carbamazepine, diltiazem, diphenhydramine and ibuprofen detected in reclaimed water were investigated to assess the potential acute and chronic risks to aquatic organisms. The results showed that aquatic organisms under chronic exposure of these pharmaceuticals are more likely to be affected than under acute exposure. In addition, the joint probability curve for the exposure and chronic toxicity data of carbamazepine indicated a quantifiably low potential risk even 
under the worst case exposure to reclaimed water. Given the dilution factors that affect environmental releases, the risk of exposure to carbamazepine will be even more decreased. 


\section{CONCLUSIONS}

The overall objectives of this research were to develop analytical methods for the determination of trace level pharmaceuticals and metabolites as emerging environmental contaminants using the-state-of-art mass spectrometry in different matrices, and to gain knowledge about the occurrence, distribution and variance of these pharmaceutically active compounds in the South Florida environment as well as the information regarding bioconcentration factors and pharmacokinetics in aquatic species being affected by wastewater under various exposure scenarios.

As a result, two protocols were successfully developed for targeted analysis and unknown phase II metabolites screening. A specific and sensitive triple quadruple mass spectrometer was used in the targeted analysis of pharmaceuticals in mosquito fish and surface water directly affected by reclaimed water. A high resolution Orbitrap mass spectrometer was used in the phase II metabolites screening.

After a comprehensive evaluation of a large number of environmental samples, it was confirmed that a number of pharmaceuticals at concentrations that could range up to $\mu \mathrm{g} / \mathrm{L}$ were routinely introduced into the environment from a typical secondary wastewater treatment plant. Compounds such as caffeine, diphenhydramine and carbamazepine were consistently detected in reclaimed water and surface water from a fresh water pond directly affected by reclaimed

water. Bioaccumulation factors for caffeine, diphenhydramine and 
carbamazepine in mosquito fish living in this pond were also calculated and found at $29 \pm 26,821 \pm 422$ and $108 \pm 144$, respectively.

In order to understand the uptake and depuration as well as bioconcentration factors (BCFs) under the worst-case conditions, mosquito fish were exposed to reclaimed water under static-renewal for 7 days, followed by a 14-day depuration phase in clean water. The results showed that some pharmaceuticals such as diphenhydramine could be accumulated in fish up to $82 \mathrm{ng} / \mathrm{g}$ while some pharmaceuticals such as caffeine will rather equilibrate with the surrounding waters and would persist for a period of several days. The study also showed that a number of other compounds detected in rather high concentrations (e.g. diclofenac and gemfibrozil) did not accumulate in fish despite having moderately high Log Kow values implying that other mechanisms may have played a role in keeping them in solution. Because many aquatic species have similar physiological receptors to those originally targeted for pharmacological effects in humans, non-target species under chronic exposure may show potential adverse effects.

Next, the combination of the power of high resolution Orbitrap mass spectrometry and metabolic profiling software was successfully applied for the analysis of pharmaceutical metabolites in reclaimed water. Sulfamethoxazole and its two phase II metabolites, acetyl-sulfamethoxazole and sulfamethoxazole glucuronide, were successfully identified in reclaimed water using this technique. It was also illustrated that it was helpful to use characteristic ions and MS/MS spectra to 
identify a compound when the standard is not available. Preliminary results showed that the method could be easily adapted to the analysis in various matrices such as sewage extracts, surface water and drinking water. The resulting data can be used to evaluate the performance of reclaimed water treatment procedures, also the fate of pharmaceuticals in wastewater treatment plants. In the case of sulfamethoxazole, the amount of acetyl-sulfamethoxazole and sulfamethoxazole glucuronide needs to be considered in order to correctly assess the fate of sulfamethoxazole as they represent the major source of sulfamethoxazole in the reclaimed water.

Finally, five bioaccumulative pharmaceuticals including caffeine, carbamazepine, diltiazem, diphenhydramine and ibuprofen detected in reclaimed water were investigated regarding the acute and chronic risks to aquatic organisms. The results showed that aquatic organisms under chronic exposure of carbamazepine are more likely to be affected than the exposures of any other selected pharmaceuticals. In addition, the joint probability curve for the exposure and chronic toxicity data of carbamazepine indicated a low potential risk of carbamazepine even under the worst case exposure scenario. However, given the dilution factors that affect environmental releases, the risk of exposure to carbamazepine will be drastically decreased. 


\section{REFERENCES}

"Commission Decision (2002/657/EC) of 12 August 2002 Implementing Council Directive 96/23/EC concerning the performance of analytical methods and the interpretation of results." Official Journal of the European Communities L221: 836.

"KABAM Version 1.0 (Kow (based) Aquatic BioAccumulation Model) www.epa.gov/oppefed1/models/water/kabam/kabam v1 0.xls."

(1986). "Definition and procedure for the determination of the method detection limit. ." Code of Federal Regulations Title 40: Section 136, Appendix B.

(2009). "EPA pilot study of PPCPs in fish tissue. http://www.epa.gov/waterscience/ppcp/files/fish-pilot.pdf (accessed November 2009).".

Andrisano, V., et al. (2001). "Photostability and phototoxicity studies on diltiazem." J Pharm Biomed Anal 25(3-4): 589-97.

Balmer, M. E., et al. (2004). "Occurrence of methyl triclosan, a transformation product of the bactericide triclosan, in fish from various lakes in Switzerland." Environ Sci Technol 38(2): 390-5.

Barron, M. G., et al. (1990). "Pharmacokinetic modeling in aquatic animals I. Models and concepts." Aquatic Toxicology 18(2): 61-85.

Bedner, M. and W. A. MacCrehan (2006). "Transformation of acetaminophen by chlorination produces the toxicants 1,4-benzoquinone and $\mathrm{N}$-acetyl-pbenzoquinone imine." Environ Sci Technol 40(2): 516-22.

Berninger, J. P. and B. W. Brooks (2010). "Leveraging mammalian pharmaceutical toxicology and pharmacology data to predict chronic fish responses to pharmaceuticals." Toxicol Lett 193(1): 69-78.

Berninger, J. P., et al. (2011). "Effects of the antihistamine diphenhydramine on selected aquatic organisms." Environ Toxicol Chem 30(9): 2065-72.

Berrada, H., et al. (2008). "Determination of macrolide antibiotics in meat and fish using pressurized liquid extraction and liquid chromatography-mass spectrometry." J Chromatogr A 1208(1-2): 83-9.

Berthiller, F., et al. (2006). "Liquid chromatography coupled to tandem mass spectrometry (LC-MS/MS) determination of phase II metabolites of the mycotoxin 
zearalenone in the model plant Arabidopsis thaliana." Food Addit Contam 23(11): 1194-200.

Blanco, S., et al. (2004). "Experimental Study on the Diet of Mosquitofish (Gambusia holbrooki) under Different Ecological Conditions in a Shallow Lake." International Review of Hydrobiology 89(3): 250-262.

Bonvin, F., et al. (2012). "Direct Photolysis of Human Metabolites of the Antibiotic Sulfamethoxazole: Evidence for Abiotic Back-Transformation." Environ Sci Technol.

Boyd, G. R., et al. (2003). "Pharmaceuticals and personal care products (PPCPs) in surface and treated waters of Louisiana, USA and Ontario, Canada." Sci Total Environ 311(1-3): 135-49.

Brooks, B. W., et al. (2005). "Determination of select antidepressants in fish from an effluent-dominated stream." Environ Toxicol Chem 24(2): 464-9.

Brooks, B. W., et al. (2006). "Water Quality of Effluent-dominated Ecosystems: Ecotoxicological, Hydrological, and Management Considerations." Hydrobiologia 556(1): 365-379.

Buser, H. R., et al. (2006). "Occurrence of UV filters 4-methylbenzylidene camphor and octocrylene in fish from various Swiss rivers with inputs from wastewater treatment plants." Environ Sci Technol 40(5): 1427-31.

Calza, P., et al. (2012). "Identification of the unknown transformation products derived from lincomycin using LC-HRMS technique." J Mass Spectrom 47(6): 751-9.

Carriger, J. F. and G. M. Rand (2008). "Aquatic risk assessment of pesticides in surface waters in and adjacent to the Everglades and Biscayne National Parks: II. Probabilistic analyses." Ecotoxicology 17(7): 680-96.

Celiz, M. D., et al. (2009). "Pharmaceutical metabolites in the environment: analytical challenges and ecological risks." Environ Toxicol Chem 28(12): 247384.

Chen, H., et al. (2005). "Liquid chromatography-tandem mass spectrometry analysis of anisodamine and its phase I and II metabolites in rat urine." $\underline{\mathrm{J}}$ Chromatogr B Analyt Technol Biomed Life Sci 824(1-2): 21-9.

Chu, S. and C. D. Metcalfe (2007). "Analysis of paroxetine, fluoxetine and norfluoxetine in fish tissues using pressurized liquid extraction, mixed mode solid 
phase extraction cleanup and liquid chromatography-tandem mass spectrometry." J Chromatogr A 1163(1-2): 112-8.

Danielson, P. B. (2002). "The cytochrome P450 superfamily: biochemistry, evolution and drug metabolism in humans." Curr Drug Metab 3(6): 561-97.

Datta, S., et al. (2002). "A simple method for the determination of trace levels of alkylphenolic compounds in fish tissue using pressurized fluid extraction, solid phase cleanup, and high-performance liquid chromatography fluorescence detection." J Agric Food Chem 50(6): 1350-4.

Daughton, C. G. and T. A. Ternes (1999). "Pharmaceuticals and personal care products in the environment: agents of subtle change?" Environ Health Perspect 107 Suppl 6: 907-38.

Davis, J. G., et al. (2006). "Antibiotic Transport via Runoff and Soil Loss." 므 Environ Qual 35(6): 2250-60.

Draisci, R., et al. (1998). "Evaluation of musk contamination of freshwater fish in Italy by accelerated solvent extraction and gas chromatography with mass spectrometric detection." J Chromatogr A 814(1-2): 187-97.

Duedahl-Olesen, L., et al. (2005). "Synthetic musk fragrances in trout from Danish fish farms and human milk." Chemosphere 61(3): 422-31.

Duffy, E. M. and W. L. Jorgensen (2000). "Prediction of Properties from Simulations: Free Energies of Solvation in Hexadecane, Octanol, and Water." J. Am. Chem. Soc. 122: 2878-2888.

Eichhorn, P., et al. (2005). "Application of ion trap-MS with H/D exchange and QqTOF-MS in the identification of microbial degradates of trimethoprim in nitrifying activated sludge." Anal Chem 77(13): 4176-84.

EPA (2009). "EPA pilot study of PPCPs in fish tissue. http://www.epa.gov/waterscience/ppcp/files/fish-pilot.pdf (accessed November 2009).".

EPA (2009). "Expanded Investigations of Pharmaceuticals in Fish Tissue." U.S. Environmental Protection Agency. http://water.epa.gov/scitech/swguidance/ppcp/fish-expand.cfm. Accessed 1 Oct $\underline{2010}$.

Ferrer, I. and E. M. Thurman (2010). "Identification of a new antidepressant and its glucuronide metabolite in water samples using liquid 
chromatography/quadrupole time-of-flight mass spectrometry." Anal Chem 82(19): 8161-8.

Ferrer, I. and E. M. Thurman (2012). "Analysis of 100 pharmaceuticals and their degradates in water samples by liquid chromatography/quadrupole time-of-flight mass spectrometry." J Chromatogr A 1259: 148-57.

Fick, J., et al. (2010). "Therapeutic levels of levonorgestrel detected in blood plasma of fish: results from screening rainbow trout exposed to treated sewage effluents." Environ Sci Technol 44(7): 2661-6.

Fick, J., et al. (2010). "Predicted critical environmental concentrations for 500 pharmaceuticals." Regul Toxicol Pharmacol 58(3): 516-23.

Garcia, A., et al. (2009). "On-line solid-phase extraction of large-volume injections coupled to liquid chromatography-tandem mass spectrometry for the quantitation and confirmation of 14 selected trace organic contaminants in drinking and surface water." J Chromatogr A 1216(48): 8518-27.

Gobel, A., et al. (2004). "Trace determination of macrolide and sulfonamide antimicrobials, a human sulfonamide metabolite, and trimethoprim in wastewater using liquid chromatography coupled to electrospray tandem mass spectrometry." Anal Chem 76(16): 4756-64.

Gomez-Martinez, L. E. (2011). "Disposition kinetics of caffeine and paraxanthine in Nile tilapia (Oreochromis niloticus): characterization of the main metabolites." Arch Environ Contam Toxicol 60(4): 654-64.

Gossett, R., et al. (1983). "Predicting the bioaccumulation of organic compounds in marine organisms using octanol/water partition coefficients." Marine Pollution Bulletine 14(10): 387-392.

$\mathrm{Gu}$, J., et al. (2003). "Liver-specific deletion of the NADPH-cytochrome P450 reductase gene: impact on plasma cholesterol homeostasis and the function and regulation of microsomal cytochrome P450 and heme oxygenase." J Biol Chem 278(28): 25895-901.

Guengerich, F. P. and W. W. Johnson (1997). "Kinetics of ferric cytochrome P450 reduction by NADPH-cytochrome P450 reductase: rapid reduction in the absence of substrate and variations among cytochrome P450 systems." Biochemistry 36(48): 14741-50.

Gunnarsson, L., et al. (2008). "Evolutionary conservation of human drug targets in organisms used for environmental risk assessments." Environ Sci Technol 42(15): 5807-13. 
Haglund, P., et al. (2007). "Shape-selective extraction of PCBs and dioxins from fish and fish oil using in-cell carbon fractionation pressurized liquid extraction." Anal Chem 79(7): 2945-51.

Hall, L. W., Jr., et al. (2009). "Ecological risk of Irgarol 1051 and its major metabolite in coastal California marinas and reference areas." Mar Pollut Bull 58(5): 702-10.

Heberer, T. (2002). "Occurrence, fate, and removal of pharmaceutical residues in the aquatic environment: a review of recent research data." Toxicol Lett 131(1-2): 5-17.

Hendriks, A. J., et al. (2001). "The power of size. 1. Rate constants and equilibrium ratios for accumulation of organic substances related to octanol-water partition ratio and species weight." Environ Toxicol Chem 20(7): 1399-420.

Hirsch, R., et al. (1999). "Occurrence of antibiotics in the aquatic environment." Sci Total Environ 225(1-2): 109-18.

Hoang, T. C., et al. (2011). "Bioconcentration and depuration of endosulfan sulfate in mosquito fish (Gambusia affinis)." Chemosphere 84(5): 538-43.

Holcapek, M., et al. (2008). "High-performance liquid chromatography-tandem mass spectrometry in the identification and determination of phase I and phase II drug metabolites." Anal Bioanal Chem 391(1): 59-78.

Ibanez, M., et al. (2012). "Importance of MS selectivity and chromatographic separation in LC-MS/MS-based methods when investigating pharmaceutical metabolites in water. Dipyrone as a case of study." J Mass Spectrom 47(8): 1040-6.

Kim, J. W., et al. (2009). "Acute toxicity of pharmaceutical and personal care products on freshwater crustacean (Thamnocephalus platyurus) and fish (Oryzias latipes)." J Toxicol Sci 34(2): 227-32.

Kim, Y., et al. (2007). "Aquatic toxicity of acetaminophen, carbamazepine, cimetidine, diltiazem and six major sulfonamides, and their potential ecological risks in Korea." Environ Int 33(3): 370-5.

Kwon, J. W. and K. L. Armbrust (2005). "Degradation of citalopram by simulated sunlight." Environ Toxicol Chem 24(7): 1618-23.

Kwon, J. W. and K. L. Armbrust (2006). "Laboratory persistence and fate of fluoxetine in aquatic environments." Environ Toxicol Chem 25(10): 2561-8. 
Lajeunesse, A., et al. (2008). "Determination of basic antidepressants and their $\mathrm{N}$-desmethyl metabolites in raw sewage and wastewater using solid-phase extraction and liquid chromatography-tandem mass spectrometry." Anal Chem 80(14): 5325-33.

$\mathrm{Li}$, Z. H., et al. (2011). "Acute toxicity of carbamazepine to juvenile rainbow trout (Oncorhynchus mykiss): effects on antioxidant responses, hematological parameters and hepatic EROD." Ecotoxicol Environ Saf 74(3): 319-27.

Lim, H. K., et al. (2007). "Metabolite identification by data-dependent accurate mass spectrometric analysis at resolving power of 60,000 in external calibration mode using an LTQ/Orbitrap." Rapid Commun Mass Spectrom 21(12): 1821-32.

Llorca, M., et al. (2009). "Development and validation of a pressurized liquid extraction liquid chromatography-tandem mass spectrometry method for perfluorinated compounds determination in fish." J Chromatogr A 1216(43): 7195-204.

Losada, S., et al. (2009). "Selective pressurized liquid extraction of polybrominated diphenyl ethers in fish." Talanta 80(2): 839-45.

Lu, A. Y., et al. (1969). "Resolution of the cytochrome P-450-containing omegahydroxylation system of liver microsomes into three components." $\mathrm{J}$ Biol Chem 244(13): 3714-21.

Lund, M., et al. (2009). "Extraction of polycyclic aromatic hydrocarbons from smoked fish using pressurized liquid extraction with integrated fat removal." Talanta 79(1): 10-5.

Meredith-Williams, M., et al. (2012). "Uptake and depuration of pharmaceuticals in aquatic invertebrates." Environ Pollut 165: 250-8.

Miao, X. S. and C. D. Metcalfe (2003). "Determination of carbamazepine and its metabolites in aqueous samples using liquid chromatography-electrospray tandem mass spectrometry." Anal Chem 75(15): 3731-8.

Miao, X. S., et al. (2005). "Carbamazepine and its metabolites in wastewater and in biosolids in a municipal wastewater treatment plant." Environ Sci Technol 39(19): 7469-75.

Moore, M. T., et al. (2008). "Assessing caffeine as an emerging environmental concern using conventional approaches." Arch Environ Contam Toxicol 54(1): $31-5$. 
Mottaleb, M. A., et al. (2009). "Gas chromatography-mass spectrometry screening methods for select UV filters, synthetic musks, alkylphenols, an antimicrobial agent, and an insect repellent in fish." J Chromatogr A 1216(5): 815-23.

Murphy, P. J. (2001). "Xenobiotic metabolism: a look from the past to the future." Drug Metab Dispos 29(6): 779-80.

Nakamura, Y., et al. (2008). "The effects of pH on fluoxetine in Japanese medaka (Oryzias latipes): acute toxicity in fish larvae and bioaccumulation in juvenile fish." Chemosphere 70(5): 865-73.

Nelis, H. J., De Leenheer,A. P., (1983). "Isocratic nonaqueous reversed-phase liquid chromatography of carotenoids." Anal Chem 55(2): 270-275.

Owen, S. F., et al. (2007). "Comparative physiology, pharmacology and toxicology of beta-blockers: mammals versus fish." Aquat Toxicol 82(3): 145-62.

Paterson, G. and C. D. Metcalfe (2008). "Uptake and depuration of the antidepressant fluoxetine by the Japanese medaka (Oryzias latipes)." Chemosphere 74(1): 125-30.

Peterman, S. M., et al. (2006). "Application of a linear ion trap/orbitrap mass spectrometer in metabolite characterization studies: examination of the human liver microsomal metabolism of the non-tricyclic anti-depressant nefazodone using data-dependent accurate mass measurements." J Am Soc Mass Spectrom 17(3): 363-75.

Petrovic, M., et al. (2006). "Multi-residue analysis of pharmaceuticals in wastewater by ultra-performance liquid chromatography-quadrupole-time-of-flight mass spectrometry." J Chromatogr A 1124(1-2): 68-81.

Pickhardt, P. C., et al. (2006). "Contrasting uptake routes and tissue distributions of inorganic and methylmercury in mosquitofish (Gambusia affinis) and redear sunfish (Lepomis microlophus)." Environ Toxicol Chem 25(8): 2132-42.

Pina-Vaz, C., et al. (2000). "Antifungal activity of ibuprofen alone and in combination with fluconazole against Candida species." J Med Microbiol 49(9): $831-40$.

Pounds, N., et al. (2008). "Acute and chronic effects of ibuprofen in the mollusc Planorbis carinatus (Gastropoda: Planorbidae)." Ecotoxicol Environ Saf 70(1): 47-52. 
Qiang, Z. and C. Adams (2004). "Potentiometric determination of acid dissociation constants (pKa) for human and veterinary antibiotics." Water Res 38(12): 2874-90.

Quinn, B., et al. (2009). "Evaluation of the acute, chronic and teratogenic effects of a mixture of eleven pharmaceuticals on the cnidarian, Hydra attenuata." Sci Total Environ 407(3): 1072-9.

Ramirez, A. J., et al. (2009). "Occurrence of pharmaceuticals and personal care products (PPCPs) in fish: Results of a national pilot study in the U.S." Environ Toxicol Chem 28(12): 2587-2597.

Ramirez, A. J., et al. (2007). "Analysis of pharmaceuticals in fish using liquid chromatography-tandem mass spectrometry." Anal Chem 79(8): 3155-63.

Rand, G. M., et al. (2010). "Endosulfan and its metabolite, endosulfan sulfate, in freshwater ecosystems of South Florida: a probabilistic aquatic ecological risk assessment." Ecotoxicology 19(5): 879-900.

Renew, J. E. and C. H. Huang (2004). "Simultaneous determination of fluoroquinolone, sulfonamide, and trimethoprim antibiotics in wastewater using tandem solid phase extraction and liquid chromatography-electrospray mass spectrometry." J Chromatogr A 1042(1-2): 113-21.

Rudel, H., et al. (2006). "Retrospective monitoring of synthetic musk compounds in aquatic biota from German rivers and coastal areas." J Environ Monit 8(8): 812-23.

Ruhoy, I. S. and C. G. Daughton (2008). "Beyond the medicine cabinet: an analysis of where and why medications accumulate." Environ Int 34(8): 1157-69.

Sacher, F., et al. (2001). "Pharmaceuticals in groundwaters analytical methods and results of a monitoring program in Baden-Wurttemberg, Germany." ㄴ Chromatogr A 938(1-2): 199-210.

Sanderson, H., et al. (2003). "Probabilistic hazard assessment of environmentally occurring pharmaceuticals toxicity to fish, daphnids and algae by ECOSAR screening." Toxicol Lett 144(3): 383-95.

Schultz, M. M., et al. (2010). "Antidepressant Pharmaceuticals in Two U.S. Effluent-Impacted Streams: Occurrence and Fate in Water and Sediment, and Selective Uptake in Fish Neural Tissue." Environ Sci Technol. 
Schultz, M. M., et al. (2011). "Selective uptake and biological consequences of environmentally relevant antidepressant pharmaceutical exposures on male fathead minnows." Aquat Toxicol 104(1-2): 38-47.

Schultzen, O. and B. Naunyn (1867). "The behavior of benzene-derived hydrocarbons in the animal organism." duBois-Reymonds Arch Anat Physiol: 349.

Segura, P. A., Gagnon, Christian., Sebastien Sauve, (2007). "A fully automated on-line preconcentration and liquid chromatography-tandem mass spectrometry method for the analysis of anti-infectives in wastewaters." Anal Chim Acta 604(2): $147-57$.

Segura, P. A., Gagnon, Christian., Sebastien Sauve, (2009). "Application of turbulent flow chromatography load columns for the on-line analysis of antiinfectives in wastewaters." chromatographia 70: 239-245.

Smith, S., et al. (2009). "Simultaneous screening and confirmation of multiple classes of drug residues in fish by liquid chromatography-ion trap mass spectrometry." J Chromatogr A 1216(46): 8224-32.

Solomon, K. R., et al. (2001). "Probabilistic risk assessment of cotton pyrethroids: I. Distributional analyses of laboratory aquatic toxicity data." Environ Toxicol Chem 20(3): 652-9.

Stackelberg, P. E., et al. (2007). "Efficiency of conventional drinking-watertreatment processes in removal of pharmaceuticals and other organic compounds." Sci Total Environ 377(2-3): 255-72.

Tang, H. P., et al. (2006). "High-throughput screening for multi-class veterinary drug residues in animal muscle using liquid chromatography/tandem mass spectrometry with on-line solid-phase extraction." Rapid Commun Mass Spectrom 20(17): 2565-72.

Tavazzi, S., et al. (2002). "Accelerated solvent extraction then liquid chromatography coupled with mass spectrometry for determination of 4-toctylphenol, 4-nonylphenols, and bisphenol A in fish liver." Chromatographia 56: 463-467.

Thurman, E. M. and I. Ferrer (2012). "Liquid chromatography/quadrupole-time-offlight mass spectrometry with metabolic profiling of human urine as a tool for environmental analysis of dextromethorphan." J Chromatogr A 1259: 158-66.

USEPA (1998). "Guideline for ecological risk assessment. EPA/630/R-95/002F. USEPA, Washington,DC,USA." 
Van Beelen, P., et al. (2001). "The relation between extrapolated risk, expressed as potentially affected fraction, and community effects, expressed as pollutioninduced community tolerance." Environ Toxicol Chem 20(5): 1133-40.

van de Meent, D. and M. A. Huijbregts (2005). "Calculating life-cycle assessment effect factors from potentially affected fraction-based ecotoxicological response functions." Environ Toxicol Chem 24(6): 1573-8.

Vanderford, B. J., et al. (2003). "Analysis of endocrine disruptors, pharmaceuticals, and personal care products in water using liquid chromatography/tandem mass spectrometry." Anal Chem 75(22): 6265-74.

Vernouillet, G., et al. (2010). "Toxic effects and bioaccumulation of carbamazepine evaluated by biomarkers measured in organisms of different trophic levels." Chemosphere 80(9): 1062-8.

Wahlen, R. (2004). "Fast and accurate determination of arsenobetaine in fish tissues using accelerated solvent extraction and HPLC-ICP-MS determination." $\underline{\mathrm{J}}$ Chromatogr Sci 42(4): 217-22.

Wang, C. and P. R. Gardinali (2012). "Comparison of multiple API techniques for the simultaneous detection of microconstituents in water by on-line SPE-LCMS/MS." J Mass Spectrom 47(10): 1255-68.

Wang, J. and P. R. Gardinali (2012). "Analysis of selected pharmaceuticals in fish and the fresh water bodies directly affected by reclaimed water using liquid chromatography-tandem mass spectrometry." Anal Bioanal Chem 404(9): 271120.

Wheeler, J. R., et al. (2002). "Species sensitivity distributions: data and model choice." Mar Pollut Bull 45(1-12): 192-202.

Williams, R. (1947). "Detoxication Mechanisms." John Wiley and Sons, Inc, New York.

Wohler, F. and F. T. Frerichs (1848). "Concerning the modifications which particular organic materials undergo in their transition to the urine." Ann Chem Pharm 63: 335.

Xing, L. L., Hongling; Giesy, John; Zhang, Xiaowei; Yu, Hongxia (2012). "Probabilistic ecological risk assessment for three chlorophenols in surface waters of China." Journal of Environmental Sciences 24(2): 329-334. 
Zhang, X., et al. (2010). "Tissue-specific in vivo bioconcentration of pharmaceuticals in rainbow trout (Oncorhynchus mykiss) using space-resolved solid-phase microextraction." Environ Sci Technol 44(9): 3417-22.

Zhou, S. N., et al. (2008). "Application of solid-phase microextraction for in vivo laboratory and field sampling of pharmaceuticals in fish." Environ Sci Technol 42(16): 6073-9.

Zuccato, E., et al. (2000). "Presence of therapeutic drugs in the environment." Lancet 355(9217): 1789-90. 
VITA

JIAN WANG

1999-2003

B.Sc, Microbiology and Biochemical Pharmaceutics

Jilin University, China

2003-2005

M.Sc, Pharmaceutical Analysis

Jilin University, China

2005-2007

Research Laboratory Technician, Research Center

for Drug Metabolism, Jilin University, China

\section{PUBLICATIONS AND PRESENTATIONS}

Jian Wang, Piero Gardinali, Analysis of selected pharmaceuticals in fish and the fresh water bodies directly affected by reclaimed water using liquid chromatography-tandem mass spectrometry, Analytical and Bioanalytical Chemistry (2012) 404:2711-2720

Jian Wang, Piero Gardinali, Uptake and depuration of pharmaceuticals in reclaimed water by mosquito fish (Gambusia holbrooki): A worst case multiple exposure scenario, Environmental Toxicology and Chemistry (2013), in press

Jian Wang, Piero Gardinali, Identification of phase II pharmaceutical metabolites in reclaimed water using high resolution benchtop Orbitrap mass spectrometry, manuscript ready for submission to Analytical Chemistry, (2013)

Natalia Quinete, Jian Wang, Adolfo Fernandez, Joffre Castro and Piero Gardinali, Outcompeting GC for the detection of legacy chlorinated pesticides: online-SPE UPLC APCI/MSMS detection of endosulfans at part per trillion levels, Analytical and Bioanalytical Chemistry (2013), in press

Jian Wang, Piero Gardinali, Uptake and depuration of the pharmaceuticals in reclaimed water by mosquito fish (Gambusia holbrooki), SETAC North America 33rd Annual Meeting, Nov 15th, Long Beach, CA.

Jian Wang, Improving sample throughput and reducing carryover by reprogramming the CTC autosampler for on-line LC-MS/MS analysis using 
EQUAN system, Thermo Scientific Productivity Seminar Series on March 27th 2012, FortLauderdale FL

Jian Wang, Cesar Ramirez, Piero Gardinali, Simultaneously determination the anionic surfactant DOSS and its degradates in multimedia samples by liquid chromatography tandem mass spectrometry, The 59th ASMS Conference on June 6th, 2011, Denver CO.

Jian Wang, Piero Gardinali, Analysis of selected pharmaceuticals in biological tissues and study of occurrence and fate of these pharmaceuticals in aquatic environment directly impacted by reclaimed water irrigation, SETAC Europe 21st Annual Meeting, May 15th 2011, Milan, Italy.

Jian Wang, Piero Gardinali, Improving sample throughput by tweaking the CTC autosampler program for on-line LC-MS/MS analysis using the EQUAN system, The 7th LC-MS/MS workshop on June 13-14th, 2011, Buffalo NY.

Jian Wang, Piero Gardinali, Analysis of selected pharmaceuticals and personal care products in fish tissues, SETAC North America 31st Annual Meeting, November 9th, 2010, Portland OR.

Jian Wang, Piero Gardinali, Analysis of selected pharmaceuticals and personal care products in biological tissues, SETAC Asia Pacific 2010 Annual Meeting, June 4-7, Guangzhou China. 